\title{
Efficacy of an indicated prevention strategy for long-term sickness absence from an economic, health and stakeholders' perspective
}

Citation for published version (APA):

Klasen, S. H. (2022). Efficacy of an indicated prevention strategy for long-term sickness absence from an economic, health and stakeholders' perspective. [Doctoral Thesis, Maastricht University]. Maastricht University. https://doi.org/10.26481/dis.20220106sk

Document status and date:

Published: 01/01/2022

DOI:

10.26481/dis.20220106sk

Document Version:

Publisher's PDF, also known as Version of record

Please check the document version of this publication:

- A submitted manuscript is the version of the article upon submission and before peer-review. There can be important differences between the submitted version and the official published version of record.

People interested in the research are advised to contact the author for the final version of the publication, or visit the DOI to the publisher's website.

- The final author version and the galley proof are versions of the publication after peer review.

- The final published version features the final layout of the paper including the volume, issue and page numbers.

Link to publication

\footnotetext{
General rights rights.

- You may freely distribute the URL identifying the publication in the public portal. please follow below link for the End User Agreement:

www.umlib.nl/taverne-license

Take down policy

If you believe that this document breaches copyright please contact us at:

repository@maastrichtuniversity.nl

providing details and we will investigate your claim.
}

Copyright and moral rights for the publications made accessible in the public portal are retained by the authors and/or other copyright owners and it is a condition of accessing publications that users recognise and abide by the legal requirements associated with these

- Users may download and print one copy of any publication from the public portal for the purpose of private study or research.

- You may not further distribute the material or use it for any profit-making activity or commercial gain

If the publication is distributed under the terms of Article $25 f a$ of the Dutch Copyright Act, indicated by the "Taverne" license above, 
Efficacy of an indicated prevention strategy for long-term sickness absence from an economic, health and stakeholders' perspective

Sophie Heloïse Klasen 
ISBN: 978-90-831893-8-3

Typografie en zetwerk: Jos Bruystens, Maastricht Druk: Printservice Ede

\section{(c) S. Klasen 2021}

All rights are reserved. No part of this book may be reproduced, distributed, stored in a retrieval system, or transmitted in any form or by any means, without prior written permission of the author. 


\title{
Efficacy of an indicated prevention strategy for long-term sickness absence from an economic, health and stakeholders' perspective
}

\author{
PROEFSCHRIFT \\ ter verkrijging van de graad van doctor aan de Universiteit Maastricht, \\ op gezag van de Rector Magnificus, Prof. dr. Rianne M. Letschert \\ volgens het besluit van het College van Decanen, \\ in het openbaar te verdedigen op \\ donderdag 6 januari 2022 om 16:00 uur
}

door

Sophie Heloïse Klasen 


\section{Promotor}

Prof. dr. IJ. Kant

\section{Co-promotor}

Dr. L.G.P.M. van Amelsvoort

\section{Beoordelingscommissie}

Prof. dr. F.R.H. Zijlstra (voorzitter)

Prof. dr. A.J. van der Beek (VU Medisch Centrum, Amsterdam)

Prof. dr. U. Bültmann (Rijksuniversiteit Groningen)

Dr. P. Lemmens

Prof. dr. A.T.G. Paulus

This project was funded by the Netherlands Organisation for Health Research and Development (ZonMw grant no. 50-53125-98-027). 


\section{Table of contents}

Chapter 2 Efficacy of an indicated prevention strategy regarding health behaviour-related outcomes; health and well-being, social interactions, and healthcare usage over time

Chapter 3 Economic evaluation of an indicated prevention strategy focused on future long-term sickness absence

Chapter 4 Efficacy of an indicated prevention strategy on sickness absence and termination of the employment contract: a 5-year follow-up study

Chapter 5 The impact of an indicated prevention strategy on future sickness absence spells according to their diagnostic labels and attributions

Chapter 6 Exploring expected and perceived facilitators and barriers of an indicated prevention strategy to prevent future long-term sickness absence. A qualitative study among employers and employees

Chapter 7 General discussion

Chapter 8 Impact

Addendum Summary

Nederlandse samenvatting

Dankwoord

About the author 

Chapter 1

\section{General introduction}

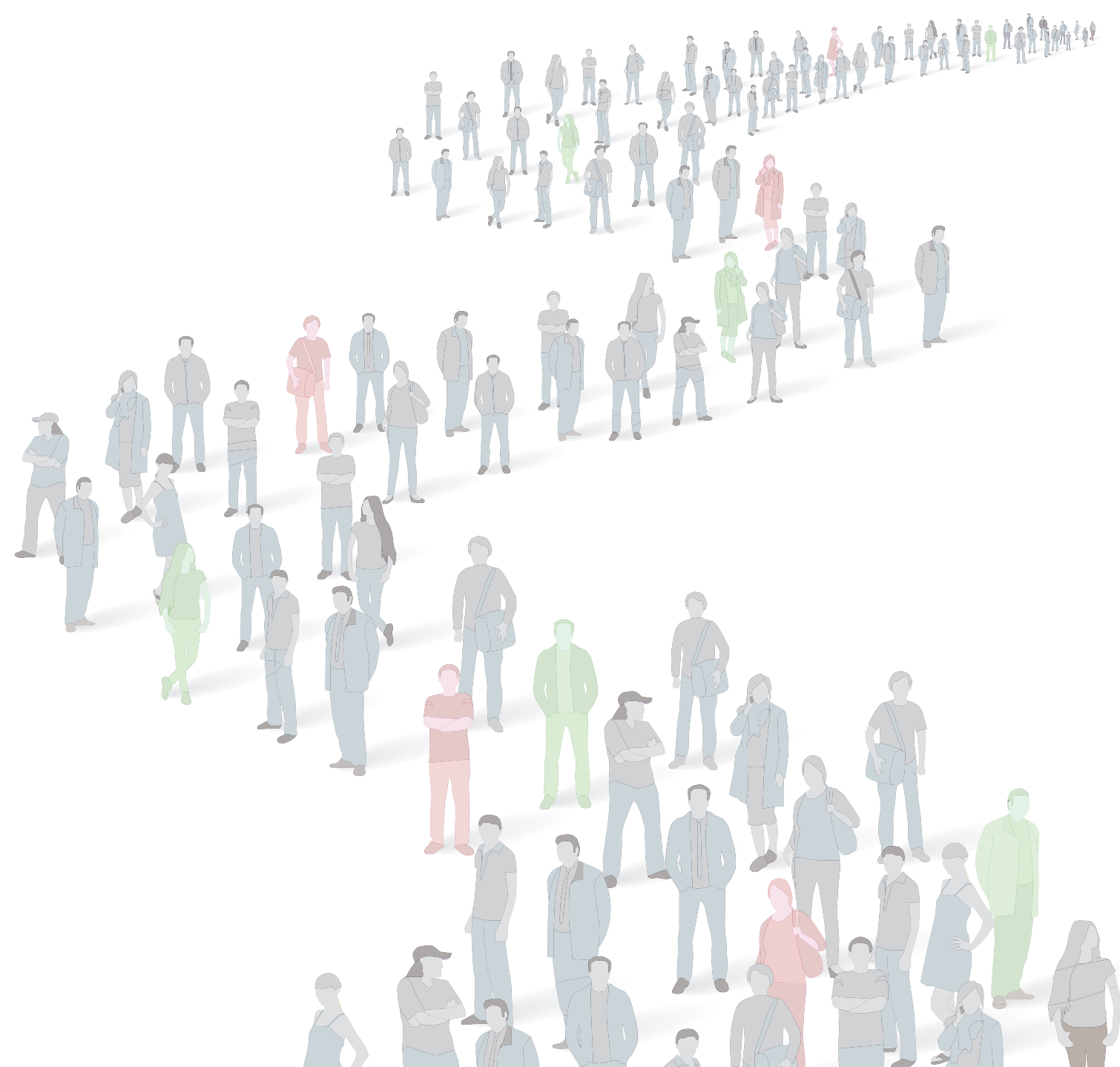





\section{Long-term sickness absence}

Sickness absence (SA), remains a large burden for developed countries and is related with high societal and personal costs [1-4]. Several countries, such as the Netherlands, are at particularly high risk as the total SA rate in the Netherlands was 4.9\% in 2020 [5], which is higher than the European average [6]. This results in an estimated yearly $€ 13.3$ billion in cost for Dutch employers, of which $€ 6.1$ billion is associated with work-related SA and $€ 7.1$ billion with non-work-related SA [4]. The frequency of short periods of SA is much higher than the frequency of long periods of SA. However, the less frequent long periods of SA are responsible for most of the total days of SA [4]. This is illustrated by the finding that all SA periods of 28 or more consecutive days, referred to as long-term sickness absence or LTSA, is responsible for $75 \%$ of the costs related to SA [7]. Furthermore, LTSA often has a negative effect on the health of employees, which could lead to permanent work disability and early retirement, and may over time create financial difficulties for the workers involved [1-3, 8-10]. Overall, LTSA is associated with high costs for the employee, the employer, and society $[1-3,11,12]$. Employers and society are especially confronted with the costs of sick leave in terms of benefits and disability pensions [13]. Since LTSA puts a large burden on society, the employees, and employers, the prevention of LTSA can be key in reducing its negative impact.

\section{Prevention of LTSA}

Prevention can be distinguished into three main groups: primary, secondary, and tertiary prevention [14]. Primary prevention focuses on preventing health complaints in a healthy population by limiting and/or reducing risk factors related to ill health. Secondary prevention is focused on early recognition and subsequent early intervening for individuals, to early recognize symptoms of the disease/condition to prevent the progression of symptoms. Tertiary prevention is focused on the reduction of the negative impact of a disease/condition by reducing disease-related complications [14].

Another prevention classification system, with significant overlap with the before mentioned division, was developed by Gordon, which distinguished universal, selective, indicated, and healthcare-related prevention [15]. Universal prevention is focused on a healthy population to prevent the onset of a disease/condition. Selective prevention fixates on a population exposed to a specific risk factor for a certain disease/condition. Furthermore, indicated prevention addresses individuals with early symptoms of a disease/condition which do not meet the full criteria of a disease/condition and/or are at high risk to develop a specific disease/condition. These individuals are in an early stage of their disease/condition. Lastly, healthcare-related prevention aims at improving the outcome of individuals with a specific disease/condition [16].

Multiple strategies have been aimed at reducing or preventing LTSA, building on the 
principles of general, selective, individual (indicated) prevention or improvements in the return to work process [17-19]. Longer periods of SA are associated with a reduced probability of returning to work $[7,20]$. The period away from work could lead to a detachment between the workplace and the worker, possibly reducing the efficacy of return to work interventions [7, 21].

Many different factors may determine the aetiology of LTSA such as gender, age, poor health, lifestyle, physical workload, psychosocial working conditions, legislation, collective labour agreements, corporate culture, and previous history of SA [22-25]. Its aetiology is therefore considered to be multifactorial, resulting in many interrelated explanatory factors $[3,26]$. And as such, a preventive approach based on individual (indicated) prevention might therefore be assumed to result in better outcomes. This preventive approach is not restricted to one or two explanatory factors but can address a broad range of potentially interrelated factors $[26,27]$. This in contrast to population or general prevention where interventions are often restricted to one or two risk factors. For an indicated prevention approach to prevent future LTSA, it is imperative to treat individuals who experience incipient complaints and are therefore at high risk of LTSA in the future but are not yet on sick leave.

A recent meta-analysis [28] showed the efficacy of multiple indicated prevention strategies regarding the decrease in days of SA. One of these strategies will be the central focus of this thesis.

\section{Elaboration of an indicated prevention strategy to prevent future LTSA}

Researchers of Maastricht University, in close collaboration with the occupational health service 'Beter', developed an indicated prevention strategy (IPS) to prevent future LTSA. This IPS focuses on individuals at high risk for future LTSA, which can be seen as secondary prevention since they already have symptoms related to LTSA. This IPS can also be classified as an indicated prevention strategy (IPS), since it is focused on individuals with early symptoms, indicative of a high risk for LTSA.

The IPS consists of three steps, which address the prediction of LTSA and the early treatment to prevent LTSA. Figure 1 gives a graphic overview of the steps involved in this IPS. 
(1) Screening questionnaire

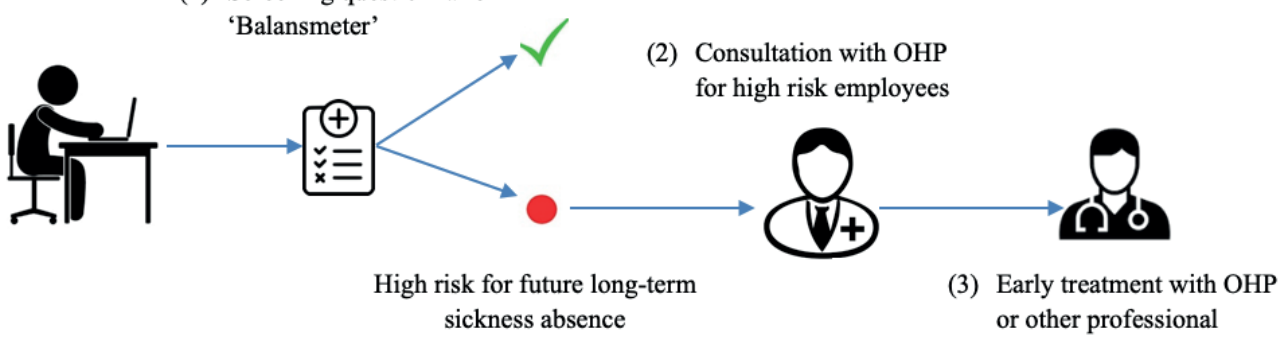

Figure 1: Indicated prevention strategy; prediction and early consultation to prevent future LTSA

During the first step, the employees receive a screening questionnaire in Dutch called the 'Balansmeter', which includes 34 closed-ended questions predictive for future sick leave. The questions are very diverse, relating to the characteristics of the participant's personal situation, working conditions, psychological job demand, health status (including mental), SA history, and demographic factors. A total score is based on an algorithm that considers all predictive factors [29]. A pre-defined cut-off point is used to identify employees at high risk for future LTSA. The screening data is protected by the doctorpatient relationship and is part of the employee's medical file and will therefore never be shared with the employer, except with the employee's explicit consent. Different versions have been developed for office and industry workers and male and female workers [30]. The Balansmeter is internally validated on data from the Maastricht Cohort study and externally validated in a large sample of employees [29, 31].

After the screening step, the employees identified as being at high risk for future LTSA receive an invitation from the occupational physician or occupational health professional for an extensive, one-to-one consultation (step 2). During this structured early consultation, the results of the screening questionnaire are discussed, and a broad range of additional anamneses might be performed to evaluate the options for treatment or guidance. $\mathrm{A}$ special training is made available for the occupational physicians and occupational health professionals to perform a structured early consultation, but attendance is not obligatory [32]. The last step consists of a preventive intervention. This targeted intervention is directly focused on the identified issue. Many different interventions are applied, e.g. lifestyle interventions, psychological interventions, interventions by company counsellors. A detailed overview of the preventive intervention is available in Kant et al. 2008 [30]. The IPS as described above is implemented in several companies with a current screening frequency of once every three years. This frequency has not yet been validated, but it is similar to the frequency of other occupational prevention interventions.

The efficacy of this IPS was assessed in a randomized controlled trial (RCT). The RCT, 
further referred to as RCT I, started in 2003 with $n=9,863$ employees, of whom $n=4,950$ responded to the questionnaire [30]. Employees were eligible for inclusion when they scored above a pre-defined cut-off point, which determined their classification of being at high risk for future LTSA. The exclusion criteria were being fully or partly absent from work, employees who left the company during the RCT period, pregnant employees, and employees who were receiving care from the occupational physician at the time of completing the questionnaire [30]. This resulted in n=263 employees at high risk for LTSA who were randomized in an experimental group receiving an early consultation from the occupational physician, or in a control group receiving care as usual. This resulted in a statistical significantly lower average sick leave duration in the intervention group (mean 18.98; SD 29.50) compared to the control group (mean 31.13; SD 55.47) over a total followup period of one year ( $p=0.007)$ [30].

It was hypothesized that the IPS might be even more effective for a specific target group, for which a targeted intervention was applied. This was studied in a separate RCT, further referred to as RCT II. RCT II was focused on employees at high risk for future LTSA and with relatively mild depressive complaints. The selection of study participants for this RCT, started in 2007 with $n=23,973$ employees, of whom $n=9,157$ responded to the questionnaire [19]. Employees were eligible for inclusion when they were classified as being at high risk for future LTSA, as indicated by the 'Balansmeter' and additionally experienced mild depressive complaints. The exclusion criteria were being fully or partly absent from work, employees who left the company during the RCT period, pregnant employees, employees who were receiving care from the OP at the time of completing the questionnaire, and employees who were receiving care from the psychologist/ psychotherapist during the time of completing the questionnaire. This resulted in $n=139$ employees eligible for randomization [19].

Participants in the intervention group were invited for a psychological treatment based on Problem Solving Therapy (PST) and Cognitive Behavioural Therapy (CBT). This preventive intervention was specially developed to enhance the coping ability of employees and to improve their well-being. A more detailed overview of this preventive intervention is available in Lexis et al. 2011 [19]. Also in this RCT, a statistically significant difference was found in total SA duration between the intervention group (mean 27.5; SD 44.7) and control group (mean 50.8; SD 75.7) over 12 months of follow-up ( $p=0.017$ ). A more detailed overview of this preventive intervention is available in Lexis et al. 2011 [19].

The studies described in this thesis are follow-up studies of these original RCTs with a prolonged follow-up and additional data collection. 
However, despite these promising results, the implementation of the IPS is lagging and therefore more research is necessary to substantiate the potential business case of such an approach build on the principles of indicated prevention [3]. In the following paragraphs, the necessary elements for the substantiating of the IPS are described.

\section{IPS impact beyond sickness absence}

Musculoskeletal disorders and stress-related ill health or psychological issues are known for their association with LTSA. However many other different factors have been identified, which may be involved in why a person reports sick from work or not [33, 34]. Feelings of ill health or a disease classification by a doctor can lead to reporting sick from work [35-37]. On the other hand, a person may not report sick from work if he/she feels their illness is not severe enough, or if no benefits are seen from a diagnosis/treatment [37-39]. All this illustrates that other reasons besides ill health can affect the choice of reporting sick [40]. Therefore, the choice to report sick can thus also be seen as a health behaviour because there is often a choice in reporting sick or not (despite not always perceived by the employee as an explicit choice).

Due to the focus of this IPS on the reduction of future LTSA, a substantial part of this intervention may best be perceived as having a focus on changing health behaviour. Therefore, this IPS is preferably understood and explained by the Health Belief Model (HBM), which is widely used to predict health-related behaviour [41]. The HBM focuses on individual differences in beliefs and attitudes, as the personally perceived threat of a certain disease or condition is assumed to be strongly associated with health behaviour [41]. As multiple aspects of this IPS influence the perceived threat of a disease/condition by the employees, it might influence their health behaviour. Since this IPS showed a large positive change in health behaviour in terms of SA, it might similarly affect other outcomes related to health behaviour.

Multiple studies have examined the effects on health behaviour-related outcomes, e.g. harmful behaviour (a.o. smoking), protective behaviour (a.o. exercise), healthcare resources (a.o. medication intake), and health (a.o. self-perceived health) [42-47]. Furthermore, health behaviour also showed to be affected by the environment and vice versa $[48,49]$. Social support showed to be associated with health behaviour, and a recent study even indicated that health behaviour might also affect social support [50, 51]. However, to our knowledge, no earlier studies have been conducted on the efficacy of an IPS targeting the prevention of future LTSA with a focus on health behaviour-related outcomes. It is assumed this information will generate a broader overview of the efficacy of this IPS and generate important knowledge for improving implementation. 


\section{How to obtain detailed insight into costs and effects}

The efficacy of this IPS has been assessed in terms of days of SA and health behaviourrelated outcomes. However, to enable the assessment of the effects and costs and ensure a fair comparison with other preventive interventions, an economic evaluation is perceived as indispensable. This comparison is most relevant for companies and society since it enables the choice to be made between different effective interventions, based on the greatest effects for the lowest costs. An economically advantageous outcome might also encourage broader implementation of this strategy.

A cost-effectiveness analysis (CEA) is seen as an appropriate economic evaluation method, as it determines the incremental cost-effectiveness ratio (ICER), which indicates the additional investment needed for this IPS to gain one extra unit of health behaviourrelated outcome (e.g. self-perceived health) compared to the care as usual.

A recent meta-analysis showed that the cost-effectiveness has been reported for only one other indicated prevention intervention similar to the one described above $[11,28]$. Therefore, the evidence of cost-effective interventions focused on indicated prevention of future LTSA remains uncertain. Furthermore, it is of added value to perform a CEA as seen from multiple perspectives (e.g. societal, healthcare and employer) to understand the different and sometimes opposing views of stakeholders regarding this strategy. Overall, this economic evaluation may give more insight into the facilitators and barriers for the stakeholders involved in this strategy.

\section{Long-term efficacy IPS?}

The somewhat slow and cumbersome implementation process may partially be explained by the lack of interest from employers, since they may not yet be convinced about the cost-effectiveness or efficacy of this strategy. The screening uptake and participation in the early intervention were only moderate in companies where the IPS has been implemented, suggesting that there may be hindering factors among the employees as well [52]. Investigating the long-term efficacy of this IPS might be a way to establish and illustrate potential long-term effects and as such, might help in underpinning the relevance of an indicated preventive approach for addressing LTSA. Furthermore, it might be that differences in interests between employers and employees, such as financial interests, may further hamper the implementation. For example, many employers are often more focused on cost reduction, whereas employees are often more focused on the possible improvement in personal health and workability $[53,54]$. These discrepancies may inhibit the adoption and implementation of this strategy.

The IPS has already shown to significantly reduce SA during the first year after the intervention $[30,55]$. The substantial reduction in days of SA during the first year may be accompanied by help-seeking behaviour which could extend the one-year efficacy 
$[52,56,57]$. In particular, the early contact with the occupational physician during RCT I was assumed to be very important, since it may result in sustainable work adjustments or other improvements in the work situation. A recent meta-analysis [28] showed that the long-term effects have not yet been researched for indicated prevention strategies in an occupational health setting. Moreover, this meta-analysis showed that the long-term effects of RCT II type interventions, based on Problem Solving Therapy (PST) or Cognitive Behavioural Therapy (CBT), did not report long-lasting results [28]. However, CBT was associated with long-term results in the reduction of depressive complaints that extended far beyond the end of treatment [58-63]. This could suggest that the intervention from RCT II might also lead to a sustainable decrease in SA long after the intervention has ended.

\section{Is the IPS efficacy differential?}

In earlier studies, the overall efficacy of this IPS was calculated for the total group. However, the efficacy of this strategy may be different for subgroups of employees with different spectra of factors and or complaints underlying the high risk for future LTSA at the time of intervention.

According to a recent national Dutch report, SA was often related to psychological complaints ( $27 \%$ of the cases), musculoskeletal complaints $(24 \%)$, other attributes (e.g. heart disease, respiratory diseases etc. (37\%) followed by the flu and having a cold (12\%) [4]. Furthermore, SA spells were partly or mainly attributed by work for $47 \%$ of the cases, of which psychosocial determinants were the largest attributes of SA with $27 \%$ [4]. Furthermore, a meta-review by Joyce et al. 2016 indicated that workplace interventions can significantly decrease the burden of common mental disorders, which could indicate that the days of SA associated with mental disorders might be affected as well [64].

Moreover, systematic reviews showed fairly positive results regarding the effects of workplace interventions on musculoskeletal disorders which may indicate that a relatively larger reduction of SA associated with this type of disease classification, might be achieved by interventions, as compared to other types $[65,66]$.

The above-mentioned studies suggest that workplace interventions can decrease the specific types of disease/illness. However, it remains uncertain, whether specific interventions at the workplace can have differential effects on complaints underlying the high risk for future LTSA. Insights into these differential effects may be crucial for further optimizing and tailoring this IPS.

\section{Improving uptake and compliance}

Indications of factors facilitating or hindering implementation related to general, universal, or selective prevention in occupational health prevention have been described. These studies identified relevant potential facilitators and barriers for employers and employees, 
which could also be applicable to indicated prevention strategies. For employers, the most important facilitators that were identified, were a need to improve the productivity and well-being of employees and to reduce costs by decreasing healthcare costs associated with absenteeism and permanent disability $[67,68]$. Potential barriers often mentioned by the employers were the high costs of an intervention, time scarcity of the employees and logistical issues [69]. For employees, the most commonly mentioned facilitators were improvements in their expected general well-being and their physical and psychological health by preventive interventions $[67,70]$. Privacy issues and fear of discrimination/ stigmatization were often mentioned by employees as important barriers for the uptake of interventions [71].

The facilitators and barriers of an IPS focused on the reduction of future LTSA have only been studied for the group of occupational physicians directly involved in the early intervention, but not yet for other relevant stakeholders, namely the employers and employees. De Brouwer et al. 2017 showed that the most important barriers for these occupational physicians were appropriate training, communication skills, and privacy issues related to the labelling of employees with a high-risk status [32]. Due to the early character of this IPS, before employees report sick from work, and its primary focus on high-risk employees for future LTSA, it may result in different facilitators and barriers than described in the above mentioned studies. A comparable study indicated that questions concerning the risk perception of a disease may create misconceptions, while employees may be unaware of the true meaning of being at high risk [72]. Investigating the facilitators and barriers of this IPS for employers and employees is very important since insight in these factors is crucial for enhancing the implementation.

\section{General aim}

Considering the relevance of preventing LTSA for the employees, employers, and society, a thorough evaluation of an indicated prevention strategy for the prevention of future LTSA is desirable. This can generate important knowledge, which could benefit a broader implementation of this strategy in the future, and as such contribute to a reduction of LTSA and related outcomes. Therefore, this thesis aims to examine the IPS efficacy regarding sickness absence and health behaviour-related outcomes (health and well-being, social interactions, and healthcare usage) to enable a solid economic evaluation. A second aim is to evaluate the long-term efficacy of the IPS regarding sickness absence as well as the efficacy regarding ill health (diagnostic labels and attributions). Furthermore, this thesis aims to investigate the facilitators and barriers for employers and employees regarding this IPS, which could benefit or hinder future implementation. 


\section{Rationale, aims, and general approach}

The general aim includes five main research questions:

1. What is the efficacy of this IPS focused on the reduction of future long-term sickness absence in terms of health behaviour-related outcomes during 12 months of followup?

2. What is the cost-effectiveness of this IPS seen from a societal, healthcare, and employer perspective during 12 months of follow-up?

3. What is the efficacy during 5 years of follow-up of an IPS focused on the reduction of future long-term sickness absence in terms of sickness absence parameters and termination of employment?

4. What is the impact of an IPS focused on the reduction of future long-term sickness absence regarding sickness absence spells according to their diagnostic labels and attributions?

5. What are the expected and perceived facilitators and barriers regarding this IPS for employers and employees?

The first four research questions are part of a prolonged follow-up study with additional data collected from the participants from RCT I and additionally from RCT II participants for research question three. The last research question was based on a qualitative study, with semi-structured interviews which were analysed thematically.

\section{Outline of the thesis}

In chapter 2 of this thesis, the focus is on the general high-risk population for LTSA and the efficacy of the indicated prevention strategy on health and well-being, social interactions, and healthcare usage over time. Chapter 3 shows the economic evaluation of an indicated prevention strategy from a societal, healthcare, and employer perspective. In chapter 4, the results of a prolonged follow-up study from RCT I are presented regarding the longterm efficacy of an indicated prevention strategy on sickness absence parameters and termination of the employment contract. Chapter 5 presents the results regarding the impact of this IPS on sickness absence according to their diagnostic labels and attributions. Chapter 6 explores the expected and perceived facilitators and barriers of an indicated prevention strategy to prevent future LTSA for employers and employees. At last, Chapter 7 provides a general discussion, presenting an overview of the results of this thesis, its strengths and weaknesses, implications for stakeholders, and implications for future studies. In Figure 2, an overview of the data included in this thesis is presented. 
Figure 2: Overview of the data included in this thesis

\begin{tabular}{|c|c|c|c|}
\hline & RCT I & RCT II & Interviews \\
\hline Chapter 2 & $\begin{array}{l}\text { Health and well-being, social } \\
\text { interactions, and healthcare } \\
\text { usage during } 6 \text { and } 12 \text { months } \\
\text { of follow-up }\end{array}$ & n.a. & n.a. \\
\hline Chapter 3 & $\begin{array}{l}\text { Economic evaluation as seen } \\
\text { from a societal, healthcare, } \\
\text { and employer perspective }\end{array}$ & n.a. & n.a. \\
\hline Chapter 4 & $\begin{array}{l}5 \text { years follow-up of SA \& } \\
\text { determination of employment }\end{array}$ & $\begin{array}{l}5 \text { years follow-up of SA \& } \\
\text { determination of employment }\end{array}$ & n.a. \\
\hline Chapter 5 & $\begin{array}{l}5 \text { years follow-up of SA } \\
\text { according to diagnostic labels/ } \\
\text { attributions }\end{array}$ & n.a. & n.a. \\
\hline Chapter 6 & n.a. & n.a. & $\begin{array}{l}\text { Interviews with } \\
\text { employers and } \\
\text { employees }\end{array}$ \\
\hline
\end{tabular}




\section{References}

1. Labriola, M., Conceptual framework of sickness absence and return to work, focusing on both the individual and the contextual level. Work, 2008. 30(4): p. 377-387.

2. Lund, T., et al., Using administrative sickness absence data as a marker of future disability pension: the prospective DREAM study of Danish private sector employees. Occupational and Environmental Medicine, 2008. 65(1): p. 28-31.

3. van Amelsvoort, L.G.P.M., N.W.H. Jansen, and I. Kant, Addressing long-term sickness absence: moving beyond disease, illness and work-related factors for effective prevention. Scandinavian Journal of Work Environment \& Health, 2017. 43(1): p. 1-4.

4. Kraan K., I.d.M.M., de Vroome E., Health and safety balans; sickness abence and work incapacity. [In Dutch: Arbobalans 2020; ziekteverzuim en arbeidsongeschiktheid]. 2020. p. 111-127.

5. CBS. Sickness absence highest in fourth term in 18 years. [In Dutch: Ziekteverzuim vierde kwartaal hoogste in 18 jaar]. 2021 [cited 2020 11-11-2020]; Available from: https://www.cbs. nl/nl-nl/nieuws/2021/06/ziekteverzuim-vierde-kwartaal-hoogste-in-18-jaar.

6. Volksgezondheidenzorg. International comparison of sickness absence. [In Dutch: Internationale vergelijking ziekteverzuim]. 2021 [cited 2020 15-11-2020]; Available from: https://www.volksgezondheidenzorg.info/onderwerp/ziekteverzuim/regionaal-internationaal/internationaal\#bronverantwoording.

7. Henderson, M., et al., Work and common psychiatric disorders. Journal of the Royal Society of Medicine, 2011. 104(5): p. 198-207.

8. Helgesson, M., et al., Sickness absence at a young age and later sickness absence, disability pension, death, unemployment and income in native Swedes and immigrants. Eur J Public Health, 2015. 25(4): p. 688-92.

9. Vingård, E., K. Alexanderson, and A. Norlund, Chapter 9. Consequences of being on sick leave. Scandinavian journal of public health, 2004. 32(63_suppl): p. 207-215.

10. Wiencke, M., M. Cacace, and S. Fischer, Healthy at Work: Interdisciplinary Perspectives. 2016: Springer.

11. Taimela, S., et al., An occupational health intervention programme for workers at high risk for sickness absence. Cost effectiveness analysis based on a randomised controlled trial. Occupational and environmental medicine, 2008. 65(4): p. 242-248.

12. Henderson, M., N. Glozier, and K.H. Elliott, Long term sickness absence. 2005, British Medical Journal Publishing Group.

13. Volksgezondheidenzorg. Trend in costs for disability benefits 2002-2019. [In Dutch: Trend in kosten arbeidsongeschiktheidsuitkeringen]. . 2021 12-02-2021]; Available from: https:// www.volksgezondheidenzorg.info/onderwerp/arbeidsongeschiktheid/kosten/kosten.

14. J.P. Mackenback, P.J.v.d.M., Public health care and health care. [In dutch: Volkgezondheid en gezondheidszorg]. Vol. 4de druk. 2009: Elsevier. 
15. Gordon Jr, R.S., An operational classification of disease prevention. Public health reports, 1983. 98(2): p. 107.

16. CVZ, Of prevention assured. [In Dutch: Van preventie verzekerd]. 2007. p. 17-18.

17. Vogel, N., et al., Return-to-work coordination programmes for improving return to work in workers on sick leave. Cochrane Database of Systematic Reviews, 2017(3).

18. Notenbomer, A., et al., Effect of an eHealth Intervention to Reduce Sickness Absence Frequency Among Employees With Frequent Sickness Absence: Randomized Controlled Trial. Journal of medical Internet research, 2018. 20(10): p. e10821.

19. Lexis, M.A., et al., Prevention of long-term sickness absence and major depression in highrisk employees: a randomised controlled trial. Occupational and environmental medicine, 2011. 68(6): p. 400-407.

20. Kivimaki, M., et al., Sickness absence as a risk marker of future disability pension: the 10town study. J Epidemiol Community Health, 2004. 58(8): p. 710-1.

21. Franche, R.-L., et al., Workplace-based return-to-work interventions: a systematic review of the quantitative literature. Journal of occupational rehabilitation, 2005. 15(4): p. 607-631.

22. Beemsterboer, W., et al., A literature review on sick leave determinants (1984-2004). International journal of occupational medicine and environmental health, 2009. 22(2): p. 169-179.

23. Vingård, E., et al., Long-term sick-listing among women in the public sector and its associations with age, social situation, lifestyle, and work factors: a three-year follow-up study. Scandinavian journal of public health, 2005. 33(5): p. 370-375.

24. Andersen, L.L., et al., Long-term sickness absence from combined factors related to physical work demands: prospective cohort study. European journal of public health, 2018. 28(5): p. 824-829.

25. Aronsson, V., et al., Can a poor psychosocial work environment and insufficient organizational resources explain the higher risk of ill-health and sickness absence in human service occupations? Evidence from a Swedish national cohort. Scandinavian journal of public health, 2019. 47(3): p. 310-317.

26. Schultz, I.Z. and R.J. Gatchel, Handbook of return to work: From research to practice. Vol. 1. 2015: Springer.

27. Rose, G., Sick individuals and sick populations. International journal of epidemiology, 2001. 30(3): p. 427-432.

28. Salomonsson, S., E. Hedman-Lagerlöf, and L.-G. Öst, Sickness absence: a systematic review and meta-analysis of psychological treatments for individuals on sick leave due to common mental disorders. Psychological medicine, 2018. 48(12): p. 1954-1965.

29. Kant, I., et al., Screening questionnaire Balansmeter proved successful in predicting future long-term sickness absence in office workers. Journal of clinical epidemiology, 2009. 62(4): p. 408-414. e2. 
30. Kant, I., et al., Structured early consultation with the occupational physician reduces sickness absence among office workers at high risk for long-term sickness absence: A randomized controlled trial. Journal of Occupational Rehabilitation, 2008. 18(1): p. 79-86.

31. Duijts, S.F., et al., Prediction of sickness absence: development of a screening instrument. Occupational and environmental medicine, 2006. 63(8): p. 564-569.

32. de Brouwer, C.P.M., et al., Experiences of occupational physicians with the implementation of indicated prevention for long term sickness absence. Work-a Journal of Prevention Assessment \& Rehabilitation, 2017. 57(2): p. 157-172.

33. Waddell, G. and A.K. Burton, Concepts of rehabilitation for the management of low back pain. Best Pract Res Clin Rheumatol, 2005. 19(4): p. 655-70.

34. Munir, F., J. Yarker, and C. Haslam, Sickness absence management: encouraging attendance or 'risk-taking'presenteeism in employees with chronic illness? Disability and Rehabilitation, 2008. 30(19): p. 1461-1472.

35. Marmot, M., et al., Sickness absence as a measure of health status and functioning: from the UK Whitehall II study. Journal of Epidemiology \& Community Health, 1995. 49(2): p. 124-130.

36. Kivimäki, M., et al., Sickness absence as a global measure of health: evidence from mortality in the Whitehall II prospective cohort study. Bmj, 2003. 327(7411): p. 364.

37. Wikman, A., S. Marklund, and K. Alexanderson, Illness, disease, and sickness absence: an empirical test of differences between concepts of ill health. Journal of Epidemiology \& Community Health, 2005. 59(6): p. 450-454.

38. Aronsson, G., K. Gustafsson, and C. Mellner, Sickness presence, sickness absence, and selfreported health and symptoms. International Journal of Workplace Health Management, 2011.

39. Aronsson, G. and V. Blom, Work conditions for workers with good long-term health. International Journal of Workplace Health Management, 2010.

40. Johansson, G. and I. Lundberg, Adjustment latitude and attendance requirements as determinants of sickness absence or attendance. Empirical tests of the illness flexibility model. Social science \& medicine, 2004. 58(10): p. 1857-1868.

41. Rosenstock, I.M., Historical origins of the health belief model. Health education monographs, 1974. 2(4): p. 328-335.

42. Michie, S., et al., Evaluating the effectiveness of behavior change techniques in health-related behavior: a scoping review of methods used. Translational behavioral medicine, 2018. 8(2): p. 212-224.

43. Ashton, L.M., et al., Effectiveness of interventions and behaviour change techniques for improving dietary intake in young adults: a systematic review and meta-analysis of RCTs. Nutrients, 2019. 11(4): p. 825.

44. Lancaster, T. and L.F. Stead, Individual behavioural counselling for smoking cessation. Cochrane database of systematic reviews, 2017(3). 
45. Samdal, G.B., et al., Effective behaviour change techniques for physical activity and healthy eating in overweight and obese adults; systematic review and meta-regression analyses. International Journal of Behavioral Nutrition and Physical Activity, 2017. 14(1): p. 42.

46. Craig, B.A., et al., The association between self-rated health and social environments, health behaviors and health outcomes: a structural equation analysis. BMC Public Health, 2018. 18(1): p. 440.

47. Riediger, N.D., A.E. Bombak, and A.N. Mudryj, Health-related behaviours and their relationship with self-rated health among Canadian adults. BMC public health, 2019. 19(1): p. 960.

48. Glanz, K., and Rimer, B. K. , Theory at a Glance: A Guide to Health Promotion Practice. National Cancer Institute, 1995.

49. Stokols, D., et al., Increasing the health promotive capacity of human environments. American journal of health promotion, 2003. 18(1): p. 4-13.

50. Glanz, K., B.K. Rimer, and K. Viswanath, Health behavior and health education: theory, research, and practice. 2008: John Wiley \& Sons.

51. Nelissen, P.T.J.H., Hulsheger, U.R., van Ruitenbeek, G.M.C. \& Zijlstra, F.R. H. , With a little help from my team: Well-being of people with disabilities at work (submitted).

52. Lexis, M.A., et al., Experience of health complaints and help seeking behavior in employees screened for depressive complaints and risk of future sickness absence. Journal of occupational rehabilitation, 2010. 20(4): p. 537-546.

53. Auvinen, A.-M., K. Kohtamäki, and M. Ilvesmäki, Antti, Workplace health promotion and stakeholder positions: a Finnish case study. Archives of environmental \& occupational health, 2012. 67(3): p. 177-184.

54. Downey, A.M. and D.J. Sharp, Why do managers allocate resources to workplace health promotion programmes in countries with national health coverage? Health Promotion International, 2007. 22(2): p. 102-111.

55. Lexis, M.A.S., et al., Prevention of long-term sickness absence and major depression in highrisk employees: a randomised controlled trial. Occupational and Environmental Medicine, 2011. 68(6): p. 400-407.

56. Steenbeek, R., Workers' opinions on the effect of contact with health care providers on sickness absence duration. Work, 2014. 49(3): p. 495-504.

57. Mortelmans, A.K., et al., Work-related sickness absences and mandatory occupational health surveillance. Occupational medicine, 2008. 58(7): p. 464-467.

58. Driessen, E. and S.D. Hollon, Cognitive behavioral therapy for mood disorders: efficacy, moderators and mediators. Psychiatric Clinics, 2010. 33(3): p. 537-555.

59. Hollon, S.D., Does cognitive therapy have an enduring effect? Cognitive therapy and research, 2003. 27(1): p. 71-75.

60. Fava, G.A., et al., Six-year outcome of cognitive behavior therapy for prevention of recurrent depression. American Journal of Psychiatry, 2004. 161(10): p. 1872-1876. 
61. Bockting, C.L., et al., Long-term effects of preventive cognitive therapy in recurrent depression: a 5.5-year follow-up study. Journal of Clinical Psychiatry, 2009. 16(12): p. 1621.

62. Øyeflaten, I., et al., Functioning, coping and work status three years after participating in an interdisciplinary, occupational rehabilitation program. Scandinavian journal of public health, 2014. 42(5): p. 425-433.

63. Nigatu, Y.T., et al., Indicated Prevention Interventions in the Workplace for Depressive Symptoms: A Systematic Review and Meta-analysis. American journal of preventive medicine, 2019. 56(1): p. e23-e33.

64. Joyce, S., et al., Workplace interventions for common mental disorders: a systematic metareview. Psychological medicine, 2016. 46(4): p. 683-697.

65. Sundstrup, E., et al., A systematic review of workplace interventions to rehabilitate musculoskeletal disorders among employees with physical demanding work. Journal of occupational rehabilitation, 2020: p. 1-25.

66. Pieper, C., S. Schröer, and A.-L. Eilerts, Evidence of workplace interventions-A systematic review of systematic reviews. International journal of environmental research and public health, 2019. 16(19): p. 3553.

67. Envicke, B., Investing in a health workforce: the impact of physical wellness on psychological well-being and the critical implications for worker performance. Acad Health Care Manag J. , 2012. 8;(1/2): p. 21-32.

68. Gandy, W.M., et al., Comparing the contributions of well-being and disease status to employee productivity. Journal of occupational and environmental medicine, 2014. 56(3): p. 252-257.

69. Hannon, P.A., et al., Stakeholder perspectives on workplace health promotion: a qualitative study of midsized employers in low-wage industries. American Journal of Health Promotion, 2012. 27(2): p. 103-110.

70. Kivimaki, M., et al., Sickness absence as a global measure of health: evidence from mortality in the Whitehall II prospective cohort study. British Medical Journal, 2003. 327(7411): p. 364368.

71. Madison, K.M., The risks of using workplace wellness programs to foster a culture of health. Health Affairs, 2016. 35(11): p. 2068-2074.

72. Damman, O.C., A.J. van der Beek, and D.R. Timmermans, Workers' knowledge and beliefs about cardiometabolic health risk. Journal of occupational and environmental medicine, 2014. 56(1): p. 92-100. 



\section{Chapter 2}

\section{Efficacy of an indicated presenton strategy regarding health b ayjour- related outcomes; he (th a d well- being, social inter action, and}

healthcare usage ver ime

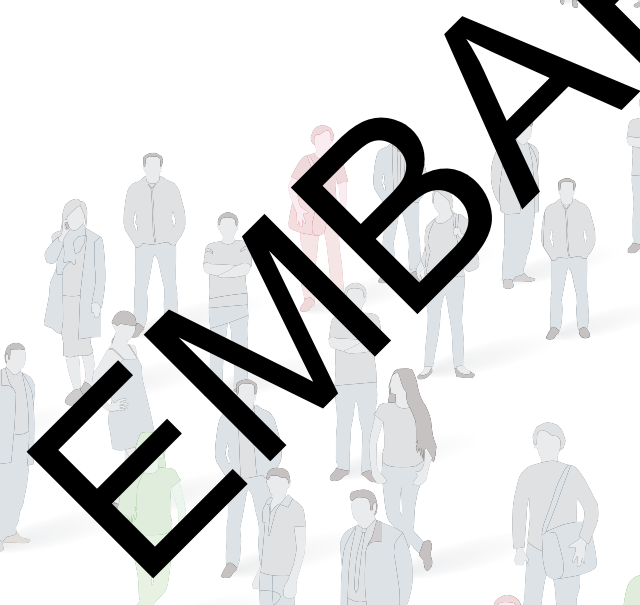

Sophie H Klasen, MSc, Ludovic GPM van Amelsvoort, PhD, Nicole WH Jansen, PhD, Jos JM Slangen, IJmert Kant, PhD

CAPHRI School for Public Health and Primary Care, Department of Epidemiology, Faculty of Health, Medicine and Life Sciences, Maastricht University, Maastricht, The Netherlands. 


\section{Chapter 3}

\section{Economic evaluation of anjidioted prevention strategy focus ov fyture long-term sickness ab enc}

ophie H Klasen, MSc, ${ }^{1}$ Ludovic GPM van Amelsvoort, PhD, ${ }^{1}$ IJmert Kant, PhD, ${ }^{1}$ Nicole WH Jansen, PhD, ${ }^{1}$ Silvia M.A.A. Evers, $\mathrm{PhD}^{2,3}$

${ }^{1}$ CAPHRI School for Public Health and Primary Care, Department of Epidemiology, Faculty of Health, Medicine and Life Sciences, Maastricht University, Maastricht, The Netherlands.

${ }^{2}$ Department of Health Services Research, Maastricht University, the Netherlands. ${ }^{3}$ Trimbos Institute, Netherlands Institute of Mental Health and Addiction Centre of Economic Evaluation \& Machine Learning. 


\section{Chapter 4}

\section{Efficacy of an indicated prevention strategy on sickness absence and termination of the employment contract: a 5-year follow-up study}

Sophie H Klasen, MSc, ${ }^{1}$ Ludovic GPM van Amelsvoort, PhD, ${ }^{1}$ Nicole WH Jansen, PhD, ${ }^{1}$ Jos JM Slangen, ${ }^{1}$ Gladys Tjin A Ton, MD, ${ }^{2}$ IJmert Kant, $\mathrm{PhD}^{1}$

${ }^{1}$ CAPHRI School for Public Health and Primary Care, Department of Epidemiology, Faculty of Health, Medicine and Life Sciences, Maastricht University, Maastricht, The Netherlands

${ }^{2}$ Occupational Health Service Beter, Amsterdam, The Netherlands 


\section{Abstract}

\section{Objective}

It was shown that an indicated prevention strategy (IPS), based on screening and early intervention, can considerably decrease future risk of long-term sickness absence (LTSA>28 days) over one year. Given the nature of the interventions, the potential of an effect extending beyond the original one year of follow-up might be present. This study aims to determine the efficacy of this IPS on LTSA and termination of employment contract over five years by extended follow up of IPS trials.

\section{Methods}

Company records on sickness absence and termination of employment contract over five years were used from two randomized controlled trials (RCT) on the efficacy of the IPS (RCT I employees at high-risk for LTSA: intervention: N=263; RCT II high-risk employees with concurrent mild depressive complaints: intervention: $N=139)$. Survival analysis was used to model time until the first LTSA episode and termination of employment contract.

\section{Results}

RCT I showed a decrease of 43.2 days of sickness absence $(\mathrm{P}=0.05)$ and a lower 5 -year risk of LTSA in the intervention, as compared to the control group [hazard ratio (HR) 0.61 , 95\% confidence interval $(\mathrm{Cl})$ 0.41-0.90], however no considerable impact on employment contract (HR 0.85, 95\% Cl 0.54-1.35) (intention-to-treat, ITT). For RCT II, we found no large difference in days of SA and no difference in LTSA risk over five years (HR 1.31, 95\% CI 0.702.47), whereas the risk of termination of the employment contract was lower (HR 0.62, 95\% Cl 0.39-0.99) (ITT).

\section{Conclusion}

Effects of the IPS were observed over five years, albeit differential between the two approaches. A combination of elements of both interventions might lead to optimal results but needs further study. 


\section{Background}

Long-term sickness absence (LTSA) has large consequences in terms of health and costs for employees, employers, and society [1-3]. LTSA is seen as a precursor of permanent work disability, early retirement due to ill health, and even mortality [3, 4]. Many studies have shown that returning to work after a period of LTSA remains very difficult and may even result in financial difficulties over time due to unemployment $[1,2,5,6]$. Perceived poor health, mental health issues, or chronic conditions are known factors that could determine the termination of the employment contract with the company, as a result of disability pensions or unemployment [7]. Therefore, preventing LTSA may be positively associated with fewer employees having to exit employment due to ill health. In The Netherlands, termination of the employment contract can be related to disability, retirement, job loss, or voluntary leave. Preventing LTSA is of utmost importance and may result in improving the health of employees, fewer costs due to a decrease in days of sickness absence (SA), and the prevention of work disability [3].

Musculoskeletal disorders and stress-related ill health are seen as the most important reasons for LTSA $[8,9]$. However, the etiology of SA is often multifactorial which makes it difficult to comprehend and requires a holistic understanding [10-12]. Many factors have been associated with an increased LTSA risk, for example, age, gender, lifestyle, poor health, SA records, physical workload, and psychosocial working conditions [10, 13-15]. Therefore, individual or indicated prevention might result in better outcomes since it focuses on a broad range of potentially interrelated factors, in contrast to population or general prevention, which is often restricted to one or two factors. Essential is here the focus on treating individuals who are at risk of reporting sick in the future but are not yet currently on sick leave.

Two prerequisites for a successful indicated prevention strategy (IPS) to prevent LTSA are the ability to (i) detect individuals who are at high risk for future LTSA and (ii) provide these individuals with effective treatment at an early stage. A strategy meeting both prerequisites has shown its efficacy in two Dutch randomized controlled trials (RCT) $[16,17]$. While the RCT differed in study population and type of early intervention, both used screening and structured early intervention [16-18]. Earlier studies have shown the efficacy of this prevention strategy in reducing days of SA over a 12-month interval [16, 17]. Furthermore, a recent meta-analysis [19] showed that other interventions based on the principles of IPS could have considerable effects on SA. Duijts et al [20] reported 15.5 compared to 18.8 SA days hazard ratio (HR) of $-0.15,95 \% \mathrm{Cl}(-0.23--0.07)$, Lerner et al [21] showed $7.1 \%$ improvement in productivity due to less $\mathrm{SA}(\mathrm{P}<0.01)$ and 29.5 compared to 26.0 effective weekly hours $(\mathrm{P}=0.008)$ and, over a period of one year, Taimela et al [22] showed a mean difference of 11 days between intervention and control group in favor of 
the intervention group [20-22]. The results from comparable IPS only showed short-term effects (4-24 months), all of which were comparable to the average one-year results for RCT I=12.1 days and RCT II=23.3 days.

However, the long-term efficacy of an IPS has not yet been studied in terms of SA. While the efficacy in terms of decreasing SA during one year of follow-up was large in RCT I, this could indicate that the intervention has lasting effects on help seeking behavior, which could possibly decrease SA over a long time period [23-25]. Especially since one might assume that early contact with the occupational physician (OP) could result in sustainable work adjustments or other improvements in working conditions. With regards to the preventive intervention used in RCT II, which was based on Problem Solving Therapy (PST) and Cognitive behavioral Therapy (CBT), it was found that, over one year, SA as well as depressive complaints decreased [17]. However, given the aim of the intervention (ie, to enhance coping ability), long-lasting effects beyond the reported one year might also be expected for this intervention. Nonetheless, so far, long-lasting effects have not yet been described for this or similar interventions, as apparent from a recent meta-analysis [19]. The expectation of long-lasting effects comes from studies on the effect of CBT in terms of depressive complaints, which suggests that CBT might have enduring effects that extend beyond the end of treatment, supporting our hypothesis that the intervention from RCT II could also lead to a sustainable decrease in SA at 5-years follow-up [26-31]. Demonstrated long-term efficacy is highly relevant for social and economic reasons since LTSA often is associated with high costs [2]. Therefore, this study aimed to examine the efficacy of an indicated prevention strategy to prevent LTSA, through record linkage of the RCTs participant's data on SA parameters and termination of employment over a 5-year follow-up period.

\section{Methods}

\section{Design, procedure and participants}

Two RCT were conducted among office workers who were classified as high risk for future LTSA by a screening questionnaire called the 'Balansmeter' in Dutch. The current paper describes a follow-up study on indicators of labor participation with a focus on SA parameters and termination of the employment contract. Similarities can be found between the preventive interventions in RCT I and RCT II in the timing of the preventive intervention and the use of a screening instrument to classify employees as high risk for LTSA. However, the preventive interventions differ in the type and intensity of treatment. 


\section{Screening instrument}

The screening instrument (Balansmeter) was developed to identify employees at high risk for future LTSA in an office environment before they report sick. The Balansmeter was internally validated on data of the Maastricht Cohort study and externally validated on a large sample of employees from the same company in which both RCT were conducted $[18,32]$. Detailed information about the screening instrument can be found in the supplementary material.

\section{RCT I}

Starting in 2003, RCT I invited 9863 employees to participate in the study, of which 4950 responded to the questionnaire. Employees were selected if they scored above the cutoff point of the Balansmeter, which indicated that they were at high risk for future LTSA. Exclusion criteria were employees (i) already on sick leave, (ii) receiving OP care at the time of completing the screening questionnaire, (iii) who left the company during the $\mathrm{RCT}$ period, and (iv) who were pregnant. This resulted in $\mathrm{N}=263$ employees eligible for allocation in the intervention or control groups. A detailed description of the selection procedure of participants is described elsewhere [16]. The original follow-up period for RCT I was one year, extended to five for the current study. The allocation of participants in RCT I is shown in the online appendix flow diagram S1. The number of study participants decreased over time due to the departure of employees from the company because of disability, retirement, job loss, or voluntary leave.

For RCT I, employees in the intervention group received a structured early consultation by the OP/OHP, which may already be viewed as a short intervention due to the time involvement, often followed by further consultations within the occupational health service. The consultation was held according to a protocol consisting of different steps, in which the main symptoms were discussed and the relation between their symptoms and the risk for future LTSA explained. Finally, the expectations and benefits of early treatment were discussed with the employee. The consultation could then be followed by a targeted intervention to focus directly on the identified issues. Different interventions were applied (eg, psychological interventions, lifestyle interventions, and interventions by company counselors). This resulted in 84 employees having a consult with the OP of which 14 received additional treatment, as retrieved from questionnaires completed by the OP [16]. The focus of this IPS is the early timing - before SA occurs rather than the type of intervention. The control group received care as usual (ie, when there was a need). A detailed overview of this preventive intervention can be found at Kant et al [16]. 


\section{RCT II}

The selection process for RCT II started in 2007, with 9157 employees responding to the study invitation. Employees were eligible if they were classified as being at high risk for future LTSA and additionally had mild depressive complaints. Depressive complaints were assessed using the depression subscale of the Hospital Anxiety and Depression Scale (HAD-D) which consists of 7 items ranging from 0-21 [33]. The employees were classified as having mild depressive complaints when they scored $\geq 8$ points on the HAD-D. Exclusion criteria were: fully or partly absent from work, already receiving treatment by the psychologist/psychiatrist at the time of completing the screening questionnaire, pregnant or on maternity leave. This resulted in $\mathrm{N}=139$ employees who were eligible for randomization in the intervention or control groups. Lexis et al [17] described RCT II in detail.

The original follow-up period for RCT II was 12 months, extended to five years for the current study. The number of study participants decreased over time due to termination of the employment contract as a result of pension, disability benefits, voluntary leave or, involuntary leave. A flow diagram of study participants is shown in online appendix S2.

Employees in the intervention group received a psychological treatment based on principles of PST and CBT to enhance their coping ability to prevent LTSA and stimulate personal well-being. Seven individual sessions of 45 minutes each were provided. After each session, homework assignments were given to the employees and discussed in the following session. The number of sessions could be extended to 13 sessions if needed. Ten psychologists conducted the sessions and received a 2-day training session before the intervention and a 1-day booster session during the study [17]. The focus of this IPS is the early timing - before SA occurs - as well as the intensity of the individual sessions. Employees in the control group received care a usual.

\section{Outcome measures}

Primary outcome

Indicators of labor participation were investigated by SA parameters, which entailed the mean duration of SA (including $>28$ SA days), SA frequency, the percentage of LTSA (>28 days SA), and the time until the first onset of LTSA. The percentage of LTSA was calculated for each year separately even if the period of LTSA has started the previous year. The occupational health service from a financial service provider 'Beter', provided us with SA data through record linkage on an individual level with company sick-leave registries and anonymized according to the current General Data Protection Regulation. SA duration was measured in both RCT in calendar days according to the defined time window: 1-5 years of follow-up. 


\section{Secondary outcome}

Termination of the employment contract was characterized by the time (in months) until an employee departed the company during the follow-up period. Termination of employment could be due to disability, retirement, job loss, or voluntary leave. The HR office from the company under study provided us with the termination of employment contract dates. Especially the relation between IPS for RCT II employees might be of interest, while work disability studies have shown a strong relation with coping abilities and return to work behavior [30,35]. Termination of the employment contract was perceived to be important, especially for RCT II employees, where the preventive intervention was developed to improve their coping abilities. Further it was investigated if SA and LTSA were precursors for the time until termination of the employment contract.

\section{Statistical analysis}

The indicators of labor participation were analyzed according to the intention-to-treat (ITT) principle. Per protocol analyses are provided in the supplementary material. Poisson regression was used to estimate the efficacy of the IPS in terms of the mean duration of SA, SA frequency, and the percentage of LTSA. The time until the first onset of LTSA and the time until termination of the employment contract was examined with multivariate Cox regression analyses. All analyses were conducted for RCT I and RCT II separately. The analyses were adjusted for the following covariates: age, gender, job function/education level (available data differed between RCT I and RCT II), and long-term illness previous to the screening questionnaire. These covariates were chosen due to their important predicting ability for SA $[36,37]$. A Chi-square test was used to investigate if SA or LTSA were precursors for the departure of employees from the company. All analyses were conducted per year for five years of follow-up, except for the multivariate Cox regression which was estimated for five years of follow-up. All analyses were conducted with the use of SPSS version 25 (IBM Corp, Armonk, NY, USA).

\section{Results}

Baseline characteristics of the participants from RCT I and II are displayed in table 1. Age, mean number of years working for the company and working hours per week were similar for the control and intervention groups. Small differences were apparent with regards to gender, educational level, and long-term illness. 


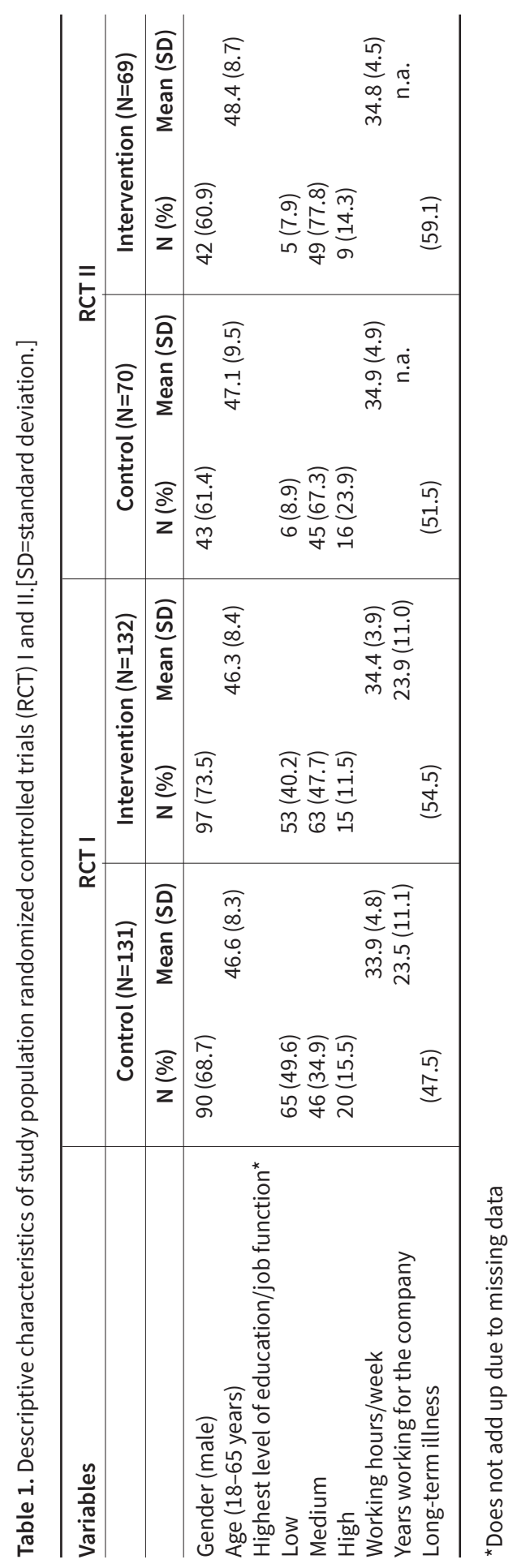


Table 2. Overview efficacy of the indicated prevention strategy on sickness absence parameters for randomized controlled trial I (intention to treat analyses). [LTSA=long-term sickness absence; SD=standard deviation.]

\begin{tabular}{|c|c|c|c|c|c|c|c|c|c|c|c|c|}
\hline \multirow[t]{2}{*}{ Follow-up period } & \multicolumn{4}{|c|}{ Control group } & \multicolumn{4}{|c|}{ Intervention group } & \multicolumn{2}{|c|}{ Difference } & \multirow[t]{2}{*}{ P-value a } & \multirow[t]{2}{*}{ P-value ${ }^{b}$} \\
\hline & Mean (SD) & $\%$ & Median & $\mathrm{N}$ & Mean (SD) & $\%$ & Median & $\mathrm{N}$ & Mean & $\%$ & & \\
\hline \multicolumn{13}{|l|}{ 2-years } \\
\hline Total SA duration $^{c}$ & $68.4(88.1)$ & & 35.8 & 114 & $55.5(76.4)$ & & 24.0 & 125 & 12.9 & & 0.164 & 0.08 \\
\hline SA frequency & $5.42(5.47)$ & & 4.0 & 114 & $4.85(3.83)$ & & 4.0 & 125 & 0.57 & & 0.297 & 0.089 \\
\hline Percentage of LTSA ${ }^{d}$ & & 26.7 & & 35 & & 23.5 & & 31 & & 3.2 & 0.306 & 0.225 \\
\hline \multicolumn{13}{|l|}{ 3-years } \\
\hline Total SA duration ${ }^{c}$ & $104.3(119.8)$ & & 56.6 & 109 & $82.2(105.9)$ & & 36.02 & 119 & 22.1 & & 0.099 & 0.069 \\
\hline SA frequency & $7.66(7.38)$ & & 6.0 & 109 & $6.89(5.30)$ & & 6.0 & 119 & 0.77 & & 0.313 & 0.078 \\
\hline Percentage of LTSA d & & 36.6 & & 48 & & 24.2 & & 32 & & 12.4 & 0.011 & 0.005 \\
\hline \multicolumn{13}{|l|}{ 4-years } \\
\hline Total SA duration ${ }^{c}$ & $125.2(150.3)$ & & 65.0 & 99 & $112.5(150.5)$ & & 49.5 & 114 & 12.7 & & 0.485 & 0.376 \\
\hline SA frequency & $9.36(9.44)$ & & 7.0 & 99 & $8.62(6.36)$ & & 7.0 & 114 & 0.74 & & 0.450 & 0.091 \\
\hline Percentage of LTSA ${ }^{d}$ & & 35.1 & & 46 & & 27.3 & & 36 & & 7.8 & 0.042 & 0.025 \\
\hline \multicolumn{13}{|l|}{ 5-years } \\
\hline Total SA duration $^{c}$ & $166.3(202.7)$ & & 93.5 & 94 & $123.1(167.1)$ & & 65.0 & 101 & 43.2 & & 0.06 & 0.055 \\
\hline SA frequency & $11.44(11.18)$ & & 9.0 & 94 & $10.64(7.59)$ & & 9.0 & 101 & 0.80 & & 0.522 & 0.135 \\
\hline Percentage of LTSA ${ }^{d}$ & & 35.9 & & 47 & & 24.2 & & 32 & & 11.7 & 0.019 & 0.007 \\
\hline
\end{tabular}

${ }^{a}$ Crude analysis using Poisson regression without adjustments for covariates.

${ }^{\mathrm{b}}$ Adjusted analysis using Poisson regression for covariates: age, gender, job function and long-term illness.

c Total SA duration including $>28$ days SA.

d Percentage LTSA is calculated annually. 


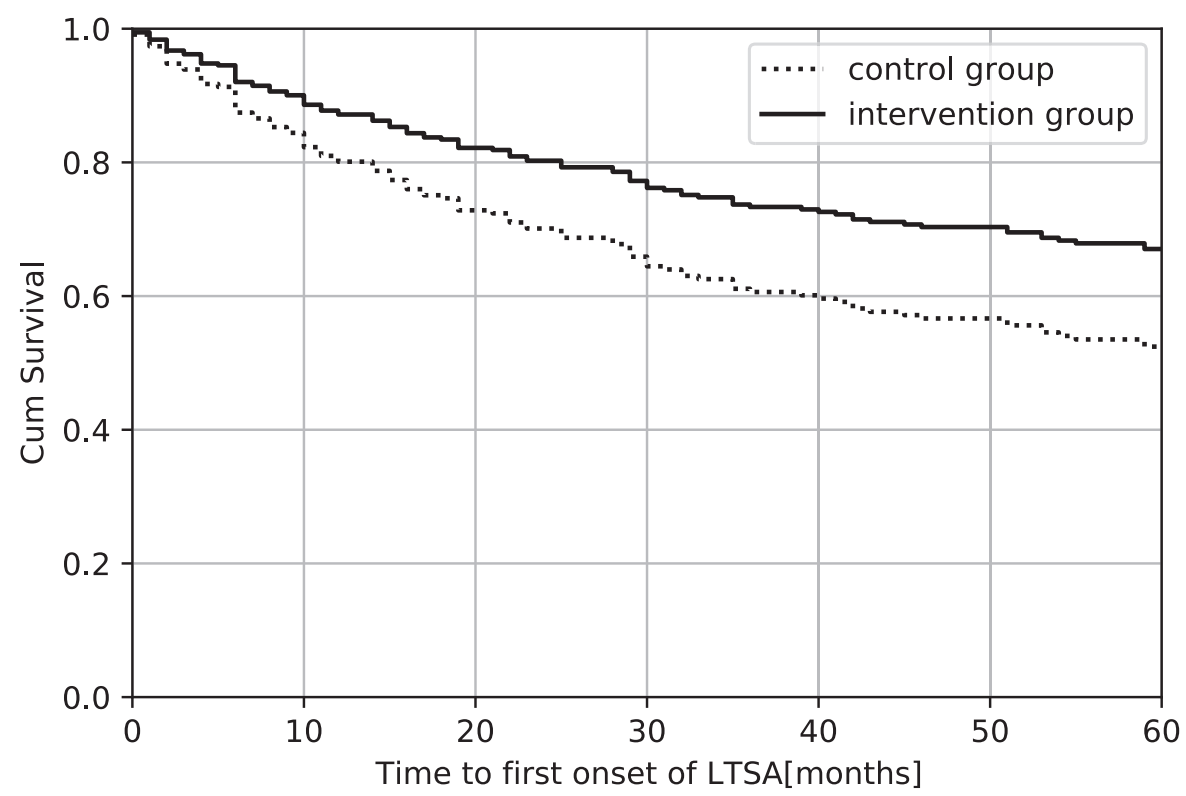

Figure 1A. Time till first spell of LTSA (RCT I) according to the intention to treat principle (HR $0.61 ; 95 \% \mathrm{Cl}$ 0.41-0.90).

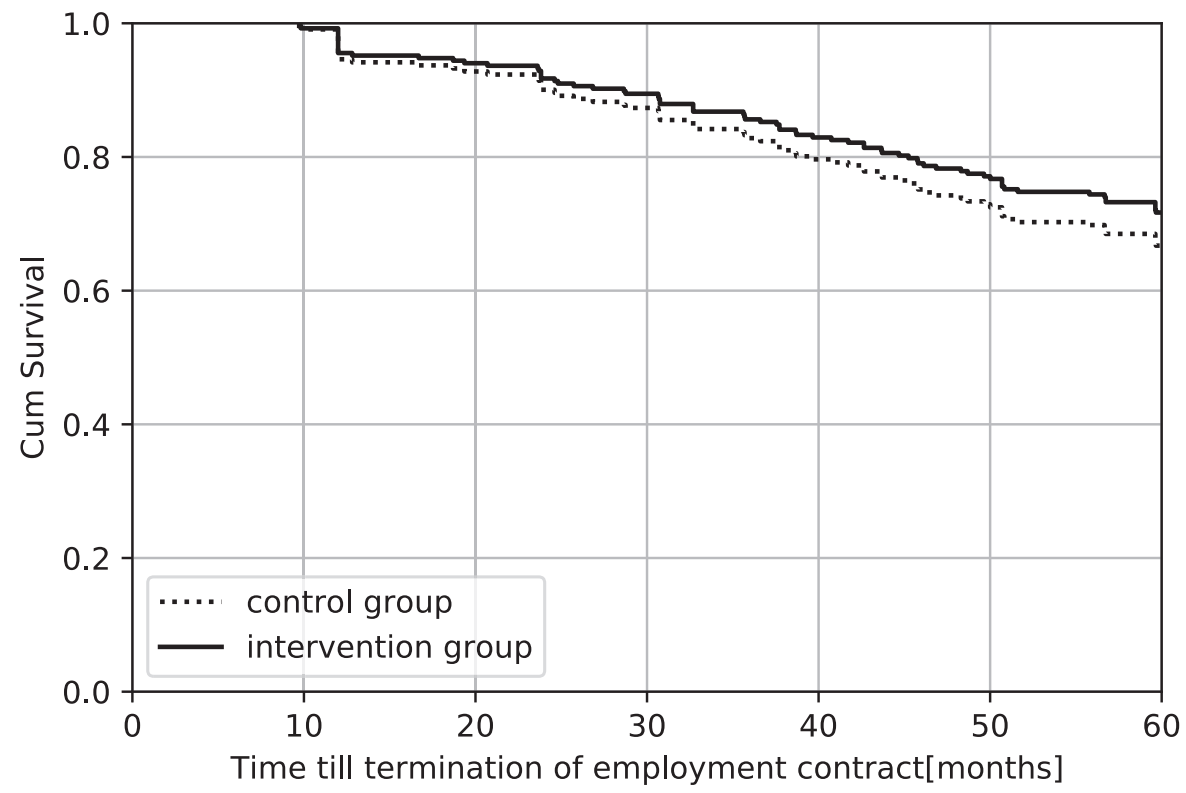

Figure 1B. Time till exit from the company contract in months (RCT I) according to the intention to treat principle (HR 0.85; 95\% Cl 0.54-1.35). 


\section{Results sickness absence parameters and termination of employment contract (RCT I)}

Table 2 presents the results according to the ITT principle for RCT I. On average, the intervention group had fewer mean days of SA in each year compared to the control group. Mean days of SA differed only borderline statistically significant for the five years of follow-up with a difference of 43.2 days of SA between the control and intervention groups. The Per Protocol (PP) analysis showed statistically significant differences between the intervention and control group for each year except four years of follow-up. After five years a difference of 52.8 mean days of SA [95\% confidence interval (CI) 3.21-123.31] between the control and intervention groups in favor of the latter was found. Results from the PP analysis are available in the supplementary material (Table S1, figure S1 and S2). The percentage of LTSA was lower in the intervention compared to control group and was statistically significant after three, four, and five years of follow-up according to both ITT and PP analysis.

The course over time until the first onset of LTSA for the intervention and control groups is shown for RCT I in figure $1 \mathrm{~A}$. According to this survival curve of figure $1 \mathrm{~A}$, after five years, $35 \%$ of the intervention group was on sick leave for $>28$ days as compared to $50 \%$ of the control group. For the intervention group, this resulted in an average time until the first onset of LTSA of 42.3 months compared to 36.1 months for the control group. According to the ITT principle, adjusted for the covariates age, gender, job function, and long-term illness this gave a hazard ratio (HR) of 0.61 (95\% Cl 0.41-0.90).

The average time until termination of the employment contract was 53.8 and 51.8 months for the intervention and control groups, respectively, HR 0.85 (95\% Cl 0.54-1.35) (adjusted, figure 1B). Employees who left the company during the five years' follow-up did not differ statistically significant in terms of days of SA or LTSA from those who did not leave the company.

\section{Results sickness absence parameters and termination of employment contract (RCT II)}

The SA results for RCT II according to the ITT are presented in table 3. No differences were found in SA duration and frequency between the intervention and control group according to the ITT and PP analysis. Cumulative after 3-5 years, the control group had less SA days compared to the intervention group, however this evidence is very uncertain and not statistically significant according to both ITT and PP analysis. Results from the PP analysis are available in the supplementary material (Table S2, figure S3 and S4).

The average time until the first onset of LTSA was 38.1 and 33.1 months for the intervention and control groups, respectively, as shown in figure $2 \mathrm{~A}$. However, this difference was not statistically significant given the adjusted HR 1.31 (95\% Cl 0.70-2.47). 
Table 3. Overview efficacy of the indicated prevention strategy on sickness absence parameters for randomized controlled trial I (intention to treat analyses). [LTSA=long-term sickness absence; SD=standard deviation.]

\begin{tabular}{|c|c|c|c|c|c|c|c|c|c|c|c|c|}
\hline \multirow[t]{2}{*}{ Follow-up period } & \multicolumn{4}{|c|}{ Control group } & \multicolumn{4}{|c|}{ Intervention group } & \multicolumn{2}{|c|}{ Difference } & \multirow[t]{2}{*}{ P-value a } & \multirow[t]{2}{*}{ P-value ${ }^{b}$} \\
\hline & Mean (SD) & $\%$ & Median & $\mathrm{N}$ & Mean (SD) & $\%$ & Median & $\mathrm{N}$ & Mean & $\%$ & & \\
\hline \multicolumn{13}{|l|}{ 2-years } \\
\hline Total SA duration $^{c}$ & $81.2(114.2)$ & & 29.5 & 56 & $71.1(109.0)$ & & 29.0 & 65 & 10.1 & & 0.555 & 0.554 \\
\hline SA frequency & $3.68(2.88$ & & 3.0 & 56 & $3.68(2.83)$ & & 3.0 & 65 & 0 & & 0.997 & 0.748 \\
\hline Percentage of LTSA d & & 18.6 & & 13 & & 21.7 & & 15 & & -3.1 & 0.985 & 0.975 \\
\hline \multicolumn{13}{|l|}{ 3-years } \\
\hline Total SA duration $^{\mathrm{c}}$ & $106.6(135.8)$ & & 43.5 & 50 & $110.5(143.0)$ & & 57.0 & 60 & -3.9 & & 0.868 & 0.996 \\
\hline SA frequency & $5.46(4.04)$ & & 4.5 & 50 & $5.47(4.39)$ & & 4.0 & 60 & -0.01 & & 0.993 & 0.587 \\
\hline Percentage of LTSA d & & 21.4 & & 15 & & 30.4 & & 21 & & -9 & 0.597 & 0.695 \\
\hline \multicolumn{13}{|l|}{ 4-years } \\
\hline Total SA duration ${ }^{\mathrm{c}}$ & $133.0(185.2)$ & & 36.5 & 40 & $145.7(185.1)$ & & 74.8 & 58 & -12.7 & & 0.731 & 0.891 \\
\hline SA frequency & $6.45(5.42)$ & & 5.0 & 40 & $6.76(5.07)$ & & 5.5 & 58 & -0.31 & & 0.758 & 0.930 \\
\hline Percentage of LTSA d & & 17.1 & & 12 & & 30.4 & & 21 & & -13.3 & 0.547 & 0.746 \\
\hline \multicolumn{13}{|l|}{ 5-years } \\
\hline Total SA duration ${ }^{c}$ & $170.5(249.0)$ & & 54.1 & 33 & $197.6(251.3)$ & & 98.0 & 47 & -27.05 & & 0.596 & 0.776 \\
\hline SA frequency & $7.15(5.97)$ & & 6.0 & 33 & $8.08(5.83)$ & & 7.0 & 47 & -0.93 & & 0.459 & 0.777 \\
\hline Percentage of LTSA ${ }^{d}$ & & 17.1 & & 12 & & 29.0 & & 20 & & -11.9 & 0.619 & 0.786 \\
\hline
\end{tabular}

a Crude analysis using Poisson regression without adjustments for covariates.

${ }^{\mathrm{b}}$ Adjusted analysis using Poisson regression for covariates; age, gender, education level and long-term illness.

c Total SA duration including $>28$ days SA.

d Percentage LTSA is calculated annually. 


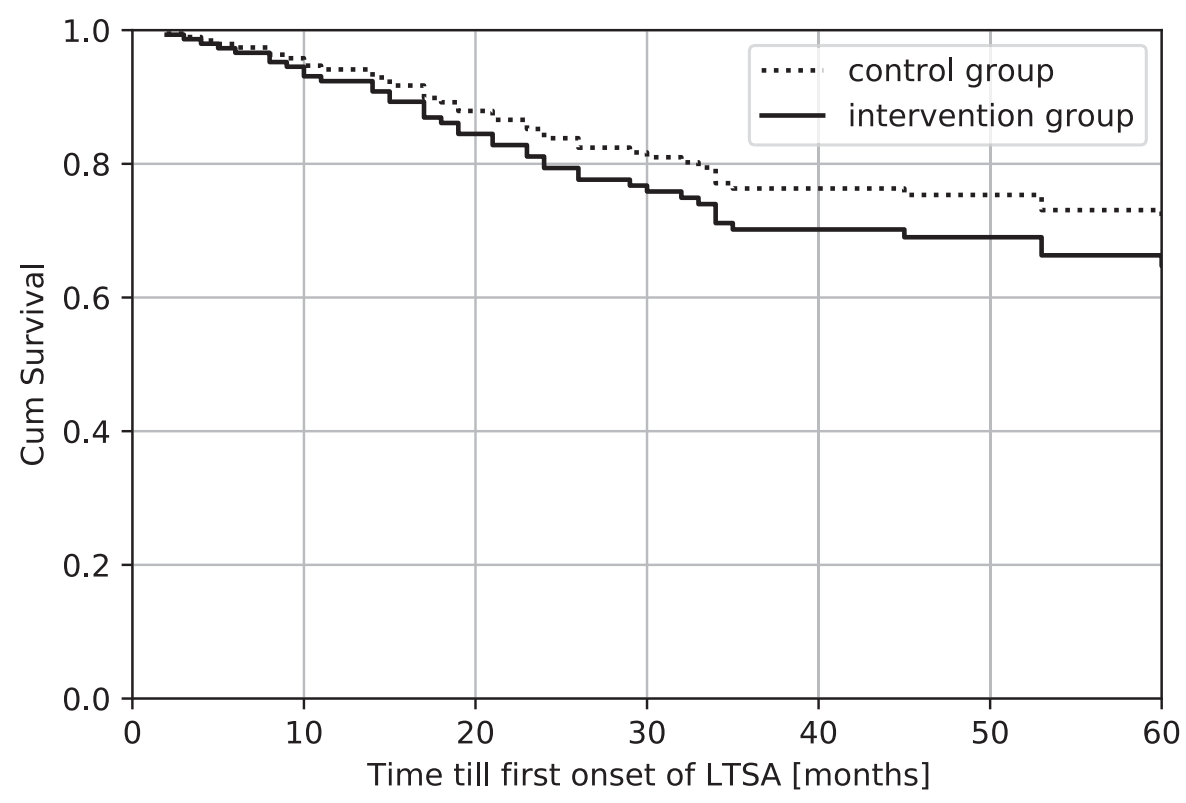

Figure 2A. Time till first onset of LTSA (RCT II intention to treat principle) (HR 1.31, 95\% Cl 0.70-2.47).

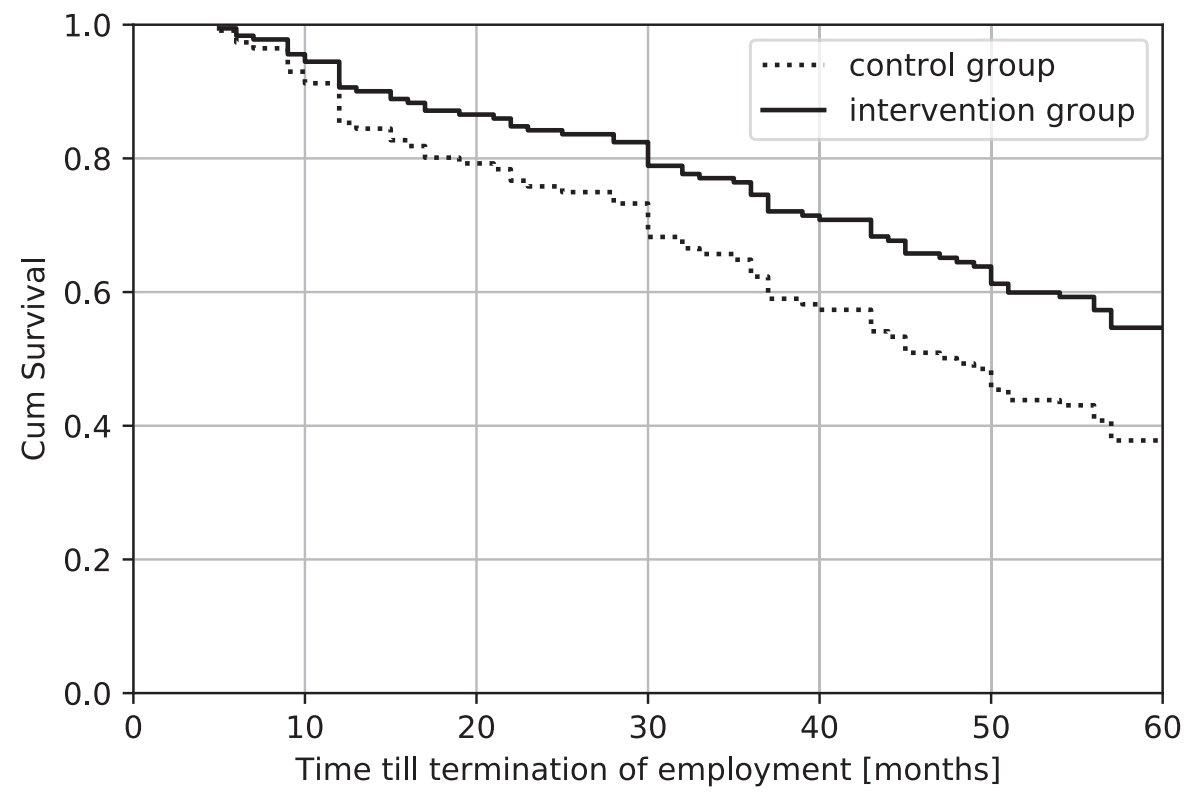

Figure 2B. Time till termination of the employment contract with the company in months (RCT II intention to treat) (HR 0.62, 95\% $\mathrm{Cl} 0.39-0.99$ ). 
The average time until termination of the employment contract for the intervention group was 48.7 months and for the control group 40.2 months. As shown in Figure 2B, according to the ITT principle, after five years $45 \%$ of the intervention group as compared to $62 \%$ of the control group departed the company. The difference between the groups was significant with a HR $0.62(95 \% \mathrm{Cl}$ 0.39-0.99) (adjusted). According to the PP analysis, 35\% of the intervention group departed from the company as compared to $60 \%$ from the control group with a HR $0.48(95 \% \mathrm{Cl} 0.27-0.85)$ (adjusted, see S4). The employees who departed the company did not differ statistically significant from the employees who stayed with the company during the five years in terms of SA and LTSA.

\section{Discussion}

The study aimed to estimate the efficacy of an IPS on the prevention of LTSA and termination of the employment contract over five years of follow-up. This was a followup study on employees from two Dutch RCT with an original follow-up of 12 months. The focus was on employees classified by a screening questionnaire as being at high risk for future LTSA. Additionally, RCT II only included employees with mild depressive complaints. The RCT also differed in type and treatment intensity. Previous results from 12 months' follow-up showed statistically significant reductions in days of SA in favor of the intervention group (RCT I and RCT II), as well as a reduction of depressive symptoms in the intervention group (RCT II).

This study showed over five years that, on average, the intervention group (RCT I) had 43.1 fewer days of SA compared to the control group $(P=0.05)$ and showed significantly less LTSA (24.2\% versus $35.9 \% ; P=0.019)$. The intervention group had a significantly longer time until their first period of LTSA (42.3 versus 36.1 months) (HR 0.61, 95\% Cl 0.41-0.90). Termination of the employment contract did not differ between the control and intervention group. For RCT II participants, no significant differences in days of SA and LTSA were found between the groups after 12 months of follow-up. The time until the first onset of LTSA was somewhat longer in the intervention compared to control group over five years (HR 1.31, 95\% Cl 0.702.47). Whereas, the risk of termination of the employment contract was significantly lower in the intervention group ( $\mathrm{HR} 0.62,95 \% \mathrm{Cl} 0.39-0.99$ ).

Only six other studies to date reported results of an IPS on SA or work-related outcomes [20-22, 38-40]. However, none of these reported outcomes beyond 24 months of followup, and five of the six only covered at most one year follow-up. This means that beyond the two RCT described here, comparison with other results from studies describing longterm effects (five years) is not possible. The comparison of our study results with other preventive approaches aimed at SA, not based on indicated prevention, is also challenging 
as the majority of these studies either focused on the general population or employees already on sick leave and showed different results [41-43]. The 5-year follow-up of our study with regards to the efficacy of LTSA or work disability interventions seems rather unique, as even with a general focus of studies on SA reduction, no other studies were found for comparison.

The 5-year study period was chosen due to the expected long-term effects as potential changes in help-seeking behavior might occur among the employees at high-risk for future SA and subsequently could lower the threshold to visit a physician, especially visits to an OP, who can also advise in adjustments to the work situation or stimulate addressing potential issues with a supervisor, management or colleagues [23]. The healthcare usage is currently being analyzed and the preliminary results show that the IPS for both RCT increases short-term healthcare usage (Klasen et al, unpublished). Regrettably, due to privacy reasons, no further information could be retrieved on the content and number of consults with the OP and the decisions made. Therefore, one can only hypothesize that the early consult with the OP led to a long-lasting decrease in SA due to the early awareness of a health problem/personal issue. But also the process of problem identification and the drafting of a concrete plan of action by the OP might have contributed in long lasting effects as reported. However, further studies should ideally verify these results in similar and other study populations.

The results of RCT II were unexpected, as no decrease in SA was observed during 2-5 years follow-up. Due to the long-term results from CBT regarding depressive complaints, as reported in several long-term studies (covering 3-6 years), one might expect that a more lasting effect would exist also for SA [28-30]. However, in the current study, no evidence was found for a sustainable decrease in SA after one year. It might be that the number of subjects was insufficient to find potential small effects. Additionally, the difference in efficacy of long-term effects of CBT interventions might be the result of more severe depressive complaints as compared to the less severe ones of the current study participants [29]. Furthermore, the current study did not include booster sessions during the prolonged follow-up, which is expected to have led to better outcomes [28]. Further studies might focus more on the sustainable spectrum of effects on the early consultation with the OP and the PST/CBT intervention and investigate the efficacy elements of each strategy.

The efficacy differences between the RCT might at least partially be due to the different selection criteria of RCT participants. In addition to being at high risk for future SA, RCT II participants were selected for experiencing mild depressive complaints. Therefore, other factors might be of importance for an approach to prevent future SA compared to the general high-risk population. Depressive complaints might give rise to, eg, stigmatization, lower socioeconomic status, loss of a valuable source of social support [44, 45]. Possibly, more holistic care is needed for these people, while their healthcare needs will be larger, 
due to different health and personal influences as a result of their illness. A difference in efficacy may also be explained by different intervention characteristics. RCT I focused on issues that emerged from the early consultation with a suited intervention, while RCT II was based on a psychological treatment with principles of PST/CBT and was developed to improve employees coping skills. Moreover, the intervention in RCT II involved many different sessions and was, therefore, more intense than RCT I.

Thisisthefirststudy, asfarasweknow which investigated thetermination of theemployment contract in an indicated preventive setting. In general preventive interventions, it was often studied as work disability or workability $[46,47]$. The termination of employment contract in this study is less specific, it could be due to disability, retirement, job loss, or voluntary leave and is therefore difficult to compare to other studies. Although in RCT II the risk of termination of employment was found to be substantially lower in favor of the intervention group, we do not have a clear explanation for this positive and relevant effect. Possibly, PST/CBT makes the intervention group more resilient and more proactive with regards to solving potential participation problems.

The strengths of this study are its randomized and longitudinal design, objective measurement of SA, and termination of the employment contract, data availability of two $\mathrm{RCT}$, and no large differences in the ITT and PP outcomes within the RCT were observed. The termination of the employment contract dates provided by the company is expected to be the golden standard [48]. There was no differential loss to follow-up, which resulted in an even comparison over the years. Employees who left the company did not differ in terms of SA and LTSA compared to those who stayed and, therefore, we expect it did not have a large impact on the average days of SA per year. As one might assume that censored employees have similar prospects of reaching the outcome as those who continued to be followed, bias of the survival analysis due to right-censoring is assumed to be low. The researcher who analyzed the data was blinded due to anonymized personnel numbers. Contamination in the first three years of study was not observed while the control group did not receive an early OP consultation. Possibly other healthcare or interventions were used but, in the strict sense of the early consultation as being an essential part of the intervention, these were not seen as co-interventions.

It is conceivable that contamination between the intervention and control groups occurred as both groups received the IPS according to the protocol of the first RCT after three years (if identified as high risk yet again). Unfortunately, we were not able to retrieve if, and how often, this occurred. This might have reduced the contrast between the groups and most likely this bias has led to an underestimation of the efficacy, assuming the intervention is effective.

The underlying reason for the termination of the employment contract was unavailable to the researchers due to the EU's strict General Data Protection Regulation. No distinction 
could be made between the termination of the employment contract due to disability, retirement, job loss or, voluntary leave. Therefore, the results from this study may be seen as the first step towards better understanding the efficacy of an IPS on termination of the employment contract, and future studies may further distinguish the reasons for leaving the company.

In addition, a technical malfunction in the data merge resulted in a loss to follow-up in the first year, of $\mathrm{N}=14$ for RCT I and $\mathrm{N}=10$ for RCT II. There were no indications that this loss to follow-up was selective and unlikely to be related to the outcome or the interventions and, therefore, would not have biased the results. The trials were carried out in a large company in The Netherlands in the context of its specific social system and RCT participants were office workers with access to an occupational health service with a very high service level. Although such an indicated preventive approach could be effective in many countries and contexts, extrapolation of the reported results should take the national and labor context into account while SA and its prevention is highly dependent on cultural as well as legislative factors [3]. Thus, adaptation should be done with care, tailoring and testing of the screening and interventions under study. In companies with a lower occupational health care service level, the contrast between intervention and control groups may be different, resulting in higher or lower effect sizes.

For future studies, the first step should be to validate the results from this study in a different study population. Possibly, with additional data gathering concerning healthcare usage or workplace involvement to better understand the effective elements of both interventions. Moreover, future studies should extend their follow-up period to investigate the full potential of their intervention while currently, similar studies often focused on a short period. Furthermore, it would be interesting to investigate if IPS differs in efficacy for mental or physical health complaints and leads to medicalization. The IPS is focused on individual health/general problems and the company was only involved when the employees wanted to express their issues. However, the problems might be related to the organization itself, and possibly involving the organization could improve the shared responsibility for employees' health. Especially the multifactorial factors of LTSA give a lead to a more holistic approach. However, this could encounter inherent difficulties due to privacy issues. Future studies should investigate if a holistic IPS is feasible in an organization where the focus remains on individual treatment, valuing the doctor/patient relationship and privacy issues.

The screening interval of three years of this IPS seems suited to create long-term effects while especially the efficacy of LTSA is visible after two years. However, the screening interval period has not yet been validated, and it would be interesting for future studies to investigate what is acceptable in terms of costs and benefits for both employees and employers. 
To conclude, after one year, the IPS resulted in a large reduction in days of SA. With an extended follow-up of five years, this strategy showed a reduction in days of SA and LTSA for the intervention compared to control group (in RCT I). However, this decrease was not found for participants with mild depressive complaints after the first year (RCT II). For participants receiving a psychological treatment based on PST/CBT, RCT II showed that the intervention had a positive effect on preventing termination of the employment contract. This relation was not found in RCT I. A different type of intervention and study population might have resulted in different results for the RCT. Thus, the best elements of both interventions should be further studied.

\section{Acknowledgements}

RCT I is registered under NTR214. The study protocol of RCT II was submitted to and financially supported by The Netherlands Organisation for Health Research and Development (grant no. 62200024). The RCTs were performed in agreement with the ethical and medical guidelines at the time of the studies. RCT I complied with the Declaration of Helsinki [34]. For RCT II, study approval was provided by the Medical and Ethical Committee of Maastricht University (MEC 06-3-018), which was not applicable at the time of the RCT I. 


\section{References}

1. Labriola M. Conceptual framework of sickness absence and return to work, focusing on both the individual and the contextual level. Work 2008;30(4):377-87.

2. Lund T, Kivimäki M, Labriola M, Villadsen E, Christensen KB. Using administrative sickness absence data as a marker of future disability pension: the prospective DREAM study of Danish private sector employees. Occup Environ Med 2008 Jan;65(1):28-31. https://doi. org/10.1136/oem.2006.031393.

3. van Amelsvoort LG, Jansen NW, Kant I. Addressing long-term sickness absence: moving beyond disease, illness and work-related factors for effective prevention. Scand J Work Environ Health 2017 Jan;43(1):1-4. https://doi.org/10.5271/sjweh.3605.

4. Helgesson M, Johansson B, Nordqvist T, Lundberg I, Vingård E. Sickness absence at a young age and later sickness absence, disability pension, death, unemployment and income in native Swedes and immigrants. Eur J Public Health 2015 Aug;25(4):688-92. https://doi.org/10.1093/ eurpub/cku250.

5. Vingård E, Alexanderson K, Norlund A. Chapter 9. Consequences of being on sick leave. Scand J Public Health. 2004;32(63_suppl):207-15.

6. Wiencke M, Cacace M, Fischer S. Healthy at Work: Interdisciplinary Perspectives: Springer; 2016.

7. van Rijn RM, Robroek SJ, Brouwer S, Burdorf A. Influence of poor health on exit from paid employment: a systematic review. Occup Environ Med 2014 Apr;71(4):295-301. https://doi. org/10.1136/oemed-2013-101591.

8. Waddell G, Burton AK. Concepts of rehabilitation for the management of low back pain. Best Pract Res Clin Rheumatol 2005 Aug;19(4):655-70. https://doi.org/10.1016/j.berh.2005.03.008.

9. Munir F, Yarker J, Haslam C. Sickness absence management: encouraging attendance or 'risktaking' presenteeism in employees with chronic illness? Disabil Rehabil 2008;30(19):146172. https://doi.org/10.1080/09638280701637380.

10. Beemsterboer W, Stewart R, Groothoff J, Nijhuis F. A literature review on sick leave determinants (1984-2004). Int J Occup Med Environ Health 2009;22(2):169-79. https://doi. org/10.2478/v10001-009-0013-8.

11. Notenbomer A, Roelen C, Groothoff J, van Rhenen W, Bültmann U. Effect of an eHealth Intervention to Reduce Sickness Absence Frequency Among Employees With Frequent Sickness Absence: Randomized Controlled Trial. J Med Internet Res 2018 Oct;20(10):e10821. https://doi.org/10.2196/10821.

12. Schultz IZ, Gatchel RJ. Handbook of return to work: From research to practice: Springer; 2015.

13. Vingård E, Lindberg P, Josephson M, Voss M, Heijbel B, Alfredsson L et al. Long-term sick-listing among women in the public sector and its associations with age, social situation, lifestyle, and work factors: a three-year follow-up study. Scand J Public Health 2005;33(5):370-5. https://doi.org/10.1080/14034940510005860. 
14. Andersen LL, Thorsen SV, Flyvholm MA, Holtermann A. Long-term sickness absence from combined factors related to physical work demands: prospective cohort study. Eur J Public Health 2018 Oct;28(5):824-9. https://doi.org/10.1093/eurpub/cky073.

15. Aronsson V, Toivanen S, Leineweber C, Nyberg A. Can a poor psychosocial work environment and insufficient organizational resources explain the higher risk of ill-health and sickness absence in human service occupations? Evidence from a Swedish national cohort. Scand J Public Health 2019 May;47(3):310-7. https://doi.org/10.1177/1403494818812638.

16. Kant I, Jansen NW, van Amelsvoort LG, van Leusden R, Berkouwer A. Structured early consultation with the occupational physician reduces sickness absence among office workers at high risk for long-term sickness absence: a randomized controlled trial. J Occup Rehabil 2008 Mar;18(1):79-86. https://doi.org/10.1007/s10926-007-9114-z.

17. Lexis MA, Jansen NW, Huibers MJ, van Amelsvoort LG, Berkouwer A, Tjin A Ton G et al. Prevention of long-term sickness absence and major depression in high-risk employees: a randomised controlled trial. Occup Environ Med 2011 Jun;68(6):400-7. https://doi. org/10.1136/oem.2010.057877.

18. Duijts SF, Kant IJ, Landeweerd JA, Swaen GM. Prediction of sickness absence: development of a screening instrument. Occup Environ Med 2006 Aug;63(8):564-9. https://doi.org/10.1136/ oem.2005.024521.

19. Salomonsson S, Hedman-Lagerlöf E, Öst LG. Sickness absence: a systematic review and meta-analysis of psychological treatments for individuals on sick leave due to common mental disorders. Psychol Med 2018 Sep;48(12):1954-65. https://doi.org/10.1017/ S0033291718000065.

20. Duijts SF, Kant I, van den Brandt PA, Swaen GM. Effectiveness of a preventive coaching intervention for employees at risk for sickness absence due to psychosocial health complaints: results of a randomized controlled trial. J Occup Environ Med 2008 Jul;50(7):765-76. https:// doi.org/10.1097/JOM.0b013e3181651584.

21. Lerner D, Adler D, Hermann RC, Chang H, Ludman EJ, Greenhill A et al. Impact of a workfocused intervention on the productivity and symptoms of employees with depression. $J$ Occup Environ Med 2012 Feb;54(2):128-35. https://doi.org/10.1097/JOM.0b013e31824409d8.

22. Taimela S, Justén S, Aronen P, Sintonen H, Läärä E, Malmivaara A et al. An occupational health intervention programme for workers at high risk for sickness absence. Cost effectiveness analysis based on a randomised controlled trial. Occup Environ Med 2008 Apr;65(4):242-8. https://doi.org/10.1136/oem.2007.033167.

23. Lexis MA, Jansen NW, Stevens FC, van Amelsvoort LG, Kant I. Experience of health complaints and help seeking behavior in employees screened for depressive complaints and risk of future sickness absence. J Occup Rehabil 2010 Dec;20(4):537-46. https://doi.org/10.1007/ s10926-010-9244-6.

24. Steenbeek R. Workers' opinions on the effect of contact with health care providers on sickness 
absence duration. Work 2014;49(3):495-504. https://doi.org/10.3233/WOR-131714.

25. Mortelmans AK, Donceel P, Lahaye D, Bulterys S. Work-related sickness absences and mandatory occupational health surveillance. Occup Med (Lond) 2008 Oct;58(7):464-7. https://doi.org/10.1093/occmed/kqn089.

26. Driessen E, Hollon SD. Cognitive behavioral therapy for mood disorders: efficacy, moderators and mediators. Psychiatr Clin North Am 2010 Sep;33(3):537-55. https://doi.org/10.1016/j. psc.2010.04.005.

27. Hollon SD. Does cognitive therapy have an enduring effect? Cognit Ther Res 2003;27(1):71-5. https://doi.org/10.1023/A:1022538713914.

28. Fava GA, Ruini C, Rafanelli C, Finos L, Conti S, Grandi S. Six-year outcome of cognitive behavior therapy for prevention of recurrent depression. Am J Psychiatry 2004 Oct;161(10):1872-6. https://doi.org/10.1176/ajp.161.10.1872.

29. Bockting CL, Spinhoven P, Wouters LF, Koeter MW, Schene AH; DELTA Study Group. Longterm effects of preventive cognitive therapy in recurrent depression: a 5.5-year follow-up study. J Clin Psychiatry 2009 Dec;70(12):1621-8. https://doi.org/10.4088/JCP.08m04784blu.

30. Øyeflaten I, Midtgarden IJ, Maeland S, Eriksen HR, Magnussen LH. Functioning, coping and work status three years after participating in an interdisciplinary, occupational rehabilitation program. Scand J Public Health 2014 Jul;42(5):425-33. https://doi. org/10.1177/1403494814528291.

31. Nigatu YT, Huang J, Rao S, Gillis K, Merali Z, Wang J. Indicated Prevention Interventions in the Workplace for Depressive Symptoms: A Systematic Review and Meta-analysis. Am J Prev Med 2019 Jan;56(1):e23-33. https://doi.org/10.1016/j.amepre.2018.08.027.

32. Kant I, Jansen NW, van Amelsvoort LG, Swaen GM, van Leusden R, Berkouwer A. Screening questionnaire Balansmeter proved successful in predicting future long-term sickness absence in office workers. J Clin Epidemiol 2009;62(4):408-14. e2.

33. Zigmond AS, Snaith RP. The hospital anxiety and depression scale. Acta Psychiatr Scand 1983 Jun;67(6):361-70. https://doi.org/10.1111/j.1600-0447.1983.tb09716.x.

34. Association WM; World Medical Association. World Medical Association Declaration of Helsinki: ethical principles for medical research involving human subjects. JAMA 2013 Nov;310(20):2191-4. https://doi.org/10.1001/jama.2013.281053.

35. Odéen M, Kristensen LO, Ursin H. Coping and return to work: measurement and theoretical issues. Nor Epidemiol 2009;19(2). https://doi.org/10.5324/nje.v19i2.588.

36. Notenbomer A, van Rhenen W, Groothoff JW, Roelen CA. Predicting long-term sickness absence among employees with frequent sickness absence. Int Arch Occup Environ Health 2019 May;92(4):501-11. https://doi.org/10.1007/s00420-018-1384-6.

37. Andrea H, Beurskens AJ, Metsemakers JF, van Amelsvoort LG, van den Brandt PA, van Schayck CP. Health problems and psychosocial work environment as predictors of long term sickness absence in employees who visited the occupational physician and/or general practitioner in 
relation to work: a prospective study. Occup Environ Med 2003 Apr;60(4):295-300. https://doi. org/10.1136/oem.60.4.295.

38. Adler DA, Lerner D, Visco ZL, Greenhill A, Chang H, Cymerman E et al. Improving work outcomes of dysthymia (persistent depressive disorder) in an employed population. Gen Hosp Psychiatry 2015 Jul-Aug;37(4):352-9. https://doi.org/10.1016/j.genhosppsych.2015.04.001.

39. de Boer AG, van Beek JC, Durinck J, Verbeek JH, van Dijk FJ. An occupational health intervention programme for workers at risk for early retirement; a randomised controlled trial. Occup Environ Med 2004 Nov;61(11):924-9. https://doi.org/10.1136/oem.2003.009746.

40. Wang PS, Simon GE, Avorn J, Azocar F, Ludman EJ, McCulloch J et al. Telephone screening, outreach, and care management for depressed workers and impact on clinical and work productivity outcomes: a randomized controlled trial. JAMA 2007 Sep;298(12):1401-11. https://doi.org/10.1001/jama.298.12.1401.

41. Aasdahl L, Pape K, Vasseljen O, Johnsen R, Gismervik S, Halsteinli V et al. Effect of Inpatient Multicomponent Occupational Rehabilitation Versus Less Comprehensive Outpatient Rehabilitation on Sickness Absence in Persons with Musculoskeletal- or Mental Health Disorders: A Randomized Clinical Trial. J Occup Rehabil 2018 Mar;28(1):170-9. https://doi. org/10.1007/s10926-017-9708-z.

42. Milligan-Saville JS, Tan L, Gayed A, Barnes C, Madan I, Dobson M et al. Workplace mental health training for managers and its effect on sick leave in employees: a cluster randomised controlled trial. Lancet Psychiatry 2017 Nov;4(11):850-8. https://doi.org/10.1016/S22150366(17)30372-3.

43. Beiwinkel T, Eißing T, Telle NT, Siegmund-Schultze E, Rössler W. Effectiveness of a Web-Based Intervention in Reducing Depression and Sickness Absence: Randomized Controlled Trial. J Med Internet Res 2017 Jun;19(6):e213. https://doi.org/10.2196/jmir.6546.

44. Lépine JP, Briley M. The increasing burden of depression. Neuropsychiatr Dis Treat 2011;7 Suppl 1:3-7.

45. Plaisier I, Beekman AT, de Bruijn JG, de Graaf R, Ten Have M, Smit JH et al. The effect of social roles on mental health: a matter of quantity or quality? J Affect Disord 2008 Dec;111(23):261-70. https://doi.org/10.1016/j.jad.2008.03.007.

46. Oakman J, Neupane S, Proper KI, Kinsman N, Nygård CH. Workplace interventions to improve work ability: A systematic review and meta-analysis of their effectiveness. Scand $\mathrm{J}$ Work Environ Health 2018 Mar;44(2):134-46. https://doi.org/10.5271/sjweh.3685.

47. Midtsundstad TI, Nielsen RA. Do workplace interventions reduce disability rates? Occup Med (Lond) 2016 Dec;66(9):691-7. https://doi.org/10.1093/occmed/kqw169.

48. Stapelfeldt CM, Jensen C, Andersen NT, Fleten N, Nielsen CV. Validation of sick leave measures: self-reported sick leave and sickness benefit data from a Danish national register compared to multiple workplace-registered sick leave spells in a Danish municipality. BMC Public Health 2012 Aug;12(1):661. https://doi.org/10.1186/1471-2458-12-661. 


\section{CHAPTER 4 - APPENDIX}

\section{Screening questionnaire}

The questionnaire entailed 34 multiple choice questions regarding demographics, work environment, private situation, (mental) health, and SA history. The screening instrument was used to predict which employees were at risk for SA, this resulted for RCT I in a specificity of the Balansmeter of $94.4 \%$ and a sensitivity of $49.4 \%$. For women, a cutoff point with $94.7 \%$ specificity and $30.3 \%$ sensitivity was applied (16). For RCT II, the specificity for men was $87.8 \%$ and for women $87.9 \%$ with a sensitivity for men of $65.1 \%$ and for women $52.6 \%(17)$. 


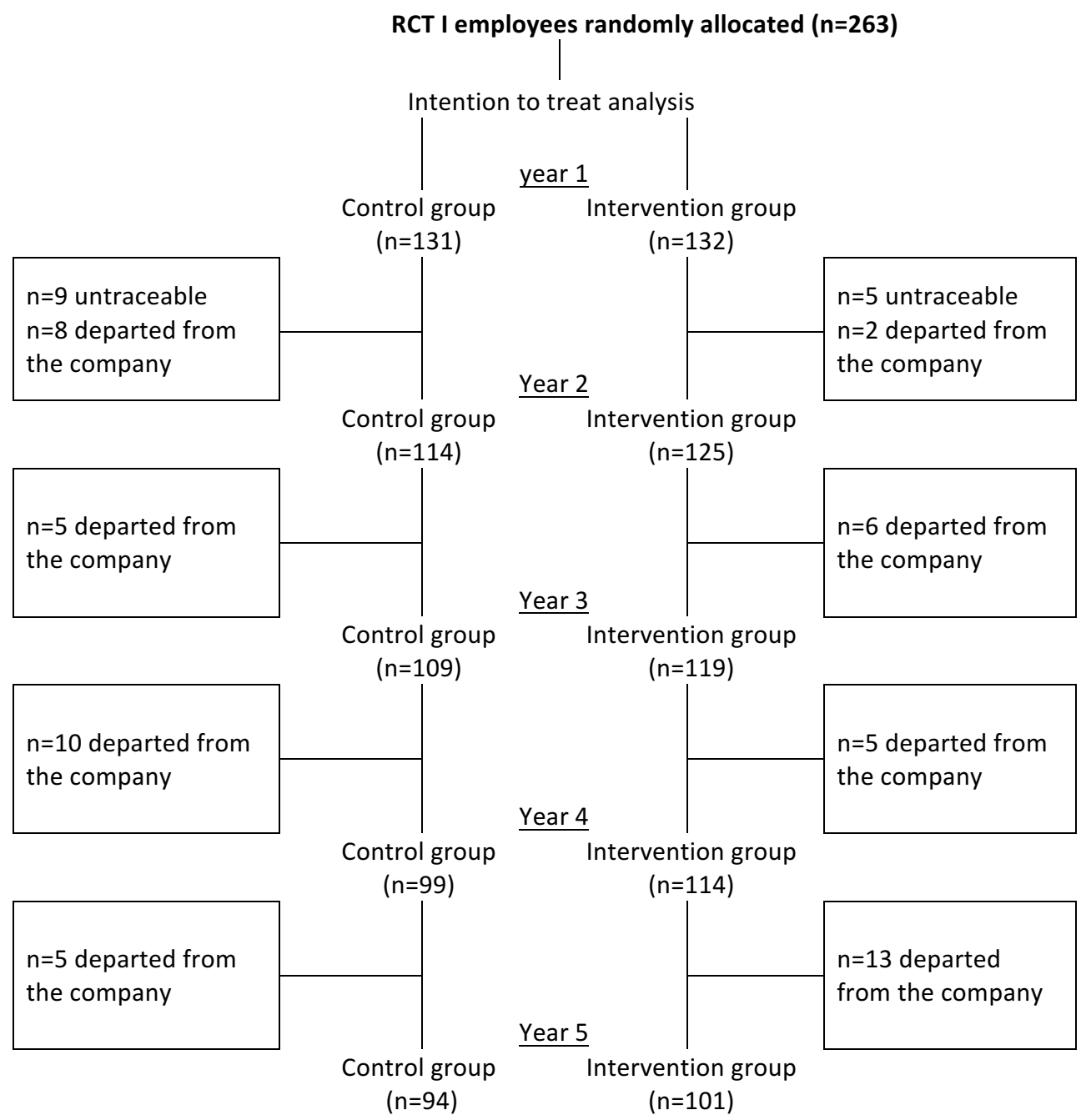

Flow diagram of RCT I participant allocation and departure from the company 


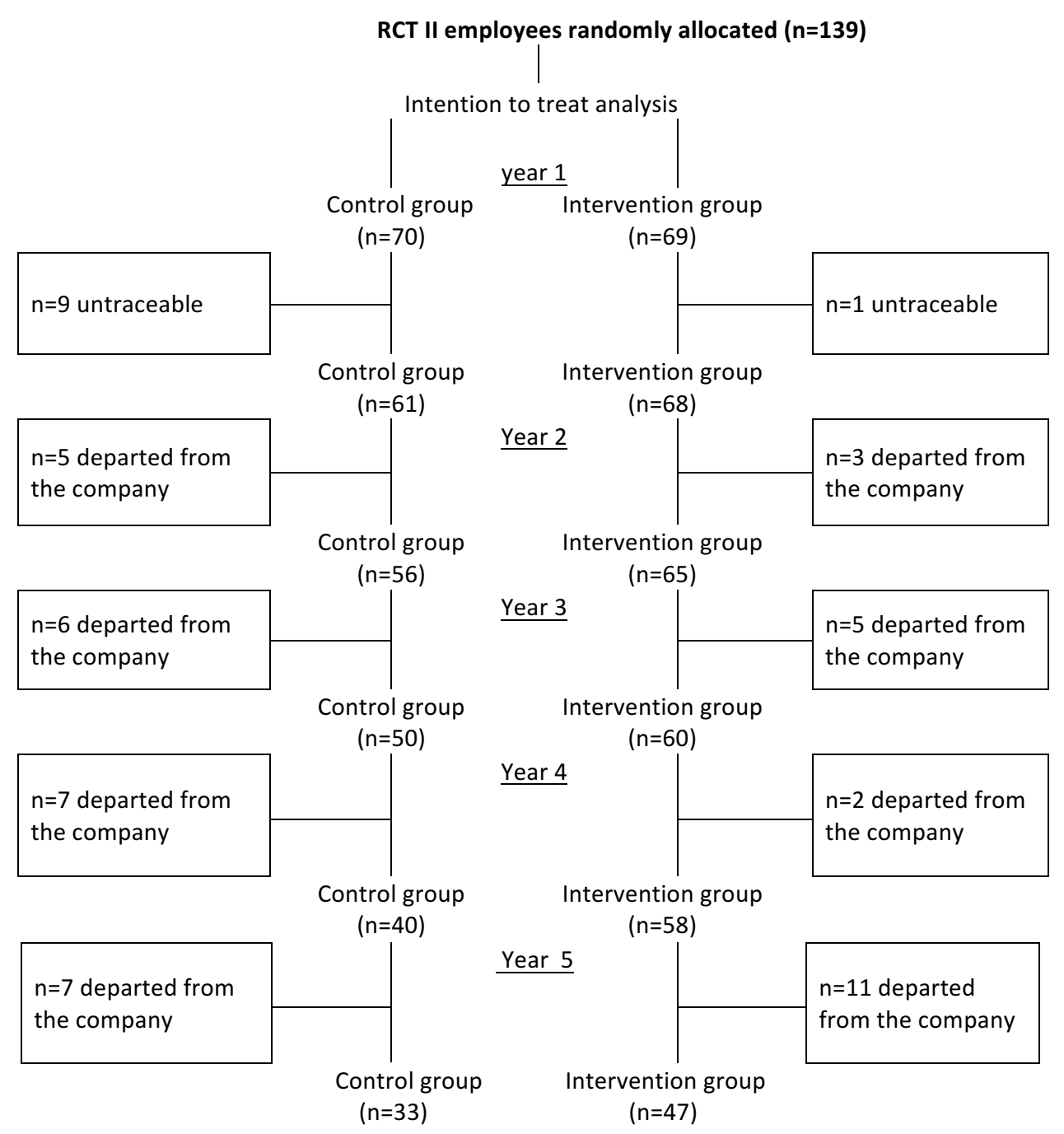

Flow diagram of RCT II participant allocation and departure from the company 
Table S1: Overview efficacy of the indicated prevention strategy on SA parameters for RCT I (per protocol analysis)

\begin{tabular}{|c|c|c|c|c|c|c|c|c|c|}
\hline & \multicolumn{3}{|c|}{ Control group } & \multicolumn{3}{|c|}{ Intervention group } & \multirow{2}{*}{$\begin{array}{c}\text { Mean } \\
\text { Difference }\end{array}$} & \multirow[t]{2}{*}{ P-value ${ }^{1}$} & \multirow[t]{2}{*}{ P-value ${ }^{2}$} \\
\hline & Mean (SD) & Median & $\mathrm{N}$ & Mean (SD) & Median & $\mathrm{N}$ & & & \\
\hline \multicolumn{10}{|l|}{ 2-years follow-up } \\
\hline Total SA duration \pm & $68.4(88.0)$ & 35.8 & 114 & $43.9(62.0)$ & 20.0 & 95 & 24.5 & 0.008 & 0.002 \\
\hline SA frequency & $5.42(5.47)$ & 4.0 & 114 & $4.50(3.45)$ & 4.0 & 95 & 0.92 & 0.114 & 0.020 \\
\hline Percentage of LTSA* & $26.7 \%$ & & 35 & $21.2 \%$ & & 21 & $5.5 \%$ & 0.157 & 0.115 \\
\hline \multicolumn{10}{|l|}{ 3-years follow-up } \\
\hline Total SA duration \pm & $104.3(119.8)$ & 56.6 & 109 & $75.4(105.2)$ & 32.0 & 92 & 28.8 & 0.043 & 0.026 \\
\hline SA frequency & $7.66(7.38)$ & 6.0 & 109 & $6.38(4.89)$ & 5.0 & 92 & 1.28 & 0.111 & 0.015 \\
\hline Percentage of LTSA & $36.6 \%$ & & 48 & $23.2 \%$ & & 23 & $13.4 \%$ & 0.008 & 0.004 \\
\hline \multicolumn{10}{|l|}{ 4-years follow-up } \\
\hline Total SA duration \pm & $125.2(150.4)$ & 65.0 & 99 & 105.1(156.0) & 46.0 & 87 & 20.1 & 0.301 & 0.223 \\
\hline SA frequency & $9.36(9.43)$ & 7.0 & 99 & $8.06(6.02)$ & 7.0 & 87 & 1.3 & 0.211 & 0.030 \\
\hline Percentage of LTSA ${ }^{*}$ & $35.1 \%$ & & 46 & $25.3 \%$ & & 25 & $9.8 \%$ & 0.023 & 0.017 \\
\hline \multicolumn{10}{|l|}{ 5-years follow-up } \\
\hline Total SA duration \pm & $166.3(202.7)$ & 93.5 & 94 & $113.5(175.2)$ & 58.4 & 78 & 52.8 & 0.034 & 0.035 \\
\hline SA frequency & $11.44(11.18)$ & 9.0 & 94 & $9.91(7.14)$ & 8.0 & 78 & 1.53 & 0.245 & 0.059 \\
\hline Percentage of LTSA* & $35.9 \%$ & & 47 & $22.2 \%$ & & 22 & $13.7 \%$ & 0.009 & 0.004 \\
\hline
\end{tabular}

${ }^{1}$ Crude analysis using Poisson regression without adjustments for covariates.

${ }^{2}$ Adjusted analysis using Poisson regression for covariates; age, gender, job function and long-term illness.

\pm Total SA duration including $>28$ days SA *Percentage LTSA is calculated annually 


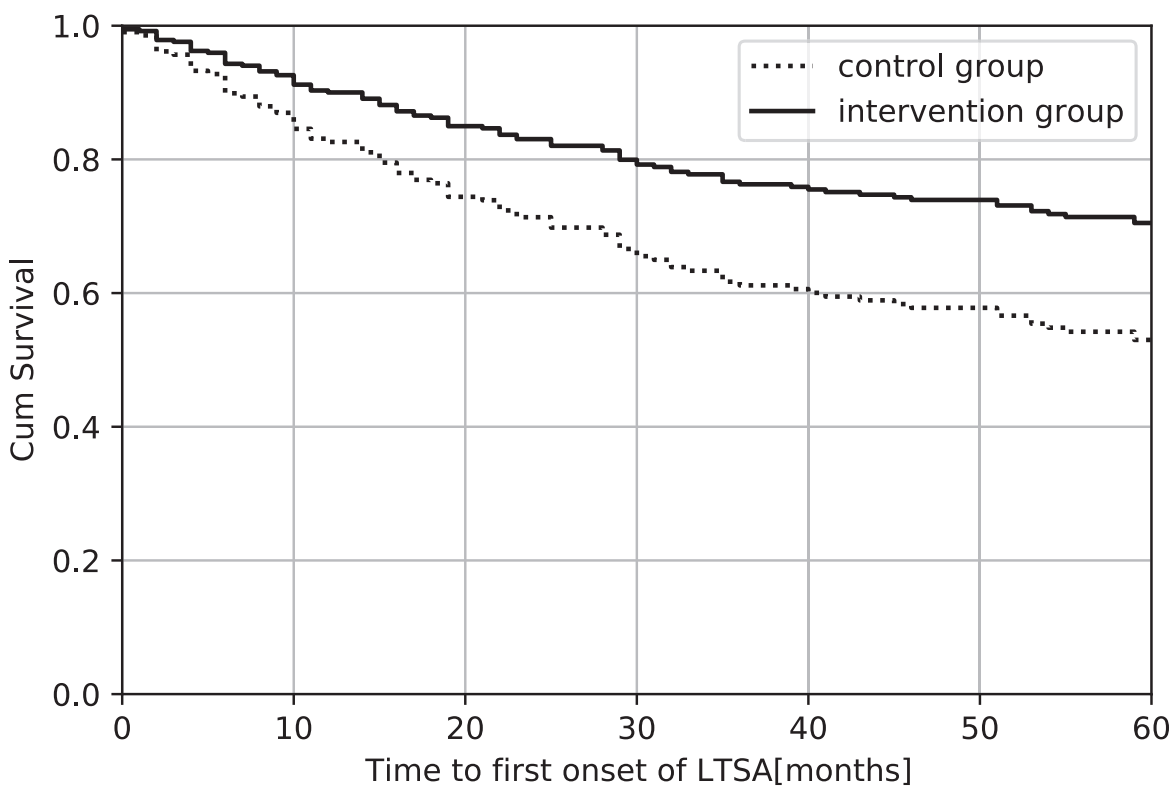

Figure S1: Time till first onset of LTSA in months (RCT I per protocol analysis) (HR 0.53; 0.34-0.83)

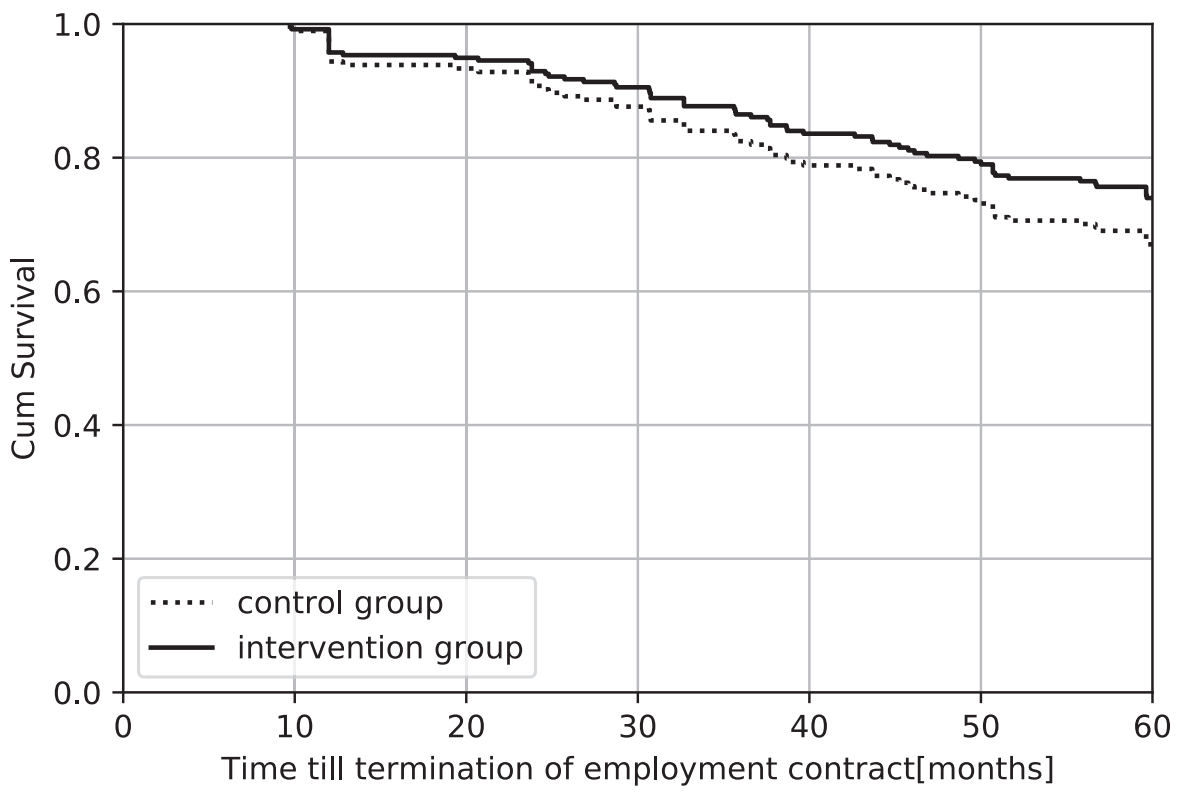

Figure S2: Time till termination of the employment contract with the company in months (RCT I per protocol analysis) (HR 0.78; 0.47-1.30). 
Table S2: Overview efficacy of the indicated prevention strategy on SA parameters for RCT II (per protocol analysis)

\begin{tabular}{|c|c|c|c|c|c|c|c|c|c|}
\hline & \multicolumn{3}{|c|}{ Control group } & \multicolumn{3}{|c|}{ Intervention group } & \multirow{2}{*}{$\begin{array}{c}\text { Mean } \\
\text { Difference }\end{array}$} & \multirow[t]{2}{*}{ P-value ${ }^{1}$} & \multirow[t]{2}{*}{ P-value ${ }^{2}$} \\
\hline & Mean (SD) & Median & $\mathrm{N}$ & Mean (SD) & Median & $\mathrm{N}$ & & & \\
\hline \multicolumn{10}{|l|}{ 2-years follow-up } \\
\hline Total SA duration \pm & $81.2(114.2)$ & 29.5 & 56 & $48.8(70.5)$ & 22.0 & 43 & 32.3 & 0.056 & 0.079 \\
\hline SA frequency & $3.68(2.88)$ & 3.0 & 56 & $3.12(2.46)$ & 3.0 & 43 & 0.56 & 0.300 & 0.343 \\
\hline Percentage of LTSA* & $18.6 \%$ & & 13 & $19.6 \%$ & & 9 & $-1 \%$ & 0.772 & 0.754 \\
\hline \multicolumn{10}{|l|}{ 3-years follow-up } \\
\hline Total SA duration \pm & $106.6(135.8)$ & 43.5 & 50 & $93.5(119.3)$ & 34.0 & 40 & 13.1 & 0.595 & 0.560 \\
\hline SA frequency & $5.46(4.04)$ & 4.5 & 50 & $4.62(3.53)$ & 4.0 & 40 & 0.84 & 0.284 & 0.220 \\
\hline Percentage of LTSA* & $21.4 \%$ & & 15 & $32.6 \%$ & & 15 & $-11.2 \%$ & 0.479 & 0.538 \\
\hline \multicolumn{10}{|l|}{ 4-years follow-up } \\
\hline Total SA duration \pm & $134.0(185.2)$ & 36.5 & 40 & $124.7(179.8)$ & 35.0 & 39 & 9.3 & 0.796 & 0.688 \\
\hline SA frequency & $6.45(5.42)$ & 5.0 & 40 & $5.92(4.05)$ & 5.0 & 39 & 0.53 & 0.599 & 0.591 \\
\hline Percentage of LTSA* & $17.1 \%$ & & 12 & $30.4 \%$ & & 14 & $-13.3 \%$ & 0.598 & 0.747 \\
\hline \multicolumn{10}{|l|}{ 5-years follow-up } \\
\hline Total SA duration \pm & $170.5(249.0)$ & 54.1 & 33 & $184.8(251.4)$ & 91.0 & 33 & -14.3 & 0.794 & 0.869 \\
\hline SA frequency & $7.15(5.97)$ & 6.0 & 33 & $7.03(5.06)$ & 6.0 & 33 & 0.12 & 0.923 & 0.869 \\
\hline Percentage of LTSA* & $17.1 \%$ & & 12 & $30.4 \%$ & & 14 & $-13.3 \%$ & 0.652 & 0.748 \\
\hline
\end{tabular}

${ }^{1}$ Crude analysis using Poisson regression without adjustments for covariates.

${ }^{2}$ Adjusted analysis using Poisson regression for covariates; age, gender, education level and long-term illness. .

\pm Total SA duration including $>28$ days SA *Percentage LTSA is calculated annually 


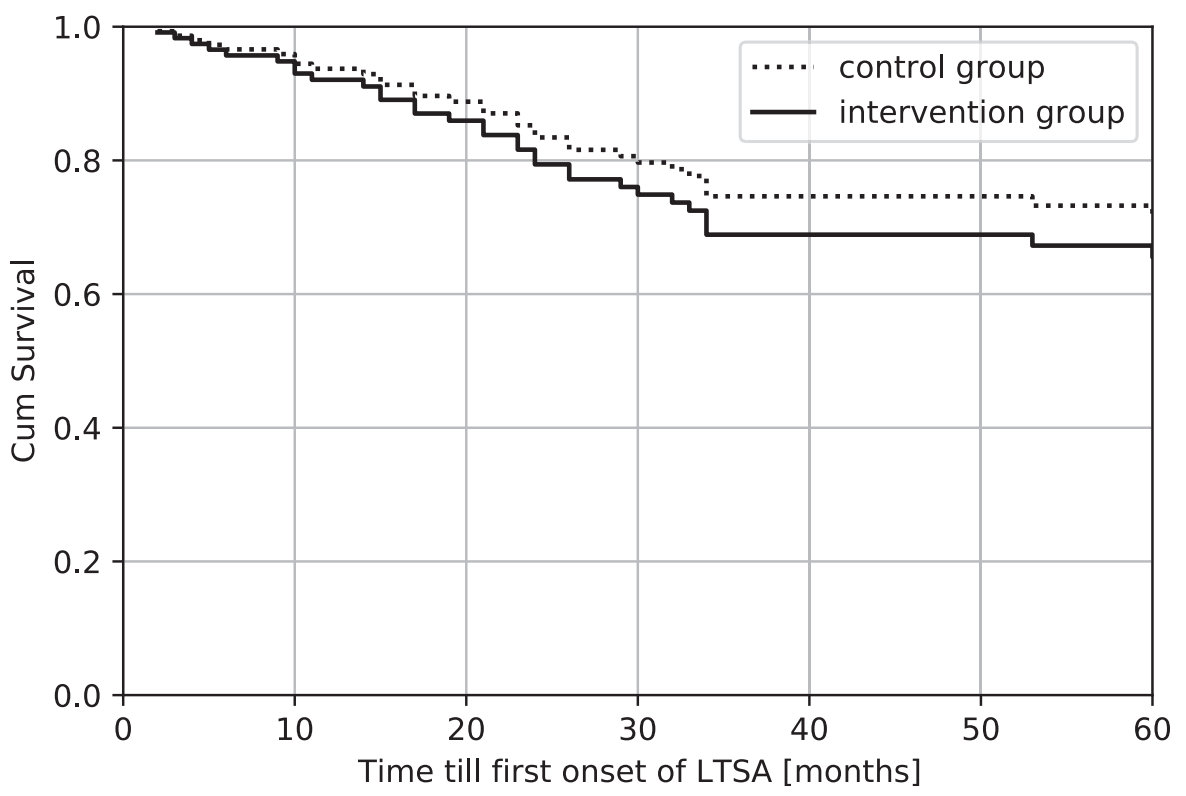

Figure S3: Time till first spell of LTSA (RCT II per protocol analysis) (HR 1.27; 0.63-2.56)

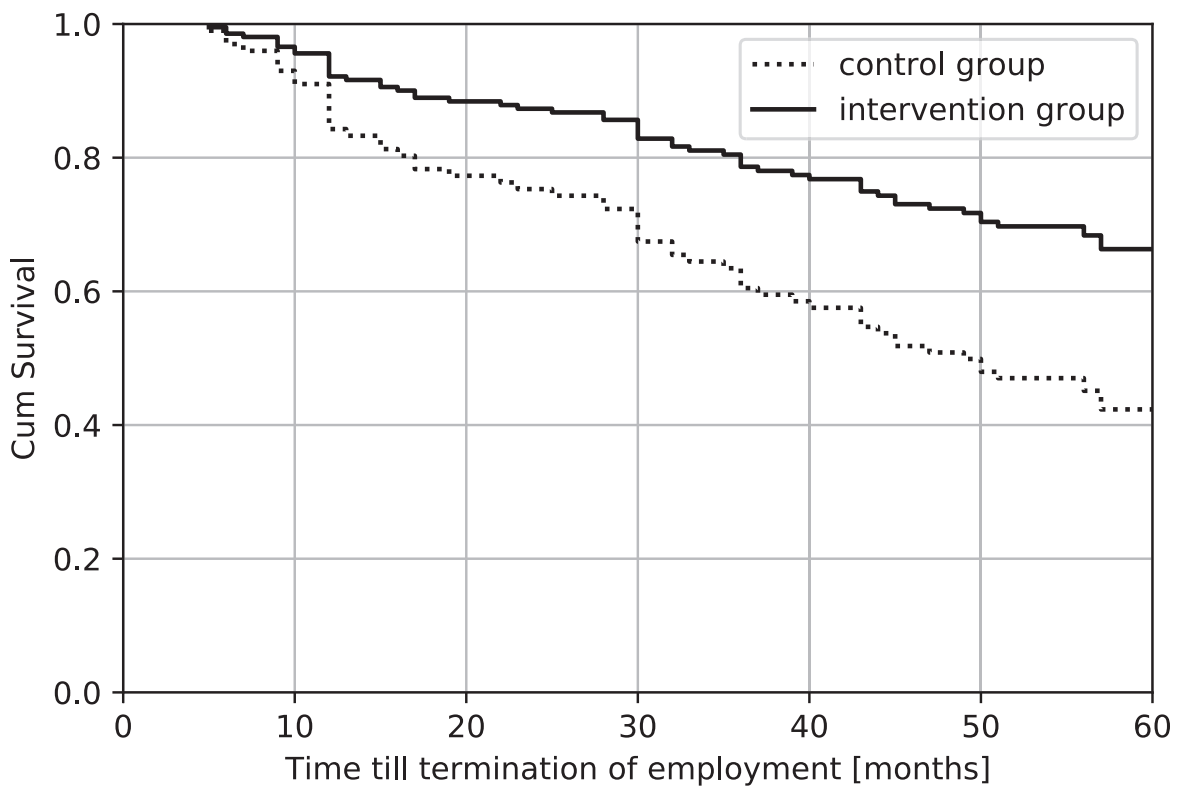

Figure S4: Time till termination of the employment contract with the company in months (RCT II per protocol analysis) (HR 0.48; $0.27-0.85$ ) 



\section{Chapter 5}

The impact of an indicated prevention strategy on sick ass absence spells accord ng to their diagnostic labels and anumbutions

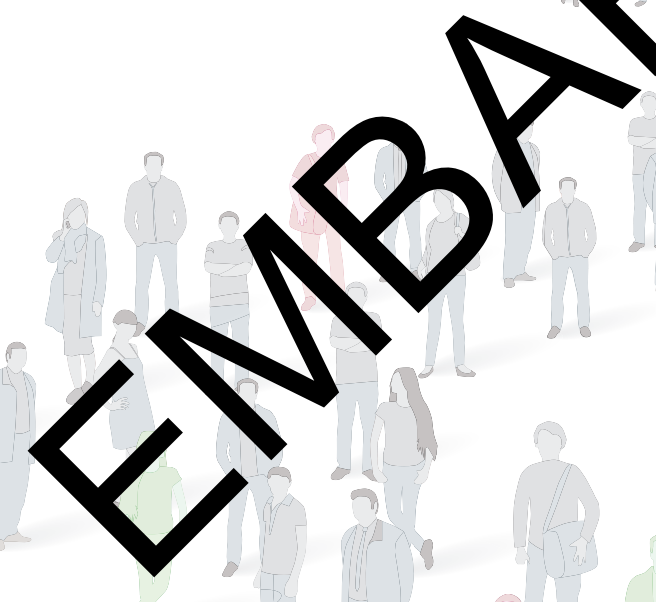

Sophie H Klasen, MSc, Ludovic GPM van Amelsvoort, PhD, Nicole WH Jansen, PhD, Jos JM Slangen, IJmert Kant, PhD

CAPHRI School for Public Health and Primary Care, Department of Epidemiology, Faculty of Health, Medicine and Life Sciences, Maastricht University, Maastricht, The Netherlands. 


\section{Chapter 6}

\section{Exploring expected and perceived facilitators and barriers of an indicated prevention strategy to prevent future long-term sickness absence. A qualitative study among employers and employees}

Sophie H Klasen, MSc, ${ }^{1}$ Ludovic GPM van Amelsvoort, PhD, ${ }^{1}$ Inge Houkes, $\mathrm{PhD}^{2}$ Nicole WH Jansen, $\mathrm{PhD},{ }^{1}$ IJmert Kant, $\mathrm{PhD}^{1}$

${ }^{1}$ CAPHRI School for Public Health and Primary Care, Department of Epidemiology, Faculty of Health, Medicine and Life Sciences, Maastricht University, Maastricht, The Netherlands.

${ }^{2}$ Department of Social Medicine, Faculty of Health, Medicine and Life Sciences, Maastricht University, Maastricht, The Netherlands. 


\section{Abstract}

\section{Objective}

An indicated prevention strategy (IPS), consisting of a screening questionnaire and early treatment, was found to be effective for the prevention of future long-term sickness absence (LTSA) in two large Dutch RCT's. This IPS aims to detect employees who have a high risk to become absent, and subsequently offer them early treatment. Despite the overall effectiveness, only a few companies have implemented this strategy so far. This suggests that companies may not be convinced of the (cost) effectiveness of this strategy yet. In companies where IPS has been implemented, screenings uptake and adherence to early treatment appeared to be moderate, indicating that both employees and employers might perceive barriers.

\section{Methods}

The aim of this qualitative study was to explore the expected and perceived facilitators and barriers for the implementation of the IPS. Semi-structured interviews were conducted with 9 employers and 11 employees (acquainted and unacquainted with IPS) from large companies. Purposive sampling was used to recruit participants. All interviews were transcribed and analyzed thematically.

\section{Results}

The employers believed they were primarily responsible for psychological and workrelated health complaints and SA, while the employees felt responsible for health complaints related to their lifestyle. According to the employees, the responsibility of the employer was solely related to work-related health. This finding exposed a relation with the health culture, which was solely based on creating a safe work environment, omitting psychological health issues. The efficacy of this IPS regarding reducing SA was estimated positive, however, the efficacy regarding LTSA was questioned. Fear of a privacy breach was often mentioned by the respondents as an important barrier.

\section{Conclusion}

This study showed that the health culture within a company may be important for the perceived responsibility towards SA and health. A health culture which primarily focuses on physical complaints may raise barriers for the adoption and implementation of this preventive strategy. Participants' perceptions of the nature of LTSA and the fact that not all participants were familiar with the exact content and phasing of IPS may have doubted the efficacy regarding LTSA. This study provides important clues for future and improved implementation of IPS. 


\section{Background}

Long-term sickness absence (LTSA) is a major occupational and public health issue in Western countries [1]. In the Netherlands, employers generally are obliged to pay at least $70 \%$ of the employee's salary in the first two years of sickness absence (SA) (regardless the cause), often resulting in high costs for employers [2-4]. Returning to work after a period of LTSA is often difficult and may even lead to permanent work disability, affecting employee wellbeing and resulting in further costs for employers and society [5-8]. SA is a complex, multi-factorial phenomenon, which often has personal, work-related, and social determinants [9] [10]. For the prevention of SA, return to work strategies to prevent SA, as well interventions based on principles of universal or selective prevention (which only focus on one or two explaining factors) appear to be less effective [8, 11, 12]. Because often due to the multifactorial etiology of SA, a more comprehensive approach is needed $[11,12]$. An indicated prevention strategy might be more promising in the prevention of LTSA since it focuses on multiple factors concerning SA. Indicated prevention strategies are designed to prevent the onset of a disease or health issues, the individual is not yet sick but shows early warning signs and is, therefore 'at high-risk' [13]. Innovative indicated prevention strategies have been developed, which showed their efficacy in predicting and preventing future SA $[12,14,15]$. This study will focus on one of these strategies, henceforward called 'IPS'. The IPS consists of a screening questionnaire, followed by early consultation with the occupational health professional (OHP) for employees at high risk for future LTSA. Following the consultation, early treatment starts with the OHP or another health professional [16]. This IPS has been evaluated in two large Dutch RCTs and appears to be effective concerning the prediction and prevention of future SA and improving the mental health status of employees $[12,14,16,17]$.

Although the potential benefits of these strategies are high, their application/ implementation is still rather low [5]. This suggests that employers/companies are not convinced of the (cost) effectiveness of this IPS yet. Moreover, in the companies where IPS has been implemented, screenings uptake and participation in the early intervention is only moderate, suggesting that employees may encounter barriers or hindering factors with respect to participation in the IPS [18]. Many different stakeholders with different interests are involved in workplace health and disability prevention programs, including this IPS. This may complicate the adoption and implementation process of such programs [19-21]. The IPS involves (i) the employer who organizes and bears the cost of screening all of the employees and early interventions for employees identified to be at risk for future $\mathrm{SA}$, (ii) employees who are invited to fill in the screening questionnaire, (iii) employees identified as being at high risk for future SA who are invited for early intervention, and (iv) the OHP who will provide consultation and early intervention. These stakeholders all have 
different interests regarding the prevention of LTSA, and thus the perceived facilitators and barriers will probably differ, which may inhibit the adoption and implementation of the strategy. Therefore, it is very important to explore the perceived and experienced facilitators and barriers of adoption and implementation of this strategy.

Earlier studies into general, universal, or selective occupational health prevention have investigated relevant facilitators and barriers, which could also apply to this IPS. Improving the productivity and well-being of employees, reducing healthcare costs, and indirect costs related to absenteeism and permanent disability were mentioned as important facilitators by employers [22] [23]. Employees expected improvements in physical as well as psychological health and improvements in their general well-being [22, 24]. Relevant barriers for employers are the high cost of the screening and intervention, logistical issues, and time scarcity [25]. Barriers according to the employees were related to privacy issues and fear of discrimination or stigmatization at work [26]. The early character of this intervention, before employees, actually report sick, and the primary focus on high-risk employees for future LTSA may reveal different facilitators and barriers. A comparable study showed with the use of a survey that questions concerning risk perceptions regarding diabetes, cardiovascular disease and chronic kidney disease created misconceptions. The high-risk classification may create difficulties, while employees may be unaware of the true meaning of being at high-risk [27].

The perspective of the occupational physician (OP) of this IPS has been studied earlier. This study by de Brouwer et al. 2017 showed that important barriers from the perspective of OPs were amongst others; communication skills, appropriate training for the OHP and privacy issues related to labeling of high risk employees [28]. The current study will therefore only explore the views from the employers and employees.

It is expected that acquaintance and/or experience with this IPS could also affect the facilitators and barriers, and hence on its adoption and implementation in organizations. Acquaintance with this IPS could resolve the initial concerns related to a preventive intervention or shed light on other important issues which were not foreseen. To our knowledge, no earlier research has investigated whether acquaintance with a preventive strategy will affect how the barriers and facilitators are perceived.

This study aims to explore potential facilitators or barriers regarding this IPS, from the perspectives of employers and employees acquainted/unacquainted with the strategy. We believe that beliefs about these facilitators and barriers are determined by the employers/ employee's perceptions, norms, and values, this study therefore requires a qualitative approach. Our main research question is: What are the facilitators/barriers of an indicated prevention strategy preventing future LTSA according employers and employees who are acquainted or unacquainted with the IPS? 


\section{Methods}

In order to explore the expected and perceived facilitators and barriers of an indicated prevention strategy (IPS), a qualitative design was chosen. We performed semi-structured interviews with employers and employees. The IPS context under study consisted of a screening phase, using a screening questionnaire and a subsequent intervention for employees identified by the screening phase as being at high risk for future LTSA.

\section{Screening questionnaire}

The screening questionnaire, Balansmeter, includes 34 predictors, each measured by multiple-choice or dichotomous questions. The questions cover different domains: work environment (e.g. working conditions, psychological job demands), characteristics of the private situation, (mental) health status, demographic factors, and SA history. Using an algorithm based on the weighted factors of the individual items of the model, a total score can be calculated, with higher scores indicating a higher risk for future LTSA [14]. A pre-determined cut-off point is used to identify high-risk employees. Different versions have been developed for office and industry workers, with separate algorithms for male and female employees [14]. Screening data generated by this questionnaire are part of an employee's medical file and as prescribed by Dutch law, may not be shared with the employer, except with the worker's explicit consent. In the daily practice of the companies that have already implemented the preventive strategy, employees are invited to participate every three years. The three year frequency has not been validated in earlier studies, however this is in line with the frequency of occupational prevention interventions.

\section{Early consultation and intervention}

Employees at high risk according to the screening questionnaire are invited for an extensive, one-to-one consultation with an occupational health professional (OHP). The OHP was chosen as the expert for consulting with high-risk employees because $s /$ he is specifically trained to recognize work-related and non-work-related conditions and their interactions. The structured early consultation involves several steps, during which the results of the screening questionnaire will be discussed and a broad range of additional anamneses can be performed to consider options for treatment or guidance. A special training is available for the OHPs to facilitate working with the preventive questionnaire, but this training is not obligatory [28]. This consult may then result in a targeted intervention focusing on the specific complaints presented by the employee. The targeted intervention may consist of various conventional treatments, ranging from additional socio-medical counseling by the occupational physician to psychotherapy, counseling by a social worker, or specialized and/ or intensified care for a specific disease. A graphic overview of the IPS is shown in Figure 1. 
(1) Screening questionnaire 'Balansmeter'

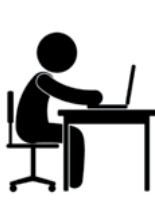

2) Consultation with OHP for high risk employees
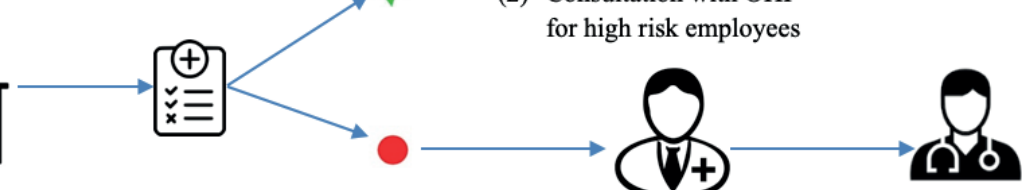

High risk for future long-term sickness absence

(3) Early treatment with OHP or other professional

Fig. 1 Indicated prevention strategy; prediction and early consultation to prevent future LTSA

\section{Study design, sample and procedure}

We employed purposive sampling to recruit employers and employees of varying age (almost all 35-65) and acquainted/not acquainted with the IPS. All employees worked for the same two sites of a petrochemist, this petrochemist has multiple departments were some had implemented this strategy in the past and others had not. The employers were chosen from a university, a Dutch bank, a consultancy company, and a petrochemist (same as the employees). Based on the job levels, we expect the education level of the employees to vary from low to high. The job functions of the employers presume a high educational level.

Employees were considered acquainted with this IPS when they had filled in the screening questionnaire of which some were additionally invited by the OHP for early treatment. Employees were considered unacquainted with this strategy when they had only heard of this strategy and did not fill in the screening questionnaire. The employers were considered to be acquainted with this IPS when they had used the intervention themselves or had a lot of knowledge about this IPS. Employers were seen as unacquainted with this IPS when they knew only basic information about this strategy. Especially the beliefs of the unacquainted employees and employers were considered very important to obtain insights into potential reservations regarding this strategy. For privacy reasons, we could not make a further distinction in acquaintance with this IPS. For the same reason, the researchers did not know whether respondents had been at high or low risk in the past for LTSA. We aimed to include large companies in this study, while this IPS has so far only been implemented in large companies (>10.000 employees). Investigating the perspectives of similarly large organizations, as the views of employers and employees from Small and Medium-sized Enterprises (SMEs) might be different due to different contexts, structures, cultures and quality of occupational healthcare. Employees and employers were chosen from different large institutions, acquainted and unacquainted with the IPS. An overview 
of the respondents is shown in Table 1 . All respondents were invited for the interview via email, in which the aim of the study was explained.

Table 1: Characteristics of the study subjects

\begin{tabular}{rcccc}
\hline & Stakeholder & Gender & Company & Acquaintance with IPS \\
\hline 1 & Employer & Female & Bank & Yes \\
2 & Employer & Female & Bank & Yes \\
3 & Employer & Female & Consultancy & Yes \\
4 & Employer & Male & Petrochemist & Yes \\
5 & Employer & Male & University & No \\
6 & Employer & Male & University & No \\
7 & Employer & Male & Petrochemist & No \\
8 & Employer & Male & Petrochemist & No \\
9 & Employer & Female & Petrochemist & No \\
10 & Employee & Male & Petrochemist & yes \\
11 & Employee & Male & Petrochemist & yes \\
12 & Employee & Male & Petrochemist & yes \\
13 & Employee & Male & Petrochemist & yes \\
14 & Employee & Male & Petrochemist & yes \\
15 & Employee & Male & Petrochemist & yes \\
16 & Employee & Male & Petrochemist & yes \\
17 & Employee & Male & Petrochemist & No \\
18 & Employee & Male & Petrochemist & No \\
19 & Employee & Male & Petrochemist & No \\
20 & Employee & Male & Petrochemist & No \\
\hline
\end{tabular}

\section{Data collection}

All respondents were invited for a face-to-face interview which took place between June 2017 and March 2018. All interviews were conducted in a private room at the respondents' workplace and were conducted in Dutch by the first author (SK). A topic list was used for each interview (Additional file 1). The topic list gave the first author a starting point, and according to their answers, it was decided how many more questions needed to be asked about a certain topic to get a good idea of the perception. The topic list was used to obtain a more in-depth discussion. The topics were derived from the relevant literature with a focus on the values, barriers, and facilitators of preventive interventions [23, 25, 26]. Core topics, like the expected effect of this IPS on LTSA, the effect on multiple facets of health, and the level of trust in this strategy, were similar for all stakeholders. Stakeholder-specific 
topics were added, e.g. costs of the strategy for the employers and fear of a privacy breach for the employees, to be able to evaluate more stakeholder-specific views.

Prior to each interview, the respondents were informed by the researcher by e-mail about the purpose of this study followed by a summary of the IPS and its results in the past. Before the start of each interview, the IPS was again explained by the interviewer, to provide the respondents with basic knowledge about the strategy. It was stated that all information given would be treated confidentially. Verbal informed consent was requested before each interview, and the ethical clearance is provided by FHML REC from Maastricht University (FHML-REC/2019/020). The interviews were taped and lasted 40 minutes on average. They were digitally recorded and transcribed verbatim by an independent assistant. The transcribed interviews were all in Dutch, but the quotes used for this article were translated into English. Data saturation was reached, after approximately seven interviews with the employees and six interviews with the employers, meaning that no new themes or sub-themes emerged from the data.

\section{Data analysis}

The interviews were analyzed thematically, which resulted in main themes with further sub-categorization [29]. This approach enables deductive reasoning while also permitting new themes to emerge from the data. One researcher coded the interviews, and three researchers (IJK, LVA, IH) assessed the coding phase. The coding labels were discussed and adjusted, or coding themes were added with the help of the same researchers who acted as peer reviewers (IJK, LVA, IH). Additional file 2 gives an overview of all the themes with their corresponding codes. Nvivo version 11 was used for the data analysis.

\section{Results}

Three main themes emerged from the data: 'Values', 'Facilitators', and 'Side effects and barriers', each with its sub-themes. To give a clear overview, we categorized the main themes and sub-themes in Table 2. It appeared that the interviewees' ideas about responsibility for SA and health and the presence of a health culture were important underlying values of perceived barriers and facilitators of the IPS. For the facilitators, we focused specifically on the benefits this strategy could provide for SA, future LTSA, and health.

We distinguished between the expected and perceived side effects for employers and employees. The expected/perceived side effects were related to costs for both the employer and the employee, but in a different manner and concerning the effects on healthcare use. A wide variety of barriers was mentioned which were further divided in adoption barriers 
Table 2: Main themes and sub-themes items for employers and employees

\begin{tabular}{ll}
\hline Main themes & Sub-themes \\
\hline Values & Responsibility \\
- Perceived responsibility for SA & - Perceived responsibility for health \\
Health culture & - Presence of a health culture \\
\hline Expected and perceived positive effects \\
- Effect of IPS on future SA \\
- Effect of IPS on future LTSA \\
- Effect of IPS on health \\
Expected and perceived side effects employer \\
- Costs (for the IPS) \\
- ROI \\
- Costs and benefit balance \\
- Healthcare costs \\
Expected and perceived side effects employee \\
- Costs (own payment for preventive care) \\
- Restraint own payment for use of preventive care \\
- Attitude towards employer if he pays for preventive care \\
Adoption barriers \\
- Trust in the privacy of information \\
- Confidence in the IPS \\
- Discrimination/stigmatization \\
Practical implementation barriers \\
- Insufficient time to fill out the questionnaire \\
- Communication issues with the IPS \\
- Low -frequency of the IPS \\
\hline
\end{tabular}

and barriers which could obstruct the implementation of the IPS. Adoption barriers were often related to privacy issues, confidence in the strategy, and fear of discrimination. The implementation barriers were more pragmatic, like insufficient time to fill out the questionnaire, communication issues with the IPS, and the low-frequency of the IPS. The narrative is organized according to this division of main themes and sub-themes.

\section{Values}

Perceived responsibility for $S A$

All employers independent of acquaintance/unacquaintance perceived the reduction of SA as a major responsibility of theirs and many agreed it was also the responsibility of the employee. Employers did not feel responsible for issues such as the flu, but mainly 
for work-related or psychological issues. [Employer8] "I do not mean flu or something like that, but really when it comes to psychological complaints, then I feel clear responsibility." One unacquainted employer mentioned feeling almost 100\% responsible for SA related to psychological issues.

All employees regardless their level of acquaintance with the IPS, felt there was a shared responsibility for SA with their employer. [Employee18] "Yes, if it has to do with my own lifestyle I do not think so, but if it has to do with the absenteeism due to the nature of work or the substances that are present at the worksite, then I think it's the employer's responsibility." The employer was held responsible if SA was related to work, the work environment, or work-related stress. The employees felt it was their responsibility if SA was a result of their lifestyle.

\section{Perceived responsibility for health}

Most of the employers regardless their level of acquaintance with the IPS, believed that health is a shared responsibility with their employees. Often, they felt responsible when the health complaints were work-related, like stress or pressure from work tasks. [Employer7] "I am responsible that the employee can do his work well and we also have a role in the long-term effects of stress and work pressure." If bad health was a result of lifestyle choices, they did not feel this responsibility, and only a few felt the need to interfere with lifestyle choices, when the latter negatively affected the employee's work.

Many employees regardless their level of acquaintance with the IPS, believed the responsibility for their health was as much their responsibility as their employer's. According to them, the employer was responsible for creating a safe work environment, and the employees were responsible for having a healthy lifestyle. [Employee20] "Fiftyfifty; As an employer, you are responsible for creating a safe working environment and ensuring that people feel comfortable in their working environment. The employee has a great responsibility to keep himself healthy and vital to be fit for his purpose." In comparison with employees who were unacquainted with the strategy, some employees acquainted with the strategy, thought the employer was less responsible for their health.

\section{Health culture}

According to all employers acquainted with the strategy, health was embedded in the culture of the organization, with attention being paid to psychological issues, mindfulness sessions, and healthy living workshops and programs. Many employers unacquainted with the strategy mentioned the absence of a health culture. Some mentioned that the health culture was related to safety, which was the primary focus for them. [Employer9] "Safety is our number one priority, but this is not the case for health, while health actually also belongs to safety." For them, a health culture was established by creating a safe place 
to work where they also focused on sports and healthy eating programs. Here countering psychological issues to improve the health situation of their employees is not part of the health culture.

Almost all employees regardless their level of acquaintance with the IPS, associated a health culture with safety, healthy eating, and sports programs. They often felt the organization did not meet their expectations to improve their health situation. Some employees unacquainted with the strategy mentioned that the health culture was focused on physical health which was related to their work and not on the health complaints outside work. [Employee20] "Focus is very much on physical health and preventing damage during working hours and less on long-term, not work-related aspects." Attention to their psychological complaints did not seem to be embedded in the health culture.

\section{Facilitators}

\section{IPS effect on future SA}

Some of the employers acquainted with the strategy believed this IPS would decrease SA, however, others thought this decrease would be limited due to only a small amount of high-risk employees. The employers unacquainted with the strategy often believed in the preventive effect of the strategy and the benefit of insight in the current health state. [Employer3] "So yes, in that sense it is preventive, whether we prevent dropout... I do not know. I find it complicated whether we might find people earlier. It is, of course, the idea.". Most employers agreed that the effects of the IPS on SA will be different for people from different departments in the organization. [Employer3] "I believe there is a difference in SA between the different employees. For example, our staff has much higher absenteeism than Consultancy." The difference was often explained by different work stressors.

All employees unacquainted with the strategy expected a decrease in SA. [Employee13] "I think if you are actively engaged, the absenteeism will go down." The awareness about their health situation was also mentioned by the employees as an important factor which could trigger them to cope with their health problems at an early stage. The employees acquainted with the strategy were divided in the expected efficacy of this IPS on SA, some believed it would decrease SA others thought the strategy was without any obligation and dependent on the type of person or work situation. [Employee11] "So the advantage of doing office work is that you have less chance to get back/knee/joint complaints in comparison to the work outside, which is usually in cramped positions, or heavy lifting ... that is the difference." Both employers and employees expected a different effect of this IPS on SA for employees in different departments often dependent on the type of work. 


\section{IPS effect on future LTSA}

All employers acquainted with the strategy had positive expectations about the IPS efficacy on LTSA, however, often the once every three-year frequency was mentioned as inadequate to truly capture LTSA. They also felt that the IPS could not be the only strategy for the prevention of LTSA. [Employer3] "I am feeling confident about the preventive strategy as part of something larger. I think if you do it alone, well maybe, I do not know, for me it is more than that. Also to make it alive." Some employers unacquainted with the strategy mentioned the efficacy of this IPS was also dependent on the cause of SA. The efficacy was questioned by one due to the belief that LTSA was mostly related to cancer or other serious health conditions. Another employer added that LTSA is caused by psychological as well as physical complaints and therefore a more holistic strategy is needed than solely this IPS.

Employees had varying opinions about the possible effects of the IPS on LTSA independent of acquaintance/unacquaintance with the strategy. Some thought it would be effective in the long-term, while others had different reasons to believe that IPS would not be effective in decreasing LTSA. Some employee associated physical complaints primarily with the cause of LTSA and therefore the efficacy of this IPS would be less. [Employee15] "I think that you select the people who have periods of short-term SA. There may be two or three people who are mentally ill for a long time, but most people who are ill for a long time have physical complaints or health complaints directly." In addition, we noticed that some employees, regardless their level of acquaintance with the IPS, were apparently unaware of the OHP consultation being part of the IPS. [Employee10] "Then it is not just filling in that paper, but then you get the feeling that you are actually being looked at medically." The OHP consultation is an essential part of the IPS because it makes sure the employees receive early treatment when they get a high score on the Balansmeter, which means a high risk for future LTSA. This unawareness of the OHP consultation could have affected the views of the employees on the effectiveness of IPS on LTSA.

\section{IPS effect on health}

Many employers independent of acquaintance/unacquaintance with the strategy believed in a positive effect of the IPS on health through greater awareness about the health situation of employees. Some employers acquainted with the strategy expected a larger effect on psychological health rather than physical health. [Employer4] "My estimation would be that the impact on psychological symptoms is greater than on the physical symptoms." Some employers unacquainted with the strategy were more concerned by the subsequence of the screening questionnaire and the possible overestimation employees could make about their health.

Employees who were acquainted with the IPS were almost all positive about the 
potential effects on health, but for different reasons. For some employees, their health would benefit through lifestyle advice, for others it was related to an improvement in psychological health. [Employee10] "And if your diet is good and you feel good and you are physically right, SA will also be less." [Employee15] "That the psychological symptoms will be picked out and you could do something with that, then I think you can really benefit from it." Some employees who were unacquainted with the IPS were less positive about the effects on health. Often they focused only on the screening questionnaire and forgot the early consultation. [Employee18] "Filling in a checklist does not affect my health." The consultation with the OHP is considered to be a prerequisite for health improvement but is often not a visible part of the IPS for the employees who are not identified as being at high risk for future LTSA and invited for an early consultation.

\section{Side effects \& barriers}

\section{Employer views}

Almost all acquainted employers were unaware of the costs of this IPS. However, independent of acquaintance/unacquaintance the costs of this IPS were not considered an important factor in the decisions for implementation by many employers. The focus was less on costs and more on the social revenue resulting from being a good employer. [Employer3] "I think it's worth the investment from several perspectives. It can of course work preventively, but it is also a bit of awareness, so also good employment practices." The employers had different opinions about the Return on Investment (ROI). The ROI of an intervention was not seen as important by everyone; it becomes more important if the costs of an intervention are higher.

Most employers regardless their level of acquaintance with the IPS, expected and often favored a short-term increase in general healthcare use followed by a long-term decrease in healthcare use. They expected more efficient or tailored care for their employees. [Employer2] "I expect less health care use. At least, more efficient use of care. That people do not wait too long but rather ask for help." Few employers unacquainted with the strategy mentioned that this was dependent on the health subject and on the way, employees were confronted with their health status. None of the employers perceived the expected increase in healthcare use in the beginning or stability in healthcare use, before and after implementation as a barrier.

\section{Employee views}

Almost all employees who were acquainted with the IPS did not perceive a payment for preventive care as something negative; according to them, this care was inevitable. In the Netherlands, care is paid from your obligatory deductible excess, therefore it is 
expected that people are used to paying the initial part of their healthcare. The employees mentioned that it would be better to receive care at an early stage, to protect their health and prevent higher costs. [Employee16] "If my health is getting worse, I will have to pay at any given time. It's for your own health, come on!" Employees who were unacquainted with IPS had a different perception of the costs of preventive care. The employer was expected to pay for preventive care if it was related to work issues; the employee was expected to pay for health issues not related to work. The deductible for preventive care was definitely not seen as a restraint to use care by most employees acquainted with IPS. They thought their salary was high enough to pay for the care needed. High costs for preventive care were mentioned as a barrier by some of the employees unacquainted with IPS, mainly that their salary would not be high enough to afford preventive care. Almost all employees agreed that it would be positive if the employer would pay for the preventive care but often added that it would never happen if the costs were too high. Few mentioned that they would only agree to this if it was work-related and not self-inflicted. [Employee13] "If I break my leg on a football field because I'm playing football, then I believe I have to pay for it myself." A division is clearly made by the employees if SA is a result of their private actions or work-related.

Some employees acquainted with the IPS expected less healthcare due to preventive actions, which could also result in less use of acute healthcare. [Employee16] "I think less. If someone starts to get sick, sickness can be tackled early." Other employees acquainted with IPS mentioned that it would depend on the type of person or how they would interpret the advice from the OHP. The employees unacquainted with IPS all expected more healthcare use. Almost all employees believed that more people would use preventive care if the employer paid for it. Few mentioned that this would not make a difference or that it was dependent on the type of person.

\section{Barriers related to adoption}

Few employers independent of acquaintance/unacquaintance with the IPS mentioned possible privacy concerns from employees as an issue and the current privacy debate which could result in employees not wanting to share their personal information. Some employers unacquainted with the IPS mentioned the importance of persuading the line managers to stimulate the adoption. By some, the need for positive framing of the strategy was mentioned to support the adoption of this strategy by the employees. [Employer6] "Currently it has a negative feeling; we need to decrease SA. Turn it around; we have a fun instrument which will benefit your work process, health and create a good work environment". Some unacquainted employers mentioned the importance of a validated questionnaire. The employers mentioned different barriers that could inhibit the adoption process of the IPS. 
Often employees independent of acquaintance/unacquaintance with IPS mentioned privacy as the biggest barrier to filling out the questionnaire. [Employee15] "I think there are a lot of people who may be hesitant or afraid that it is not confidential." The interviewees had no problems filling out the screening questionnaire but assumed that other people might have, as they may be afraid the information would be made available to the employer. [Employee20] "So it really has to be a doctor-patient relationship. Fully confidential." General privacy breaches were also mentioned as examples that nothing is confidential nowadays. All acquainted employees believed their privacy was protected when they participated in the IPS. A few mentioned being reassured that the physician practices doctor-patient confidentiality and that a university was involved, which also increased the trustworthiness. Some added that there was a possibility the employees would not be completely honest when filling out the screening questionnaire if they were scared of privacy breaches or the employer finding out. The unacquainted employees could not judge the confidentiality of the IPS, because they felt they did not have enough information. However, the protection of personal information was mentioned as highly important.

Almost all employers regardless their level of acquaintance with the IPS, had confidence in the effectiveness of the IPS, but often they added a side note. Some employers acquainted with IPS mentioned that the strategy was only effective if a lot of employees participated or that the strategy needed to be part of something bigger to prevent LTSA. [Employer1] "In principle a lot, if there are enough people who cooperate with the IPS I think it can have an effect on the absenteeism figures." A few employers unacquainted with the IPS mentioned they were positive but would like to have more insight into the results of the IPS or phrasing of questions from the screening questionnaire. For one employer who was unacquainted with IPS, the lack of confidence in the effectiveness of the IPS seemed to be prompted by the lack of knowledge about the implementation strategy of the IPS and ignorance about potential effects of the IPS.

Nearly all employees, regardless their level of acquaintance with the IPS, had confidence in the effectiveness of the IPS. Some employees acquainted with IPS mentioned this was due to their own positive experience with it. However, conflicting answers were given while some employees had less confidence in the strategy. Some expected that employees would not be honest about their health situation and others thought they would be honest. [Employee17] "Why do you need to conceal something? No, it is only to get better. Your health is important." According to many employees, the level of confidence in the strategy was determined to a large extent by the honesty of the employees when filling out the screening questionnaire.

Most employees regardless their level of acquaintance with the IPS, did not worry about discrimination or stigmatization related to the implementation of this IPS due to the 
perception that no personal information from the screening questionnaire would be shared with other employees. [Employee13] "If it works well nothing. Because nobody will know. I will know and the person who receives the answers from the Balansmeter will know. So if all goes well, that does not have any influence in the workplace.". They trusted that their risk score for LTSA would not be shared with colleagues and kept personal, so therefore discrimination would not be possible.

\section{Practical barriers related to the implementation}

Employers independent of acquaintance/unacquaintance with the IPS often indicated that finding time to fill out the screening questionnaire was considered a large barrier. Some employers acquainted with the IPS also mentioned the old-school look of the questionnaire and lack of an estimated completion time on the first page. A majority of the acquainted employers mentioned the low-frequency of the questionnaire as an issue. [Employer1] "It is a nice tool to measure something and then to do something with that measurement, but that measurement is only 1 time in 3 years so yes..." It only gave them a snapshot of the health state of their employees at one particular time, so they added more general interventions for the whole department. The use of emails as the only correspondence method was seen as a barrier by the unacquainted employers. They mentioned that communication about the IPS in general should be encouraged more.

The employees acquainted with the IPS often mentioned they were not able to fill in the screening questionnaire at work due to the time investment. [Employee13] "When we receive a questionnaire, it is impossible to say am going to stay behind my computer to fill in this questionnaire for the next hour". For the acquainted employees, the use of email was mentioned as a large barrier. They often did not have time to check their email regularly. "Usually I see it via e-mail, when I look at my own situation, when I return after a weekend, sometimes I have 200 e-mails" [Employee10]. Therefore, posters were mentioned as a tool to provide employees with the necessary information about the IPS. They also perceived the frequency of the screening questionnaire as a barrier, while their general state of health varied over time and could not be grasped once every three years. Some employees unacquainted with IPS mentioned the importance of company support for this strategy and one mentioned possible fear of discontent when care is only provided to certain individuals. A summary of the most important results is provided in Table 3. 
Table 3: Summary of the overall findings

\begin{tabular}{|c|c|c|c|}
\hline Themes & Sub-themes & Employers & Employees \\
\hline \multirow[t]{3}{*}{ Values } & Perceived responsibility for SA & $\begin{array}{l}\text { Large responsibility when related to work- } \\
\text { related/psychological complaints }\end{array}$ & $\begin{array}{l}\text { Shared responsibility depends on work- } \\
\text { related or non-work-related issues }\end{array}$ \\
\hline & Perceived responsibility for health & $\begin{array}{l}\text { Shared responsibility depends on work- } \\
\text { related or not work-related issues }\end{array}$ & $\begin{array}{l}\text { Shared responsibility depends on work- } \\
\text { related or not work-related issues }\end{array}$ \\
\hline & Presence of a health culture & Safety culture, focused on physical health & Safety culture, focused on physical health \\
\hline \multirow[t]{3}{*}{ Facilitators } & Effect of IPS on future SA & Awareness in employees' health & SA will decrease \\
\hline & Effect of IPS on future LTSA & $\begin{array}{l}\text { Majority positive but dependent on the } \\
\text { type of causes of SA }\end{array}$ & $\begin{array}{l}\text { Mixed feelings, dependent on the type of } \\
\text { cause of SA }\end{array}$ \\
\hline & Effect of IPS on health & Larger effect on psychological health & Positive about the effects on health \\
\hline \multirow{8}{*}{$\begin{array}{l}\text { Side effects \& } \\
\text { barriers }\end{array}$} & Costs (for this IPS) & Not considered an important factor & N/A \\
\hline & $\mathrm{ROI}$ & Only important if the costs are high & $\mathrm{N} / \mathrm{A}$ \\
\hline & Costs and benefit balance & Within normal ranges & $\mathrm{N} / \mathrm{A}$ \\
\hline & Healthcare costs & Increase in costs, followed by a decrease & $\mathrm{N} / \mathrm{A}$ \\
\hline & Healthcare use & Decrease, due to efficient use of healthcare & Decrease/increase, due to preventive actions \\
\hline & Costs (own payment for preventive care) & $\mathrm{N} / \mathrm{A}$ & Often seen as necessary \\
\hline & $\begin{array}{l}\text { Restraint own payment for use of } \\
\text { preventive care }\end{array}$ & $\mathrm{N} / \mathrm{A}$ & No restraint only if salary is insufficient \\
\hline & $\begin{array}{l}\text { Attitude towards employer if he pays for } \\
\text { preventive care }\end{array}$ & N/A & $\begin{array}{l}\text { Positive, some only when the health issue is } \\
\text { work-related }\end{array}$ \\
\hline \multirow[t]{3}{*}{$\begin{array}{l}\text { Adoption } \\
\text { barriers }\end{array}$} & Trust in the privacy of information & $\begin{array}{l}\text { Fear of employees not willing to share } \\
\text { personal information }\end{array}$ & $\begin{array}{l}\text { Fear of spreading personal information to the } \\
\text { employer }\end{array}$ \\
\hline & Confidence in the IPS & Confidence, with side notes & Confidence, when employees are honest \\
\hline & Discrimination/stigmatization & $\mathrm{N} / \mathrm{A}$ & No fear \\
\hline \multirow{3}{*}{$\begin{array}{l}\text { Practical } \\
\text { implementation } \\
\text { barriers }\end{array}$} & $\begin{array}{l}\text { Insufficient time to fill out the } \\
\text { questionnaire }\end{array}$ & Large barrier & Large barrier \\
\hline & Communication issues with the IPS & Issues due to the use of e-mails & Issues due to the use of e-mails \\
\hline & Low frequency of the IPS & Was perceived an issue & Was perceived an issue \\
\hline
\end{tabular}




\section{Discussion}

This study aimed to explore expected and perceived facilitators and barriers of an evidencebased indicated prevention strategy (IPS) for the reduction of future LTSA. We focused on employers and employees from large Dutch companies (acquainted and unacquainted with this strategy) and gained insight into the facilitators and barriers employing a qualitative study using semi-structured interviews with 20 employers and employees. The topic list was based on earlier research regarding worksite health preventive interventions and included a broad scope of topics and themes [18-27]. Purposive sampling was used to include respondents at different levels of an organization. Overall, the findings showed that, in general, the employers and employees had positive expectations concerning the effects of this IPS; in particular, the awareness provided by the screening questionnaire about the health situation of employees was appreciated. The ability of a preventive intervention to create awareness about the health situation was also mentioned by Goetzel et al.[30]. All employers and employees saw a potential benefit from the IPS for SA and psychological health. A large barrier according to the employers and employees was the fear of a privacy breach, the fear of spreading information about the health situation of employees to the employer. The explored facilitators and barriers showed to be related to personal or company values.

\section{Values}

The values as studied in the current study may be related to ethical dilemmas due to the dependent/unequal relationship between employer and employees [19]. Values like the perceived responsibility for SA and health may be involved with ethical issues, with the interests of the employer and employee needing to be balanced [31]. To determine whether something is perceived as a facilitator or barrier for this IPS, underlying values in an organization may be of great importance. This is in line with Beer et. al (1990) who stated that the organizational roles and the imposed responsibilities by the organization are very important in explaining the attitudes and beliefs of the individuals [32]. The health culture in an organization is presumed to be of great importance for the adoption of an intervention and perhaps also for its implementation [33]. While the underlying values are associated with the facilitators and barriers, these values could facilitate or hinder the adoption and implementation of the IPS under study. Our themes and subthemes related to expected facilitators and barriers of this IPS cannot, therefore, be seen as separate items that can be fixed on their own.

We found that employers and employees have in many cases different and opposing perceptions on the responsibility for SA and health. The employers feel primarily responsible when the SA or health impairment is related to work or psychological, and 
the employees feel responsible when their SA or their health is being affected by their life choices. These results are similar to the findings of Van Berkel [19], which also suggest that employers feel responsible for a healthy working environment and mental health aspects and the employees feel responsible for their lifestyle (and also perceive this as their autonomy). This division sounds logical, however, in reality, there is often no clear boundary between work-related and non-work-related SA or health complaints. It may be difficult to determine whether SA is work-related or not, particularly in the case of psychological or psychosocial health problems (which are multifactorial) [34]. This makes the responsibility for SA and health hazy for both the employers and employees and may hinder the adoption or implementation of preventive strategies.

The perceived responsibility for (types of) SA and health complaints seemed to be influenced by the health culture of an organization, especially for employees. The health culture was often aimed at improving physical health (e.g. eating healthy, enough exercise, safety) and less about psychological health improvement. The employees believed the employer was not responsible for SA or health related to psychological complaints, only safety at work. Possibly the health culture helped frame the way employees view their responsibility for $\mathrm{SA} /$ health and the responsibility for the employer. A qualitative study by Tonnon et al. 2014 showed similar results regarding the culture in the construction industry. Employees felt discouraged to openly discuss their health complaints and were afraid that other employees would see them as vulnerable [35]. Goetzel et al. [30] explained that when an organization lacks a supportive culture, health improvements that are sponsored by the employer are unlikely to give the desired results. Possibly, due to the lack of a health culture based on physical as well as psychological health, the employees do not feel supported with their psychological complaints and will therefore not comply with interventions to improve this or even create a blind spot.

To create support for this IPS and make its adoption and implementation easier, a more holistic health culture is expected to be beneficial. Therefore, the first steps could be to create more awareness of psychological health problems in the organization and encourage employees to disclose such complaints to the OP. Changing the health culture prior to implementation of the IPS seems not realistic. Nevertheless, employers should be informed/stimulated/made aware of the impact of a health culture on employee participation in health interventions such as this IPS.

\section{Facilitators and barriers}

Both employers and employees mentioned they had positive expectations about the effectiveness of this IPS, but the effect on future LTSA was not acknowledged by many employers. Preventing LTSA was perceived as something larger than this IPS, especially due to the low-frequency period of the strategy expected to be insufficient. Moreover, 
some employers also did not believe this strategy would be effective to prevent LTSA as, according to them, LTSA entails physical as well as psychological problems or solely physical problems, while the prevention of physical problems not being the focus of the IPS. Also some employees also associated LTSA more with physical complaints. Because the IPS was not considered useful for physical complaints, the overall efficacy related to LTSA was expected to be low. This is in contradiction with the scientific literature, showing that LTSA is most often related to psychological health complaints and would therefore be very appropriate [36, 37]. However, while the employees performed physical demanding tasks this could have resulted in more physical complaints than psychological complaints resulting in LTSA [38]. Nonetheless, there seems to be a strong association with psychological complaints and LTSA which may additionally to physical complaints be relevant for employees performing physically hard work. Therefore, less awareness about the LTSA risk factors might be regarded as a barrier for proper assessment of the impact of this intervention, and as such a barrier hindering implementation.

Another striking observed perception was related to employees' ideas about the concept behind the IPS. Many employees only mentioned the screening questionnaire and were unaware of the subsequent early consultation with the OHP for high-risk employees, followed by a health intervention, which is essential for the IPS to be effective. Employees often did not know the full content of the IPS, which may have resulted in an underestimation of its potential effectiveness. Even though the employees received all the information about the IPS from their companies, it is understandable that they forgot about steps 2 and 3 because only high-risk employees are invited for those steps and will only experience them when they agree to meet the OHP for an early consultation. Similar results were found in a comparable preventive study, also in an occupational health setting, albeit focusing on a different outcome (Cardio Vascular Disease (CVD)). Here, the employees were aware of the general goal of the intervention, decreasing the risk of a certain outcome, however, they were less informed about the approach of the intervention [35]. This lack of knowledge about the intervention may also be a barrier for implementation.

Often both employees and employers mentioned the fear of a privacy breach as a barrier for this IPS. However, in a similar study conducted amongst a similar group of employees regarding the elevated risk for CVD, no privacy fear was mentioned [35]. Possibly the factors explaining the elevated risk of CVD are often physical and therefore employees are less sensitive about the possibility that this information is leaked to the employer. A study on a digital mental health intervention at the workplace showed that stigmatization of mental illness is still a major problem [39]. As LTSA is often associated with psychological or mental issues, stigmatization and sensitivity of information may play a larger role in this context than in the context of CVD, and this may explain the importance of the fear of 
a privacy breach as an important barrier for the implementation of the IPS intervention. Some of our findings can be related to Fassier [21], where he investigated the barriers and facilitators of work disability prevention programs and combined his results in a framework. This framework includes complexity, needs, legislation, resources, organizational practices, professional practices, values, and benefits/risks [21]. A remarkable difference is the needs factor, a facilitator in the framework of Fassier [21], but more or less implicitly present in our findings. According to our results, the need for the employer to implement the IPS is clear, there is a need for the employers to save costs by reducing days of SA and occupational healthcare. The needs for the employees are less apparent, while the employees only receive a high or low risk score from the screening questionnaire, and only after the consultation with the OHP will hear how their scoring has been determined. Clear needs can only be tangible when people are aware of their current health situation. Therefore, the needs regarding this IPS are not yet visible for the employees because of its preventive nature, and employees do not see themselves already as at high risk to become chronically ill [28]. A high-risk score does not give the employees a clear overview about their current health state like e.g. the screening for lung cancer is able to. This is also in line with a coronary risk perception study, which showed that the understanding of the 'high-risk score' was mainly based on personal experience and often unrealistic and dichotomous [40]. Attaching meaning to the outcome 'high-risk' was perceived to be difficult also according to a Dutch construction worker study [27]. Therefore, the early consultation with the OHP will be crucial to address the meaning of the high-risk score and the associated health/personal issues. This can give the employees more clarity about their health status and their needs regarding the improvement of their health to prevent future LTSA.

\section{Strengths and limitations}

We took several efforts to increase the robustness of the results, including e.g. peer review and attaining saturation of the interview data. To increase rigor and validity a computer program was used to ensure a systematic analysis and audiotapes were used to provide objective recordings. The selection process of employers and employees was based on purposive sampling. The employees were first approached by a colleague from the occupational healthcare service, which they knew and possibly this created a sense of trust between the respondents and the interviewer. It is therefore likely this was fruitful for more accurate and rich information. However, the use of purposive sampling can lead to selective sampling and therefore might not provide an all-encompassing representation of the population. Nonetheless, in our opinion, this research included a wide sample variety in terms of acquaintance with the strategy, male/female employers, varying education levels from the employees, and the fact that saturation was reached. 
However, since the number of study participants was small (11 employees, 9 employers), we need to be cautious in interpreting these study results.

It is important to note that all employees in our sample were male, which is due to the large proportion of males in the participating organization. This may have influenced the feelings and perceptions about SA, health, and healthcare-seeking behavior. As shown by Thompson et al. [41], men are less likely to seek help for general care compared to women. A similar study with male construction workers also showed that men where less open in addressing their health issues compared to women [35]. Therefore, we need to be cautious in translating these findings to female employees.

Furthermore, studies have shown that a higher education is positively association with preventive behavior and lower education positively with non-seeking treatment [42, 43]. The education level of the respondents was not asked, but based on the job levels, we expect the education level of the employees to vary from low to high and we assume that most employers had high levels of education. As we do not have reliable information about educational level, the relation between education level and values, facilitators and barriers of this IPS could not be determined.

\section{Transferability of the results}

So far, only a few approaches to prevent LTSA, based on the principles of indicated prevention, are described in the literature [14, 15, 17]. As these trials have been conducted either in the Netherlands or in Finland, which has a similar social security system to the Netherlands, we might expect that the explored facilitators and barriers are applicable to a great extent to these or other indicated prevention strategies for SA. The IPS was implemented in organizations where the occupational service level is high compared to other organizations in the Netherlands. Therefore, we might assume that the trust in the occupational service under study was relatively high. We could hypothesize that for other organizations, with a lower level of occupational service, the aspect of trust in the occupational health service provider or health professionals might be even a greater barrier. Especially with regard to privacy-related issues, which rely strongly on trust, it can be an important barrier for employers and employees when the occupational service is considered to be less trustworthy. Additionally, the respondents were all working for large companies, and therefore it can be expected that some answers will be different for small medium-sized enterprises (SME). In particular, questions related to privacy may be seen differently if employers are less distant from their employees.

Although facilitators and barriers are likely to be intertwined with national legislation, in general, most countries in which the employer is responsible to pay for sick workers (such as northern European countries) have a high perceived incentive to implement a preventive strategy to prevent future LTSA. There is a gradient in employer responsibility 
for sick pay in European countries in terms of duration and liability (the difference between a few weeks to two years). A shift in a low or high incentive to decrease the risk to become chronically ill will depend on the period of sickness benefit. A short period will generate a high incentive for employees and a lower incentive for employers to prevent future SA. Implementing a preventive strategy will then be more important for employees. The applicability of our findings is of limited value for countries where there is almost no social security system, with regards to financial compensation of SA. This is because the responsibility for SA and the costs are shifted solely towards employees, which does not give the employers an incentive to implement a preventive strategy to prevent future LTSA.

\section{Recommendations for future research}

As far as we know, this was the first explorative study, investigating expected facilitators and barriers of an indicated prevention strategy for the prevention of LTSA. We used a semi-structured topic list which might have resulted in more narrow scope of answers. Although the topic list was carefully based on existing literature and other barriers have been consistently asked and explored. Future studies should consider using more openended questions to have a broad overview of all possible facilitators and barriers.

The educational level of our respondents was not taken into account in the current study, which may be of value for explaining the different views of respondents among themselves. For future research, it would be interesting to investigate this further. The respondents from the current study all worked in large organizations and therefore it would be interesting for future research to compare the results from this study with SMEs, to assess whether the same facilitators/barriers are visible or others appear.

\section{Recommendations for practice}

This study reveals important issues to improve the future adoption and implementation of this IPS. Educating the employers and employees regarding the true origin of LTSA and the three different steps of this IPS is an important first step towards better adoption and implementation of IPS. The employers and employees may then better understand the way this IPS works and how they could benefit from it and use this strategy to its full potential. Communication is a very important medium to address these apparent perceptions and should not only be done by the use of emails; posters can be a perfect medium for people who do not have time to read emails. Strategic communication, which entails education, motivation, market program offerings, and building trust is seen as one of the most important ways to maintain an effective intervention. The communication needs to be tailored and targeted so employers and employees of different age, sex, and education levels find the intervention appealing [44]. As this IPS focuses on high-risk 
employees who do not show clear signals of ill-health yet, the needs of these employees are not clearly visible for managers. It is therefore very important for an organization to use strategic communication and explicitly explain the purpose of this strategy to employees and employers alike.

To meet the concerns about privacy, the communication about this IPS needs to be transparent and embedded in the health culture of an organization. This might be a very difficult and long process, therefore it is probably more feasible to start with improving the lines of communication within an organization to provide all the information needed, concerning the privacy issues of employees regarding this IPS. To stimulate adoption, the health culture of an organization is very important and needs to include psychological as well as physical health, since it has an inevitable connection with LTSA [45]. This may provide an opportunity to openly discuss the feelings of responsibility for SA and health which can give more direction to the health needs of employees.

\section{Conclusion}

To conclude, all employers and employees indicated that they were positive about the effects of the IPS regarding SA in general. However, some important barriers were identified, mainly related to privacy issues and different observed discrepancies with regard to the IPS content and the true nature of risk factors associated with LTSA. To further facilitate the adoption and implementation of this IPS, these prevalent and often strong perceptions regarding the nature of LTSA and the content of this IPS need to be addressed, as the effectiveness of the approach will likely be underestimated and the preventive activities misunderstood. The fear of a privacy breach was mentioned as the most important barrier of this preventive strategy and deserves the utmost attention before and during implementation. 


\section{List of abbreviations}

LTSA= long-term sickness absence

$\mathrm{SA}=$ sickness absence

IPS= indicated prevention strategy

$\mathrm{OP}=$ Occupational Physician

$\mathrm{OHP}=$ Occupational Health Professional

\section{Declarations}

\section{Ethics approval and consent to participate}

The FHML REC committee of Maastricht University provided the ethical approval, the approval number is FHML-REC/2019/020. Informed consent was verbal and was asked from each respondent before the interviews started, this was approved by the ethics committee.

\section{Availability of data and materials}

The datasets generated and/or analyzed during the current study are not publicly available due to the personal and sensitive information from employers and employees. The data is only available on site after contact with the corresponding author on reasonable request, to ensure data access complies with the procedures of the General Data Protection Regulation (GDPR).

\section{Funding}

This study was financially supported by The Netherlands Organization for Health Research and Development (ZonMw), grant no 505312598027, by CAPHRI School for Public Health and Primary Care, Maastricht, The Netherlands. ZonMw has no role in any part of the research including the design, data collection, analysis, interpretation of the data, writing and reviewing.

\section{Acknowledgements}

We would like to thank Jos Slangen for his support in the data storage and handling and Michael van Boven for helping with the study logistics. 


\section{References}

1. Henderson M, Glozier N, Holland Elliott K. Long term sickness absence. BMJ. 2005;330(7495):802-3.

2. EU-OSHA. The Netherlands. EU-OSHA. 2019.

3. Rijksoverheid. Ziekteverzuim en herstel naar werk. 2019.

4. Rijksoverheid. Hoeveel loon krijg ik doorbetaald als ik ziek ben? Rijksoverheidnl. 2018.

5. van Amelsvoort LGPM, Jansen NWH, Kant I. Addressing long-term sickness absence: moving beyond disease, illness and work-related factors for effective prevention. Scand J Work Env Hea. 2017;43(1):1-4.

6. Vargas-Prada S, Demou E, Lalloo D, Avila-Palencia I, Sanati KA, Sampere M, et al. Effectiveness of very early workplace interventions to reduce sickness absence: a systematic review of the literature and meta-analysis. Scandinavian journal of work, environment \& health. 2016;42(4):261.

7. van Vilsteren M, van Oostrom SH, de Vet HC, Franche RL, Boot CR, Anema JR. Workplace interventions to prevent work disability in workers on sick leave. Cochrane Database of Systematic Reviews. 2015(10).

8. Vogel N, Schandelmaier S, Zumbrunn T, Ebrahim S, de Boer WE, Busse JW, et al. Returnto-work coordination programmes for improving return to work in workers on sick leave. Cochrane Database of Systematic Reviews. 2017(3).

9. Duijts SF, Kant I, Swaen GM, van den Brandt PA, Zeegers MP. A meta-analysis of observational studies identifies predictors of sickness absence. Journal of clinical epidemiology. 2007;60(11):1105-15.

10. Wikman A, Marklund S, Alexanderson K. Illness, disease, and sickness absence: an empirical test of differences between concepts of ill health. J Epidemiol Commun H. 2005;59(6):450-4.

11. Notenbomer A, Roelen C, Groothoff J, van Rhenen W, Bültmann U. Effect of an eHealth Intervention to Reduce Sickness Absence Frequency Among Employees With Frequent Sickness Absence: Randomized Controlled Trial. Journal of medical Internet research. 2018;20(10):e10821.

12. Lexis MA, Jansen NW, Huibers MJ, van Amelsvoort LG, Berkouwer A, Ton GTA, et al. Prevention of long-term sickness absence and major depression in high-risk employees: a randomised controlled trial. Occupational and environmental medicine. 2011;68(6):400-7.

13. (IOM) IoM. Classifications for Prevention. 2014.

14. Kant I, Jansen NWH, van Amelsvoort LGPM, Swaen GMH, van Leusden R, Berkouwer A. Screening questionnaire Balansmeter proved successful in predicting future long-term sickness absence in office workers. Journal of Clinical Epidemiology. 2009;62(4):408-14.

15. Taimela S, Malmivaara A, Justen S, Laara E, Sintonen H, Tiekso J, et al. The effectiveness of two occupational health intervention programmes in reducing sickness absence among 
employees at risk. Two randomised controlled trials. Occupational and Environmental Medicine. 2008;65(4):236-41.

16. Kant I, Jansen NWH, van Amelsvoort LGPM, van Leusden R, Berkouwer A. Structured early consultation with the occupational physician reduces sickness absence among office workers at high risk for long-term sickness absence: A randomized controlled trial. Journal of Occupational Rehabilitation. 2008;18(1):79-86.

17. Lexis MAS, Jansen NWH, van Amelsvoort LGPM, Huibers MJH, Berkouwer A, Ton GTA, et al. Prediction of Long-Term Sickness Absence Among Employees with Depressive Complaints. J Occup Rehabil. 2012;22(2):262-9.

18. Lexis MA, Jansen NW, Stevens FC, van Amelsvoort LG, Kant I. Experience of health complaints and help seeking behavior in employees screened for depressive complaints and risk of future sickness absence. Journal of occupational rehabilitation. 2010;20(4):537-46.

19. van Berkel J, Meershoek A, Janssens RM, Boot CR, Proper KI, van der Beek AJ. Ethical considerations of worksite health promotion: an exploration of stakeholders' views. BMC public health. 2014;14(1):458.

20. Allender S, Colquhoun D, Kelly P. Competing discourses of workplace health. Health: 2006;10(1):75-93.

21. Fassier JB. Identifying local obstacles and facilitators of implementation. Handbook of Work Disability 2013(Prevention and Management ):441-59.

22. Envicke B. Investing in a health workforce: the impact of physical wellness on psychological well-being and the critical implications for worker performance. Acad Health Care Manag J 2012;8;(1/2):21-32.

23. Gandy WM, Coberley C, Pope JE, Wells A, Rula EY. Comparing the contributions of well-being and disease status to employee productivity. Journal of occupational and environmental medicine. 2014;56(3):252-7.

24. Kivimaki M, Head J, Ferrie JE, Shipley MJ, Vahtera J, Marmot MG. Sickness absence as a global measure of health: evidence from mortality in the Whitehall II prospective cohort study. British Medical Journal. 2003;327(7411):364-8.

25. Hannon PA, Hammerback K, Garson G, Harris JR, Sopher CJ. Stakeholder perspectives on workplace health promotion: a qualitative study of midsized employers in low-wage industries. American Journal of Health Promotion. 2012;27(2):103-10.

26. Madison KM. The risks of using workplace wellness programs to foster a culture of health. Health Affairs. 2016;35(11):2068-74.

27. Damman OC, van der Beek AJ, Timmermans DR. Workers' knowledge and beliefs about cardiometabolic health risk. Journal of occupational and environmental medicine. 2014;56(1):92-100.

28. de Brouwer CPM, Verdonk P, van Amelsvoort LGPM, Jansen NWH, Kant I, Widdershoven GAM. Experiences of occupational physicians with the implementation of indicated prevention for 
long term sickness absence. Work. 2017;57(2):157-72.

29. Boeije H. Analysis in Qualitative Research SAGE2010. 209 p.

30. Goetzel RZ, Henke RM, Tabrizi M, Pelletier KR, Loeppke R, Ballard DW, et al. Do workplace health promotion (wellness) programs work? Journal of Occupational and Environmental Medicine. 2014;56(9):927-34.

31. North F, Syme SL, Feeney A, Head J, Shipley MJ, Marmot MG. Explaining Socioeconomic Differences in Sickness Absence - the Whitehall-li Study. British Medical Journal. 1993;306(6874):361-6.

32. Eisenstat R, Spector B, Beer M. Why change programs don't produce change. Harvard Business Review. 1990;68(6):158-66.

33. Randall C, Buys N, Kendall E. Developing an occupational rehabilitation system for workplace stress. International Journal of Disability Management. 2006;1(1):64-73.

34. Okpaku SO. Essential of Global Mental Health. 2014(ISBN 078-1-10702232-4):138.

35. Tonnon SC, Proper KI, van der Ploeg HP, Westerman MJ, Sijbesma E, van der Beek AJ. A qualitative study of the anticipated barriers and facilitators to the implementation of a lifestyle intervention in the dutch construction industry. BMC public health. 2014;14(1):1317.

36. Nielsen MB, Madsen IE, Bultmann U, Christensen U, Diderichsen F, Rugulies R. Predictors of return to work in employees sick-listed with mental health problems: findings from a longitudinal study. Eur J Public Health. 2011;21(6):806-11.

37. Dewa CS, Goering P, Lin E, Paterson M. Depression-related short-term disability in an employed population. J Occup Environ Med. 2002;44(7):628-33.

38. Carroll C, Rick J, Pilgrim H, Cameron J, Hillage J. Workplace involvement improves return to work rates among employees with back pain on long-term sick leave: a systematic review of the effectiveness and cost-effectiveness of interventions. Disability and rehabilitation. 2010;32(8):607-21.

39. Carolan S, de Visser RO. Employees' perspectives on the facilitators and barriers to engaging with digital mental health interventions in the workplace: qualitative study. JMIR mental health. 2018;5(1):e8.

40. van Steenkiste B, van der Weijden T, Timmermans D, Vaes J, Stoffers J, Grol R. Patients' ideas, fears and expectations of their coronary risk: barriers for primary prevention. Patient education and counseling. 2004;55(2):301-7.

41. Thompson AE, Anisimowicz Y, Miedema B, Hogg W, Wodchis WP, Aubrey-Bassler K. The influence of gender and other patient characteristics on health care-seeking behaviour: a QUALICOPC study. BMC family practice. 2016;17(1):38.

42. Bristow K, Patten S. Treatment-seeking rates and associated mediating factors among individuals with depression. The Canadian Journal of Psychiatry. 2002;47(7):660-5.

43. Gulliver A, Griffiths KM, Christensen H. Perceived barriers and facilitators to mental health help-seeking in young people: a systematic review. BMC psychiatry. 2010;10(1):113. 
44. Kent K, Goetzel RZ, Roemer EC, Prasad A, Freundlich N. Promoting healthy workplaces by building cultures of health and applying strategic communications. Journal of occupational and environmental medicine. 2016;58(2):114-22.

45. Nielsen MBD, Madsen IEH, Bultmann U, Christensen U, Diderichsen F, Rugulies R. Predictors of return to work in employees sick-listed with mental health problems: findings from a longitudinal study. European Journal of Public Health. 2011;21(6):806-11. 


\section{Additional file 1: Topic lists used for the semi-structured interviews}

\section{Interview topics for employers}

- Responsibility

o Responsibility for the health of the employee

- To what extent do you feel responsible for the health of the employee?

o Responsibility for sickness absence of the employee

- To what extent do you feel responsible for the sickness absence of the employee?

- To what extent do you, as an employer, think you can influence the sickness absence rate of your employees?

- Benefits of the preventive strategy

o Sickness absence

- What effect do you expect from this preventive strategy on the sickness absence of employees in your company?

- (If applicable) What effect have you seen from the preventive strategy on the sickness absence of employees in your company?

- (If applicable) Was this effect different from what was expected?

- Could the effect on sickness absence be different for different groups of employees?

o Health

- What is the meaning of the preventive strategy for different facets of employees' health?

- Healthcare use

- Do you expect more or less healthcare use?

- Do you perceive more or less use of healthcare as an obstacle for the preventive strategy?

- How and to what extent did you insure the risk for sickness absence of your employees?

- Culture

o Does health play a role in the culture of the company?

o How would you describe the health culture of the company?

- Barriers

o Trust the method

- To what extent do you have confidence in the preventive strategy?

- What do you think about the use of a preventive strategy to prevent longterm sickness absence? 
- Do feedback moments of the results from the preventive strategy contribute to an increase in trust in the preventive strategy?

- Barriers for the employees

- Do you expect barriers for your employees to participate in this preventive strategy?

o Costs

- To what extent do you have insight into the costs of the preventive strategy?

- How do you perceive the cost-benefit balance of this preventive strategy?

- How do you perceive high costs if the benefits are also high?

- To what extent does the Return On Investment (ROI) play a role in the implementation of the preventive strategy?

- If the financial benefits are for another person, how do you perceive the costs of this preventive strategy?

o Other

- Are there other barriers that can prevent the use of this preventive strategy?

\section{Interview topics for employees}

- Responsibility

o Responsibility for the health of the employee

- To what extent is the employer responsible for the health of the employee?

o Responsibility for sickness absence of the employee

- To what extent does the employer feel responsible for the sickness absence of employees?

- To what extent can the employer influence the sickness absence of an employee?

- Benefits of preventive strategy

o Sickness absence

- What effect do you expect from this preventive strategy on the sickness absence of employees in your company?

- Could the effect on sickness absence be different for different groups of employees?

o Health

- What is the meaning of the preventive strategy for different facets of employees' health?

o Healthcare use

- Do you expect more or less healthcare use?

- Culture

o Does health play a role in the culture of the company?

o How would you describe the health culture of the company? 
- Barriers

o Trust in the method

- To what extent do you have confidence in the preventive strategy?

- What do you think about the use of a preventive strategy to prevent long-term sickness absence?

- Do feedback moments of the results from the preventive strategy contribute to an increase in trust in the preventive strategy?

- Barriers for the employees

- Do you expect employees to face barriers to participate in this preventive strategy?

o Privacy

- What do you think about the privacy of the sensitive information that is requested in the questionnaire?

- In your opinion, is the privacy of the sensitive information sufficiently guaranteed?

o Discrimination

- To what extent do you believe that the use of the preventive strategy can lead to discrimination/stigmatization?

o Costs

- What do you think about paying for preventive care yourself?

- To what extent would your deductible prevent you from using preventive care?

- What will the employee think about the costs for preventive care for the employer?

- Are the costs for the employer an obstacle or an incentive to use preventive care?

o Other

- Are there other barriers that can prevent the use of this preventive strategy? 


\section{Additional file 2: Overview of the themes and codes from the interviews}

\section{Values}

Responsibility of employer for health of employee

Responsibility for health

Safety culture

Culture of change

Culture of fear

Responsibility of employee for health of employee

Responsibility for safety

Responsibility for sickness absence

\section{Facilitators}

Prevention

Personal attention

Coping with sickness absence

Interventions are different for sub-groups

General effect of preventive strategy

Modified work

Effect of IPS on health

Effect of preventive strategy for different sub-groups

Benefit of a preventive strategy

Stress related to work

Next steps following preventive strategy

Affecting behavior of employees

Need for valid measurements

Long-term sickness absence

Awareness given by the preventive strategy

Effect of IPS on sickness absence

Being a good employer

Office and factory difference

Different types of sickness absence

\section{Side effects \& barriers}

Private business

Uncertainty about preventive strategy

No trust in the manager

Incentive or impediment if employer pays healthcare costs

Barriers strategy

Barriers for employees 
More or less healthcare use as a result of this IPS

Deductible for preventive care

Financial benefits for someone else

Consequences of financial choices

High costs and benefits

Costs of preventive strategy

Costs and benefit balance

Costs after sickness absence

Medicalization

Need for correct positioning of the preventive strategy

Privacy of sensitive information

ROI

Discrimination or stigmatization by the use of this IPS

Trust in this IPS

Trust in the safety of sensitive information for employers and employees

Level of insurance for sickness absence

Withhold preventive care if deductible is needed

Employer pays preventive care

Healthcare use as a result of this IPS

Healthcare use differs by age 
Chapter 7

General discussion

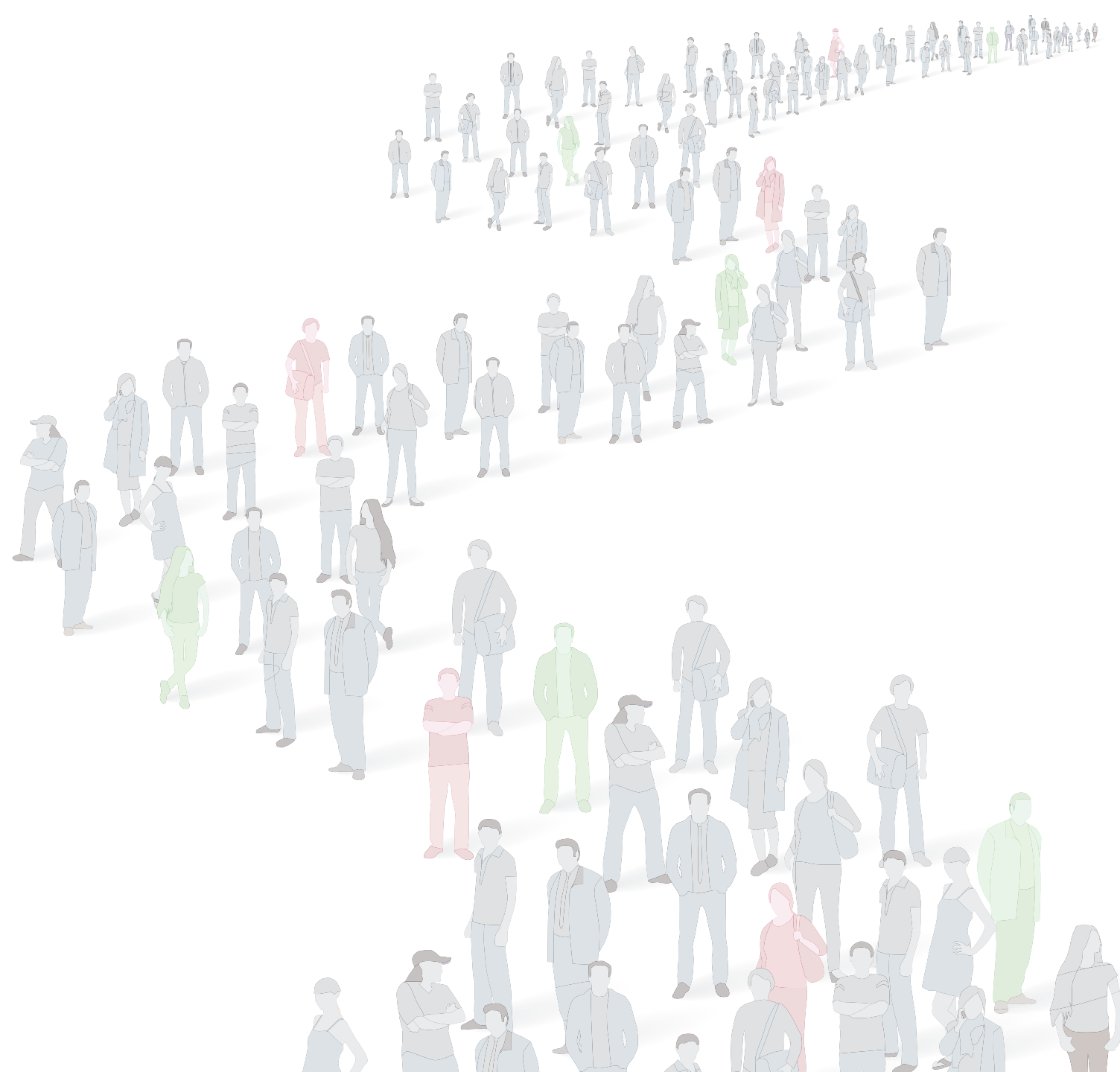





\section{General discussion}

An indicated prevention strategy (IPS) to prevent future long-term sickness absence (LTSA) was developed earlier. The strategy comprised a screening step in which employees were screened for being at high risk for future LTSA and the identified individuals at high risk, subsequently received an early intervention focused on the identified health/personal issue at stake [1, 2]. The short-term efficacy (1-year follow-up) was assessed for this IPS in a RCT (RCT I), which showed promising results, regarding the substantial decrease in days of sickness absence (SA) [2]. Furthermore, in a subsequent RCT, a tailored form of this strategy was assessed (RCT II), which aimed at employees at high risk for LTSA with additionally mild depressive complaints, receiving a protocolled psychological intervention, based on Problem Solving Therapy (PST) and Cognitive Behavioural Therapy (CBT) [3]. This tailored intervention showed in the RCT a large decrease in SA and a reduction in depressive complaints [3]. However, despite these promising results, the implementation of the IPS was lagging and therefore more research was necessary to substantiate the business case of such an approach, build on the principles of indicated prevention.

The overall aim of this thesis was to examine the IPS efficacy regarding sickness absence and health behaviour-related outcomes (health and well-being, social interactions, and healthcare usage) to enable a solid economic evaluation. A second aim was to evaluate the long-term efficacy of the IPS regarding sickness absence as well as the efficacy regarding ill health (diagnostic labels and attributions). Furthermore, this thesis aimed to investigate the facilitators and barriers for employers and employees regarding this IPS, which could benefit or hinder future implementation.

For this study, data from the previously described two RCT's regarding the efficacy of the IPS were used. Since the target population and the early intervention in both RCTs showed quite different results, we opted to analyse them separately and keep the study results separate.

In this general discussion, the main findings of the thesis are discussed and methodological considerations are described. Implications for different stakeholders are discussed, followed by recommendations for future research and the overall conclusion.

\section{Main findings}

To substantiate the business case of the IPS, it was important to investigate other outcomes of the IPS, apart from the reduction in days of SA and depressive complaints, which may be significant for different stakeholders. The efficacy of the IPS was assessed regarding health, well-being, social interactions, and healthcare usage during the 12 months of the first RCT. The results showed, against our initial expectations, only small improvements in well-being and social interactions for the intervention group. On the other hand, it was 
found that the intervention increased short-term healthcare usage, which was probably related to the preventive intervention. However, we could conclude, that the intervention did not lead to substantial medicalization. Therefore, the small improvement in wellbeing and social interaction, given the previously shown large decrease in days of SA, strengthened the business case of the IPS.

Since the effects of the IPS regarding health improvements and costs were known concerning healthcare usage and days of SA during the 12-months follow-up, it made an economic evaluation attainable, as seen from perspectives by different stakeholders (employer, employee, and society). The economic evaluation in this study was based on principles of a cost-effectiveness analysis (CEA), where the costs of SA solely occur as numerator and are not taking into account additionally as denominator, to prevent double-counting [4]. The economic evaluation may in the future be the basis of a sound comparison with other preventive interventions in public health, which is very important for relevant stakeholders.

The economic evaluation showed different outcomes depending on the different perspectives, with the employer perspective considering lower costs and greater effects, and the societal and employee perspectives considering greater effects and higher costs. Since the IPS involved multiple stakeholders, it was relevant to investigate the perspectives of the three most relevant stakeholders. The 7-12 months' time period showed lower costs and greater effects compared to the 0-6 months' period, indicating that the economic evaluation is likely to become more beneficial further over time. Especially given the decrease of SA which was maintained over a period of five years, as reported in chapter 4 . Chapters $2 \& 3$ focused on the short-term efficacy (12-months follow-up) of the IPS, but since the IPS was primarily developed to prevent future long-term sickness absence (LTSA), a long-term follow-up was imperative. The IPS as assessed in RCT I, showed a long-term decrease (5-year follow-up) in days of SA and LTSA, but this was not found for participants from RCT II, who received a psychological intervention based on CBT/PST. These large differences in long-term effects may indicate that a preventive intervention targeted at the identified health/personal problem at stake, may be more effective in preventing SA over 5-years follow-up compared to a psychological intervention. Moreover, employees from RCT II may have had a more complicated prognosis due to the additional depressive complaints, which might need a more specific treatment (involving different healthcare professionals), to establish a long-term decrease in SA and LTSA.

Apart from days of SA, also exit from employment was investigated during 5 years of follow-up and showed that employees who received the intervention from RCT II were more inclined to remain working for their employer compared to the control group. No difference regarding exit from employment was found for employees in RCT I. Also, in this case, the type of intervention may explain this difference, since the employees in RCT II 
received a psychological intervention that may have improved their coping abilities and made them more resilient and able to continue working. The long-term efficacy of the IPS regarding SA and exit from employment is very important for the business case of the IPS. Furthermore, these long-term effects are expected to generate higher economic benefits in terms of lower costs and greater effects compared to the current 12-months economic evaluation.

The previous chapters of this thesis all focused on the efficacy of the IPS in terms of SA and health behaviour-related outcomes. However, one might question whether the efficacy of the IPS is different for subgroups of employees with different spectra of factors and/ or complaints underlying the high risk status for future LTSA? To answer this question, days of SA were analysed according to their diagnostic labels and attributions (CAS \& CVO codes) during 5-years of follow-up. The IPS showed over a 5 years follow-up period, a statistically significant difference between the number of persons with different diagnostic labels (disease of the musculoskeletal system and somatic diseases) and attributes (workrelated and non-work related) for the intervention and control group. Moreover, there was a large difference in mean days of SA, classified as somatic during each year of the followup period. These results may contribute to a more tailored use of the IPS in terms of target population and time window.

The previous chapters all focused on the efficacy of this IPS. However, no matter how efficacious the IPS is, if employees and employers do not trust the strategy or do not cooperate, the strategy becomes useless. Therefore, exploring the facilitators and barriers for employers and employees regarding the IPS was necessary, especially to support much broader implementation. Overall, the respondents were positive about the benefits of the IPS; in particular, the awareness provided by the screening questionnaire about the health situation of the employees was acknowledged. One barrier often mentioned was the employees' fear of their health/personal information being forwarded to the employer. Barriers that may threaten future large-scale implementation were the observed discrepancies regarding the IPS content and the true nature of risk factors associated with LTSA. Furthermore, the company culture proved to be related to the explored facilitators and barriers. These outcomes demonstrated that employers should improve the internal communication about the IPS and safety measurements to increase the employees' compliance with the strategy and generate a higher economic benefit.

\section{Methodological strengths and weaknesses}

The specific strengths and weaknesses of the studies have been described in the discussion sections of chapters 2-6. Except for the qualitative study, the studies were all derived from a randomized controlled design, which is generally perceived as the most optimal one to examine the effect of a certain intervention compared to a care as usual situation. 
The data from chapter 2 (health behaviour-related outcomes) were derived from questionnaires completed by the intervention and control group during the 6 and 12 months of follow-up. Almost all health behaviour-related outcomes were based on validated questionnaires, which increased their comparability with other studies [5-11]. However, due to the 12 months follow-up period, it inhibited the assessment of possible long-term health behaviour-related outcomes. Since the current study only showed a small improvement in well-being and social interactions during 12 months of followup, the use of a longer follow-up seems better suited to assess the IPS efficacy regarding health behaviour-related outcomes.

The data for the economic evaluation (chapter 3) were similarly derived from the questionnaires at 6 and 12 months of follow-up. The economic evaluation was performed according to the CHEERS guidelines, which makes the results highly transparent $[12,13]$. Since in the current study the days of SA were only taken into account as costs and not additionally as effects, it ensures a proper methodological approach to calculate the costeffectiveness. As many occupational interventions used avoided days of SA as a health outcome, it inhibited a fair comparison. In the current thesis, effects were measured as self-perceived health since information regarding QALY's was not available from the questionnaires. This further complicated a fair comparison with other preventive interventions which used QALYs as effect measure.

Information regarding days of SA and exit from employment were available through company records and analysed over 5 years of follow-up. Data were available from all study participants in both RCTs, which ensured a valid and reliable method for the data collection. Disability pension records were seen as an important outcome of the strategy, since work disability involves high costs and may be derived from the inability to return to work after LTSA. However, unfortunately, due to privacy restrictions, we could only retrieve information regarding the exit from employment. Therefore, the reason behind the exit from employment, e.g. disability, retirement, job loss, or voluntary departure, could not be further investigated. Resulting in the omission of important components for the economic evaluation of the IPS, which could have resulted in a more positive outcome given the long-term decrease in LTSA.

The exact health status and reason for future sick leave were not known at the time of this early intervention. As a result, the impact of the IPS for different subgroups could only be investigated using diagnostic labels and attributes of SA spells after the preventive intervention, as a proxy for the ill health at the time of the intervention. SA labels and attributions were measured according to the Dutch Classification for Occupational Health Care and Social Affairs, in terms of diagnostic labels (CAS code) and attributions of SA periods (CVO code) [14]. The CAS and CVO codes were based on the ICD-10 classification, which makes them very comparable to other international studies. Moreover, for the 
attribution of work and non-work-related SA spells is the measurement as used in the current study, not expected to be very reliable, given the often diffuse origin of many health complaints. One cannot rule out that the answers from the screening instrument, which were available to the OP, could unconsciously, or consciously, have had an impact on the attribution of SA spells by the OP.

To explore the facilitators and barriers of the employers and employees, a qualitative design was considered optimal. The use of interviews, to ensure in-depth answers from the respondents related well with the choice of interview topics. However, due to the formerly arranged topic list, we might have directed the conversation. Possibly with more open-ended questions, other facilitators and barriers would have emerged.

During RCT I, 50.2\% of the employees responded to the screening questionnaire, and $38.2 \%$ during RCT II $[2,3]$. These screening uptake rates are far from preferred, however, normal and often seen in questionnaire studies and selective interventions. Nevertheless, improving the screening uptake is expected to result in large economic benefits, given the increased percentage of prevented LTSA. The same holds true for further optimizing the intervention compliance, which was in the study setting found to be around $66 \%$ for both RCTs [2, 3]. It is expected that our recommendations as discussed in chapter 5, when applied to enhance the implementation, can improve the response rates and compliance and as such, generate higher benefits in terms of health outcomes and costs.

\section{Implications for different stakeholders}

The IPS involved/may involve many different types of stakeholders (employee, employer, $\mathrm{OP} / \mathrm{OHP}$, OHS, insurance company, and society), each with their own benefits and costs. Nevertheless, since disability pensions could not be included in the current study, the implications for the insurance companies were disregarded. The business case of the strategy seen from different perspectives was a central focus of this thesis.

\section{Implications for the employee}

The small increase in well-being and social interactions shown in chapter 2 will generate benefits for the employees during the 12-months' of follow-up. The long-term decrease in SA and especially LTSA is expected to generate greater health benefits and simultaneously economic benefits. This may be especially valid for employees with an underlying somatic disease/illness as high risk determinant for future LTSA. The economic evaluation as performed in the current study (12-months follow-up) could not present the possible economic benefits for employees, since according to the Dutch national policy, the employer is responsible for all SA costs during the first year and often in the second year. As a result, the employee will only benefit economically after two years of SA, when $\mathrm{s} /$ he will receive a disability benefit, which is often much lower than their salary. Since 
the costs for disability benefits were not part of the current study, we may only speculate that the decrease in LTSA as described in chapter 4 will mirror a decrease in disability benefits and thereby generate a large economic benefit, which outweighs the costs for healthcare usage. Future studies should investigate the long-term economic evaluation, which may increase the uptake of and compliance with the IPS.

\section{Implications for the employer}

The IPS is comprehensive and employees need to be motivated to take up and comply with the strategy, which is time-consuming. Furthermore, the medical confidentiality of the consult with the OP/psychologist needs to be arranged, and the screening questionnaire sent to all employees. The relatively low uptake and compliance with the IPS, as is shown by previous publications is therefore concerning [2, 3]. The economic benefit of the IPS during 12-months' follow-up, as shown in chapter 3, is favourable for the employer, and given the greater effects and cost reduction during the 7-12 months' period compared to the 0-6 months, it is expected that the economic benefits will increase over time. Moreover, the long-term effects of the IPS regarding SA and the expected prevention of disability benefits makes the IPS even more engaging, given the specific Dutch situation in which the employer is responsible for paying up to 2 years of SA reimbursement costs and partly for the costs of disability pensions. The skewed distribution of costs in the first 2 years for Dutch employers and employees may be different in other countries with other social security systems e.g. the United States. Here, employees may be more inclined to participate, since the costs of SA are often at their own expense or at the health insurance company and not the expense of the employer. This different distribution of costs may stimulate the screening uptake and result in higher economic returns.

\section{Implications for occupational health professionals}

The proactive approach of the IPS demands a different working strategy from the OP/OHPs. They will play an essential role in making the high-risk status tangible to the employees and advise a fitting preventive intervention. Therefore, they received an extensive training to understand the IPS and another training which explains the International Classification of Functioning, Disability and Health (ICF), to make them better equipped to understand the high-risk status for LTSA of the employees and advise treatment and coping mechanisms. Since the uptake and compliance from employees are expected to be related to the conversation with the $\mathrm{OP} / \mathrm{OHP}$, it is probably useful to repeat the training once a year and include the OP/OHP more in the communication to the employees. 


\section{Implications for occupational health services}

The organization of the IPS is done by the occupational health service (OHS), with logistics that need arranging during the several steps of the strategy. To start with, the screening step involved mailing of the employees and gathering and processing of the screening questionnaires. The OHS identified the employees at high risk for future LTSA and invited them to a structured consultation with the OP/OHP. For employees from RCT II were the company counsellors specially selected to provide guidance with the preventive intervention. In general, the OHS had a more proactive approach to prevent LTSA before SA occurs. The greatest benefits in terms of fewer days of SA were seen for employees with a somatic diagnostic label, which may stimulate the OHS to focus more on the preventive interventions for these employees, given the large return on investment.

\section{Implications for society}

The number of employees responding to the screening questionnaire and following the preventive intervention was not high in either RCT. Therefore, only a small percentage of employees was detected as being at high risk for future LTSA and treated to prevent future LTSA. It should be noted that with regards to SA, only a small percentage of workers report periods of LTSA, however, these account for a major proportion of total SA days and SArelated costs. Since the current thesis has shown that LTSA has been prevented, one might assume that the intervention under study also indirectly prevents work disability and the shift of employees into disability pensions. Society is thus expected to benefit from more labour participation.

\section{Generalizability}

\section{Efficacy IPS}

The selection of study participants from the follow-up studies in chapters 2-5, were derived from the previously described RCTs. Therefore, the participants were all based on one population at the same company, which has consequences for the generalizability of the study results. The efficacy of the IPS was examined among workers in a large Dutch banking company from 2003-2008 for participants from RCT I and from 2007-2012 for participants from RCT II. It should be noted that SA behaviour changes over time, for example, because of the business cycle. Since the IPS from the first RCT is currently still in use and effective in the early detection of high-risk employees for LTSA, with a rather stable number of detected employees (source personal communication), one might assume that the results are not very dependent on the time of intervention.

The organization of the preventive strategy was performed by the OPs, and the structured 
early intervention was performed by the OP/OHP in RCT I and by certified psychologists during RCT II. The potential efficacy of the interventions, as studied by the RCTs, should be considered when extrapolating the results, to potential application in other settings and time windows. The uniform intervention group, involving one company, trained healthcare professionals, and the type and content of the intervention, all contributed to the high internal validity of the study. However, care should be taken when considering the efficacy of the interventions in other settings. Different outcomes may be expected when these factors change.

The outcomes regarding SA and LTSA are potentially more applicable to other large office companies since the questions from the screening questionnaire were specially selected for office workers. For companies with e.g. manual labourers or healthcare professionals, the screening questionnaire (Balansmeter) should involve other predicting questions to classify the high risk for LTSA. Different modules of the Balansmeter have already been developed for multiple types of work. Furthermore, the SA rate in a company is important for the ability to classify workers at high risk for future LTSA. The high-risk status for future sick leave was $6 \%$ in the company's population during RCT I and $2.4 \%$ (including additional mild depressive complaints) for the company's population during RCT II. Companies with a low LTSA rate might not benefit as much from the IPS, compared to companies with a higher LTSA rate, since the percentage of individuals at high risk for LTSA is expected to be lower, and thus fewer employees will receive a preventive intervention. It is assumed that, when a higher percentage of employees receives the intervention, the returns in terms of costs and health outcomes will be more beneficial.

The Dutch context should be taken into account for the transferability of the study results from all chapters. This is particularly true regarding SA, since it is often intertwined with the social or legal context within a country. This makes the results from the different chapters more applicable to countries with a comparable social system e.g. the northern European countries. Here the safety net for employees on sick leave is very high and the costs for SA are often covered by the employer or society. Therefore, the economic benefits for employees will be lower compared to countries where employees are not, or only partially, compensated for workdays lost due to SA. This, of course, could significantly change the economic evaluation of the IPS, as is stated in the implications for employers and employees.

\section{Exploring facilitators and barriers}

The uptake and compliance from employers and employees are critical for the efficacy of the IPS. Exploration of the facilitators and barriers was, therefore, crucial to understand the lag in the large-scale implementation of the strategy. Transferring the outcomes of the important facilitators and barriers that emerged from the data 
to medium-sized enterprises (SME) should be done with care since the answers were derived from employers and employees of large companies. In addition, the culture within a large company may be different compared to that of SMEs. Since the culture was very important for the facilitators and barriers, it is expected that a similar study with participants from SMEs might generate different results. Moreover, the facilitators and barriers study exposed important factors to improve the IPS uptake and compliance, which may have large consequences for the economic evaluation and result in greater health and economic benefits.

\section{Quality of the care as usual}

The occupational health service of the companies involved had a very high service level, which resulted in easy accessibility and high-quality occupational care by providing many different treatment options to both the intervention and control group. The control group of the RCTs received care as usual, which implied high-quality care provided by the occupational health service in case of SA or upon request. Therefore, in companies with a lower service level, there may be an increase in the contrast between the intervention and control group, resulting in greater effect sizes.

\section{Recommendations for future research}

Based on the results from this thesis, we recommend future research to strengthen and validate the outcomes of the different chapters and substantiate the business case of the IPS.

The current thesis showed a relation between the culture within a company and the facilitators and barriers of the IPS as mentioned by the employers and employees. Since the IPS efficacy was measured in a large office company, it is important to validate the study results in a company with a different culture.

Furthermore, as explained in earlier paragraphs of the discussion, the social context within a country is very important for the different perspectives of the economic evaluation. Since the current study only considers the Dutch context, it is recommended to investigate the efficacy of the IPS and economic evaluation in other countries with a different social context.

The OHS involved had a high service level and was therefore already easy accessible to employees. Therefore, for future research, the IPS efficacy needs to be studied in a company with a low OHS service level and investigate if the OHS service level is definable for the screening uptake and compliance.

Regarding health behaviour-related outcomes, only a small improvement in well-being and social interactions appeared during 12 months of follow-up. Given the large decrease in LTSA during 5-years follow-up, it is recommended for future studies to extend the 
follow-up period which may reveal higher health behaviour-related outcomes.

The economic evaluation of this thesis covered costs and effects during 12 months of follow-up. As explained earlier in the discussion, it is expected that an economic evaluation over a longer time period may generate lower costs and greater effects, but this needs to be validated in future studies. Moreover, the costs associated with disability pensions were not available in the current study due to privacy restrictions, but since they incur large societal costs, they should be included in future research. Furthermore, future studies should investigate the effect measure of an IPS in terms of Quality Adjusted Life Years (QALYs) or Productivity-Adjusted Life Years (PALYs) to incorporate all important health outcomes related to an indicated prevention in an occupational setting.

The frequency of the IPS, as currently implemented, involves a screening interval of once every three years and is based on an 'educated guess', which has not been validated by research. Future research should investigate the optimal screening period to detect highrisk individuals for LTSA and not hinder the screening uptake and compliance.

Furthermore, to improve the low screenings uptake and compliance, more research is needed to investigate the proper methods for communication, administration of questionnaires, and high-risk status communication, which are expected to improve the uptake and compliance.

The current study showed that for subgroups of employees with different spectra of factors and/or complaints, underlying the high risk for future LTSA, the results regarding the IPS efficacy in terms of SA were different and time depending. Future research should further disentangle the efficacy differences of this IPS and investigate the influence of the type of label and attribution on SA, to ensure higher economic and health benefits.

The exit from employment ensured interesting outcomes for participants from RCT II, which gives lead to investigating the efficacy of a CBT/PST intervention regarding exit from employment in terms of disability benefits, retirement, job loss, and voluntary departure. The IPS showed different results regarding participants from RCT I and II, which differed in study population and preventive intervention. More research is therefore needed to unravel the differences in IPS efficacy regarding participants with different health complaints and different types of preventive interventions. This may change the OP referral strategy and generate more savings.

\section{Conclusion}

Despite the recommendations for future research, this thesis showed that an indicated prevention strategy based on the prevention of future LTSA was able to decrease days of SA and LTSA over a long time period (5 years) for employees at high risk for future LTSA. This IPS showed a small improvement in well-being and social interaction during 12-months of follow-up. The economic evaluation showed an economic benefit as seen from the 
employer's perspective. Furthermore, the IPS showed a higher efficacy for employees with a somatic disease/illness label underlying their high-risk status for LTSA. Although some questions remain unanswered, the evaluation of the IPS showed a favourable overall outcome and therefore, indicated prevention may be considered an essential tool for the reduction of LTSA and to ensure benefits for occupational health and practice. 


\section{References}

1. Kant, I., et al., Screening questionnaire Balansmeter proved successful in predicting future long-term sickness absence in office workers. Journal of clinical epidemiology, 2009. 62(4): p. 408-414. e2.

2. Kant, I., et al., Structured early consultation with the occupational physician reduces sickness absence among office workers at high risk for long-term sickness absence: A randomized controlled trial. Journal of Occupational Rehabilitation, 2008. 18(1): p. 79-86.

3. Lexis, M.A., et al., Prevention of long-term sickness absence and major depression in highrisk employees: a randomised controlled trial. Occupational and environmental medicine, 2011. 68(6): p. 400-407.

4. Steenstra, I.A., et al., Economic evaluation of a multi-stage return to work program for workers on sick-leave due to low back pain. Journal of occupational rehabilitation, 2006. 16(4): p. 557-578.

5. Aaronson, N.K., et al., Translation, validation, and norming of the Dutch language version of the SF-36 Health Survey in community and chronic disease populations. Journal of clinical epidemiology, 1998. 51(11): p. 1055-1068.

6. Ware Jr, J.E. and C.D. Sherbourne, The MOS 36-item short-form health survey (SF-36): I. Conceptual framework and item selection. Medical care, 1992: p. 473-483.

7. DeSalvo, K.B., et al., Predicting mortality and healthcare utilization with a single question. Health services research, 2005. 40(4): p. 1234-1246.

8. Vercoulen, J.H., et al., Dimensional assessment of chronic fatigue syndrome. Journal of psychosomatic research, 1994. 38(5): p. 383-392.

9. Vercoulen, J., et al., Prognosis in chronic fatigue syndrome: a prospective study on the natural course. Journal of Neurology, Neurosurgery \& Psychiatry, 1996. 60(5): p. 489-494.

10. Goldberg, D.P. and V.F. Hillier, A scaled version of the General Health Questionnaire. Psychological medicine, 1979. 9(1): p. 139-145.

11. Karasek, R., The Job Content Questionnaire and User's Guide. Department of Industrial and System Engineering 1985.

12. Hakkaart-van Roijen, L., et al., Kostenhandleiding. Methodologie van kostenonderzoek en referentieprijzen voor economische evaluaties in de gezondheidszorg In opdracht van Zorginstituut Nederland Geactualiseerde versie, 2015.

13. Husereau, D., et al., Consolidated health economic evaluation reporting standards (CHEERS) statement. The European Journal of Health Economics, 2013. 14(3): p. 367-372.

14. Otto, W.C., Classification for Occupational and Insurance Medicine: Classification of complaints, diseases and causes for occupational and insurance physicians. [In Dutch: CAS: Classificaties voor Arbo en SV: Classificatie van klachten, ziekten en oorzaken voor bedrijfsen verzekeringsartsen]. 2002. 
Chapter 8

\section{Impact}

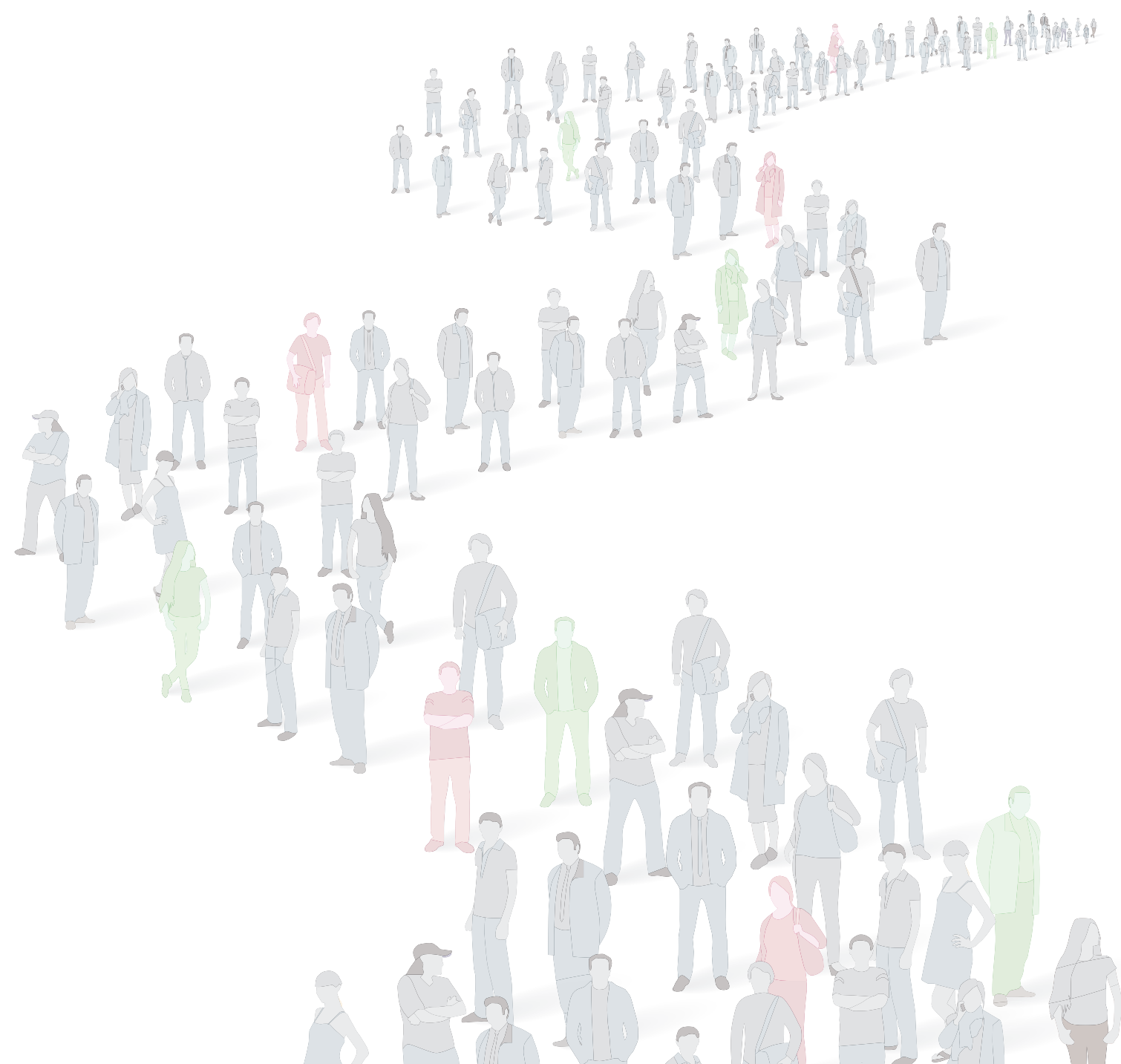





\section{Impact}

Long-term sickness absence (LTSA), which is defined as a period of 28 or more consecutive days of sickness absence (SA), often results in a large economic burden on the individual employee, employer, and the healthcare system, and comes with high societal costs overall [1-5]. The cost of continued payment of wages due to SA are estimated at 13.3 billion a year in the Netherlands [6]. Less than 10 percent of employees on sick leave are absent for more than 3 months in the Netherlands, however this group is responsible for $59 \%$ of all days of absence [6]. Especially employees who are absent for more than six months (4\% of the employees) are responsible for $37 \%$ of all days of absence [6]. Studies have shown, that LTSA has large consequences for the affected employees since it adversely affects well-being, mental and physical health and can lead to permanent work disability and early retirement due to ill health [2, 7-9].

The longer the duration of SA, the less likely it becomes that the employee will return to work [10]. Preventing LTSA might thus prove an important approach in addressing the issue. However, this might be challenging, given its multifactorial aetiology, resulting in a broad array of interrelated causal factors $[11,12]$. Since the origin of LTSA is often multifactorial, an indicated prevention strategy (IPS) might be very effective, as it is focused on a broad array of factors in contrast to preventive strategies based on the principles of general (primary) or selective prevention [11-14]. An IPS is focused on individuals within a population who are at high risk to achieve a specified negative outcome. Identifying these high-risk individuals requires a screening instrument, such as a screening questionnaire. Subsequently, these identified high-risk individuals are offered a preventive intervention [15].

This thesis is focused on an IPS that reduces the risk of future LTSA. The efficacy and impact over one year was already proven in two randomized controlled trials $[14,16]$. In this thesis, the long-term impact of the IPS (5-years) on sick leave as well as the impact of the IPS on the domains of health, wellbeing and healthcare usage were further investigated. Furthermore, the impact of the IPS was evaluated for different subgroups of diagnostic labels and attributes of SA spells as a proxy for the ill health of the employees at the time of the intervention. Moreover, a cost effectiveness analysis was performed as seen from the perspectives of the most relevant stakeholders.

From a valorization perspective, especially the long-term effects on SA, the cost effectiveness and participation with this IPS are important. Therefore, in this chapter, the knowledge gained from the studies, as described in this thesis, will be discussed in the light of the willingness of the workers to participate and the readiness of employers to implement this strategy. The long term effects and cost effectiveness of the strategy have already been covered in other chapters of this thesis. 


\section{Impact on uptake and compliance}

The effects of the study results from this thesis on multiple stakeholders have been discussed in various chapters of this thesis. However, since the screening uptake and compliance are two key elements of the (cost) effectiveness of the strategy, it is important to investigate if the new study results have changed the perception from the stakeholders regarding the usefulness of the strategy. A more positive view could potentially have a significant impact on the screening uptake and compliance of the strategy.

The uptake and compliance of the IPS is partly determined by the expected benefits from the strategy, which may be different for the different stakeholders. Employees may experience improvements related to health/well-being and possibly a reduction in SA/ healthcare costs later in time. Less SA cost, as a result of fewer days of SA will only affect employees after two years' time due to the social safety net of the Dutch policy. In the Netherlands, the employer bears the financial costs for sick employees and will pay their wages up until two years' time. After that period, the WIA (Work and Income according to Labour Capacity Act) will take over the wage payment, which reduces the payment to the employee. Therefore, the financial benefits of fewer days of SA will only be experienced by the employees after two years' time. However, for the employers the financial benefits from this IPS, as a result of fewer days of SA will be directly visible (e.g. less costs related to the wage payment for the employee on sick leave/replacement of the employee, occupational care etc.).

Furthermore, the employer decides if the IPS will be implemented in the company, however, the employees also decide for themselves if they are willing to fill in the screening questionnaire and are willing to comply with the preventive intervention. The willingness of the employees may also be determined by the information available regarding the strategy, the attitude of the employer, and the company culture (as explained in chapter 6). Therefore, the uptake and the compliance of this IPS is determined by many different factors, which should be taken into account when interpreting the possible effect of the new study results on the changed perception of the stakeholders regarding the usefulness of the strategy.

We contacted the employers, employees and occupational health professionals (OHP)/ health insurer, who were invited in the past for an interview regarding the IPS to answer similar questions with the use of an online survey. This approach enabled a proper assessment of the possible change in the perception of the IPS from the stakeholders given the new study results.

The questionnaire was send to $9 \mathrm{OHP} /$ health insurers, 23 employers and 9 employees. The questionnaire entailed questions related to the changed perceptions of the IPS in terms of the long-term efficacy related to SA and LTSA, health and well-being, healthcare usage and the economic evaluation. Followed by a question which captured the overall willingness 
to participate or implement this IPS, taking into account the new study results.

In total 41 questionnaires were send to the above mentioned stakeholders. This resulted in an overall response rate of $22 \%$, this was lower than expected, possibly due to the long time period (3 years) between the interview and the questionnaire. Some stakeholders had changed companies or were on pregnancy leave, which made them difficult to reach and less prone to return the questionnaire.

The new results of the IPS regarding the decrease in days of SA (chapter 4), were assessed by all respondents as reasonable to highly important for the positive change regarding their believe in the usefulness of the strategy. Moreover, the decrease in LTSA contributed similarly for all respondents in a positive change regarding their believe in the usefulness of the strategy. The results of the IPS regarding health and well-being (chapter 2) gave a more divergent answering pattern, from not useful at all to highly useful. With the highest percentage $(44.4 \%)$ of the respondents answering that the new information made a reasonable contribution to the expected usefulness of the strategy. These findings were comparable with the small effect sizes we found as explained in chapter 2. Similarly, for the results regarding healthcare usage the respondents answered divergent, here the largest number of respondents answering that it made a reasonable contribution to their perceived usefulness of the strategy (42.9\%). Furthermore, all employers and OHP/health insurers answered that the results regarding the economic evaluation were reasonable to highly important for a positive change in their perceived usefulness of the strategy.

Lastly, the respondents were asked overall, when taking into account the new IPS study results, if they would be more inclined to implement/participate with the IPS or saw more possibilities in daily practice. Most of the respondents (77.7\%) believed the new information regarding the IPS was considerable to highly important for future implementation/participation. Furthermore, only two respondents mentioned possible implementation barriers which were related to the lack of a good translation with their own company data and the lack of direct feedback from the screening questionnaire to decrease the time to the occupational healthcare service.

To summarize, given the new IPS results respecting SA, LTSA and the economic evaluation, the respondents were positive on the effects regarding the usefulness of the strategy. Although some caution is needed interpreting the results, given the rather low response rates. Overall, this may indicate that the new results may lead to an increase in uptake and compliance with the IPS and improve future implementation.

Improving the screening uptake and compliance is highly relevant given the relatively high costs of SA [6] on the one hand, and the screening costs on the other. Improving the screening uptake will hardly effect the costs for screening but is expected to result in less costs related to SA, which could give rise to an higher cost effectiveness. Given the current rather low implementation rate, the results as described in this thesis can 
help to increase the willingness of the workers to participate and increase the readiness of employers to implement this strategy. This expected positive change in uptake and compliance may improve the impact of the IPS on the employee, employer and society in terms of costs, participation and well-being. 


\section{References}

1. Taimela, S., et al., An occupational health intervention programme for workers at high risk for sickness absence. Cost effectiveness analysis based on a randomised controlled trial. Occupational and environmental medicine, 2008. 65(4): p. 242-248.

2. van Amelsvoort, L.G.P.M., N.W.H. Jansen, and I. Kant, Addressing long-term sickness absence: moving beyond disease, illness and work-related factors for effective prevention. Scandinavian Journal of Work Environment \& Health, 2017. 43(1): p. 1-4.

3. Labriola, M., Conceptual framework of sickness absence and return to work, focusing on both the individual and the contextual level. Work, 2008. 30(4): p. 377-387.

4. Lund, T., et al., Using administrative sickness absence data as a marker of future disability pension: the prospective DREAM study of Danish private sector employees. Occupational and Environmental Medicine, 2008. 65(1): p. 28-31.

5. Henderson, M., N. Glozier, and K.H. Elliott, Long term sickness absence. 2005, British Medical Journal Publishing Group.

6. Kraan K., I.d.M.M., de Vroome E., Health and safety balans; sickness abence and work incapacity. [In Dutch: Arbobalans 2020; ziekteverzuim en arbeidsongeschiktheid]. 2020. p. 111-127.

7. Helgesson, M., et al., Sickness absence at a young age and later sickness absence, disability pension, death, unemployment and income in native Swedes and immigrants. Eur J Public Health, 2015. 25(4): p. 688-92.

8. Waddell, G. and A.K. Burton, Is work good for your health and well-being? 2006: The Stationery Office.

9. Schuring, M., et al., The effect of ill health and socioeconomic status on labor force exit and reemployment: a prospective study with ten years follow-up in the Netherlands. Scandinavian journal of work, environment \& health, 2013: p. 134-143.

10. Henderson, M., et al., Work and common psychiatric disorders. Journal of the Royal Society of Medicine, 2011. 104(5): p. 198-207.

11. Schultz, I.Z. and R.J. Gatchel, Handbook of return to work: From research to practice. Vol. 1. 2015: Springer.

12. Alavinia, S.M., et al., Impact of work-related factors, lifestyle, and work ability on sickness absence among Dutch construction workers. Scandinavian journal of work, environment \& health, 2009: p. 325-333.

13. Kant, l., et al., Screening questionnaire Balansmeter proved successful in predicting future long-term sickness absence in office workers. Journal of clinical epidemiology, 2009. 62(4): p. 408-414. e2.

14. Kant, I., et al., Structured early consultation with the occupational physician reduces sickness absence among office workers at high risk for long-term sickness absence: A randomized 
controlled trial. Journal of Occupational Rehabilitation, 2008. 18(1): p. 79-86.

15. Mrazek, P., Haggerty, RJ, Reducing Risks for Mental Disorders: Frontiers for Prevention Intervention Research. 1994: National Academy Press: Washington, DC, US. 21.

16. Lexis, M.A., et al., Prevention of long-term sickness absence and major depression in highrisk employees: a randomised controlled trial. Occupational and environmental medicine, 2011. 68(6): p. 400-407. 


\section{Addendum}

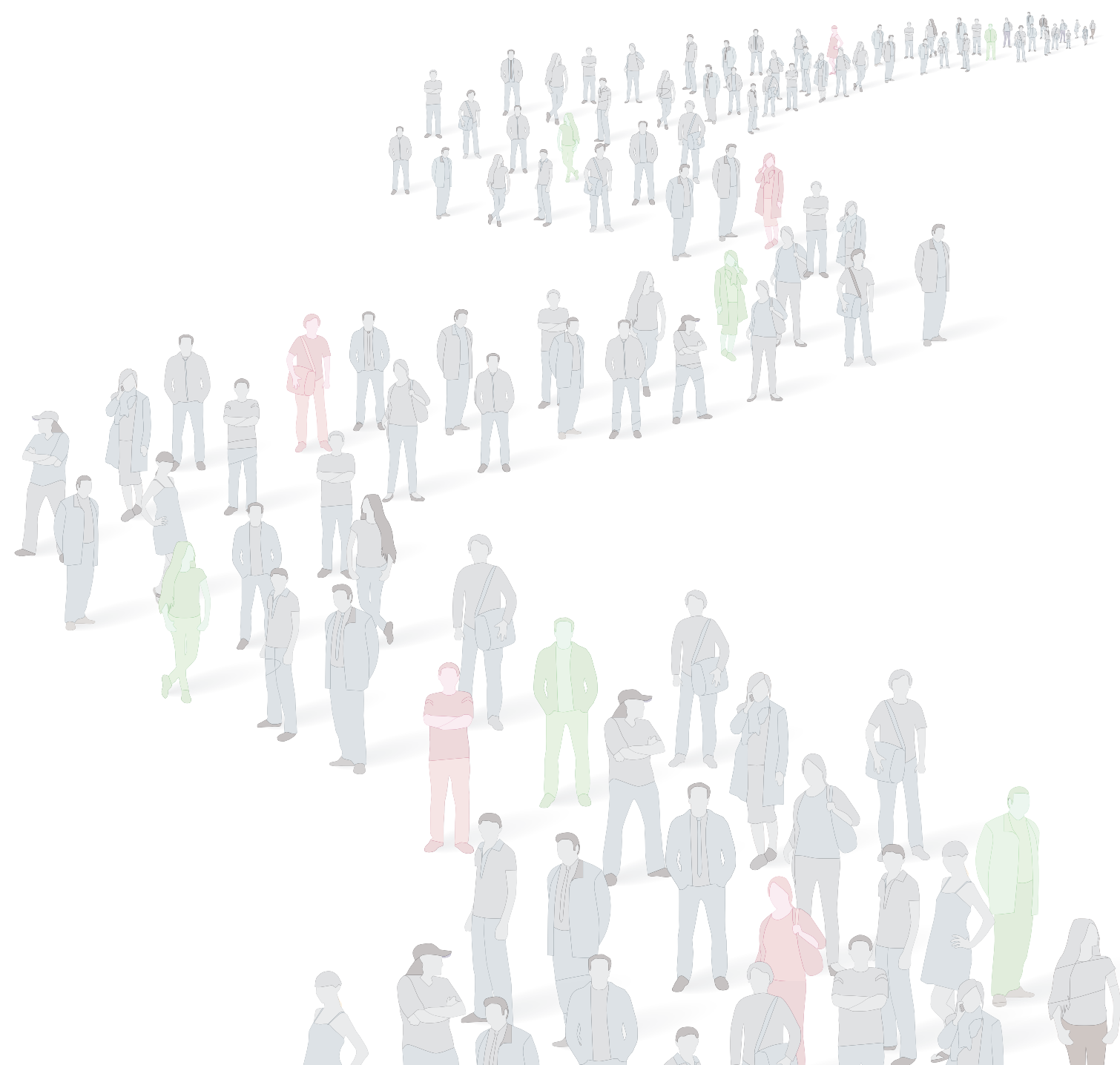





\section{Summary}

Long-term sickness absence (LTSA) is, and remains a large burden for developed countries and has considerable consequences for employees and employers and is accompanied by high societal costs. Although short periods of sickness absence (SA) are much more frequent than periods of LTSA (> 28 days), in total LTSA is responsible for $75 \%$ of the costs related to SA. Research has shown that reporting sick may negatively affect well-being, mental and physical health and could ultimately lead to permanent work disability and early retirement due to ill health.

The longer the duration of SA, it becomes less likely that an employee will return to work, possibly as well due to the increased detachment between the workplace and the worker over time. The prevention of LTSA is therefore very relevant. However, this has been proved challenging, especially given the multifactorial aetiology of LTSA with a broad array of interrelated causal factors. Return to work interventions (tertiary prevention) are often not highly effective, especially given the illness severity in this stage and the fact that the complaint/illness is often strongly intertwined with the work situation.

In contrast to population or general prevention, a preventive approach based on individual (indicated) prevention may result in better outcomes, since it is not restricted to one or two most important explanatory factors. Such an approach, based on the screening of employees at high risk for future LTSA, followed by a preventive intervention has been developed. This indicated prevention strategy (IPS) is based on a broad range of potentially interrelated factors. For this strategy, it is imperative to treat employees with starting health/personal complaints, who are at high risk for LTSA in the future, but are not yet on sick leave. The efficacy of this IPS regarding decreasing days of SA was already shown in two Dutch randomized controlled trials (RCTs), during a one-year follow-up. However, a large scale implementation of this IPS is lagging, possibly due to the lack of confidence of the stakeholders in the (cost) effectiveness of this strategy.

Therefore, the efficacy of this IPS is further investigated in this thesis, using extended follow-up data and additional data from the participants of these two RCTs. The first RCT (RCT I), focused on employees at high risk for future LTSA and the second RCT (RCT II), focused on employees at high risk for future LTSA with additionally mild depressive complaints. The preventive intervention from RCT I was specially focused on the identified health/personal issues at stake, while RCT II was a protocolised psychological intervention based on Cognitive Behavioural Therapy (CBT) and Problem Solving Therapy (PST). Since the target population and the early intervention in both RCTs showed quite different results, we opted to analyse them separately and keep the study results of the RCTs separate. Extended follow-up data, covering five years of follow-up 
was available for participants from both RCTs. Additional data was only available for participants from RCT I.

The primary objective of this study was to examine the IPS efficacy regarding a broad spectrum of explanatory outcomes, associated with health behaviour-related outcomes (health and well-being, social interactions, and healthcare usage) in order to assess the economic evaluation. The second objective was related to the long-term efficacy of this IPS regarding sickness absence. Furthermore, the efficacy of this IPS regarding diagnostic labels and attributions underlying sickness absence spells was researched. The last objective of this research aimed to investigate the facilitators and barriers of employers and employees regarding this IPS, which could benefit or hinder future implementation. To evaluate the efficacy of this IPS regarding health behaviour-related outcomes (health and well-being, social interactions and healthcare usage) data was analysed from RCT I during 12-monthts of follow-up (chapter 2 ). The results show only small improvements in well-being and social interactions for the intervention group. Furthermore, it was found that healthcare usage slightly increased in the short-term (6 months) for the intervention group, but the intervention did not result in substantial medicalization.

Chapter 3 presents the results of an economic evaluation of the IPS during 12 months of follow-up, as seen from a societal, healthcare and employer perspective. The economic evaluation showed divergent outcomes depending on the different perspectives. Using the employer perspective, the incremental cost effectiveness ratio (ICER) was dominant to the control with a cost saving of $€$-3.92 per point improvement of self-perceived health. Using a societal and healthcare perspective the IPS presented positive ICERs (€39.19 and $€ 39.77$ per point improvement in self-perceived health), indicating greater effects and higher costs for the intervention compared to the control group. The 7-12 months' time period showed lower costs and greater effects compared to the 0-6 months' period. This indicates that the economic evaluation over a longer time frame is likely to show a higher cost effectiveness as seen from all perspectives.

Earlier studies showed promising results regarding the efficacy of this IPS in decreasing days of SA, however, no studies were conducted regarding the efficacy beyond oneyear follow-up. Chapter 4 presents the efficacy of this IPS in terms of SA and exit from employment over five years of follow-up. For the high-risk employees classified by screening and receiving an intervention focused on their personal/health problems at stake, the IPS showed a long-term decrease in days of SA and LTSA during 5-years of follow-up. This long-term decrease in SA was however, not visible for the high risk employees with additional mild depressive complaints receiving the CBT/PST intervention. But in contrast, participants in the intervention group from this RCT were on average more inclined to remain working for their employer during 5 years of follow up, as compared to the control group. No difference regarding exit from employment 
was found for employees receiving the intervention focused on health/personal problems at stake.

The efficacy of the IPS was further examined by considering SA periods, with different spectra of factors and/or complaints underlying the high-risk status for future LTSA. The results of these analyses are described in chapter 5. SA labelled as somatic in the intervention group showed a statistically significant reduction in days of SA during 5 years of follow-up compared to the control group. Only during the first year, more days of SA were labelled as mental and behavioural disorders in the intervention group compared to the control group. Between 2-5 years of follow-up, statistically significant fewer days of SA were attributed as non-work-related in the intervention group compared to the control group and more days of SA attributed as work-related for the intervention group compared to the control group. Overall, the IPS efficacy was divergent for different spectra of factors and/or complaints of employees and dependent on the time window.

In chapter 6 the results are presented of a qualitative study regarding the facilitators and barriers regarding this IPS from employers and employees. Overall, the respondents were positive about the benefits of the IPS. In particular, the awareness provided by the screening questionnaire about the health situation of the employees was acknowledged. A barrier that was most often mentioned, was the fear that health and/or personal information would being forwarded to the employer. In addition, there were strong discrepancies in the risk factors associated with LTSA and the content of this IPS, which would result in an inhibition of the implementation. Furthermore, stakeholders should be aware of the impact a health culture may have on employee's participation regarding health interventions, such as this IPS.

In the general discussion (chapter 7), the main findings are presented and the internal validity of the separate studies and the generalizability of the results are discussed, as well as the implications of the findings from the perspectives of different stakeholders. We conclude that an indicated prevention strategy (IPS) focused on the prevention of future LTSA showed overall a favourable outcome and may be considered an essential tool for the reduction of LTSA. The economic evaluation of this strategy shows higher financial incentives for the employer than for the employee due to the Dutch social security system. For countries with another social security system the financial benefits are expected to be different. It is expected that this knowledge can/may increase the uptake and compliance from the stakeholders. 



\section{Nederlandstalige samenvatting}

Langdurig ziekteverzuim is en blijft een groot probleem in veel landen en gaat gepaard met ingrijpende gevolgen voor werknemers en werkgevers, en met hoge maatschappelijke kosten. Hoewel korte perioden van ziekteverzuim veel vaker voorkomen dan perioden van langdurig ziekteverzuim (> 28 dagen), is langdurig ziekteverzuim verantwoordelijk voor $75 \%$ van de verzuimkosten. Onderzoek heeft laten zien dat ziekteverzuim een negatief effect kan hebben op het welzijn, de mentale en fysieke gezondheid en uiteindelijk kan leiden tot blijvende arbeidsongeschiktheid en vervroegde uittreding als gevolg van een verslechterende gezondheid.

De kans dat een werknemer terugkeert naar het werk wordt kleiner naarmate de verzuimperiode langer aanhoudt, mogelijk ook door de in de loop van de tijd toenemende afstand tussen de werkplek en de werknemer. Het voorkomen van langdurig ziekteverzuim is daarom zeer relevant. Dit is echter een uitdaging, zeker gezien de multifactoriële etiologie van langdurig ziekteverzuim met een breed scala aan onderling gerelateerde causale factoren. Als verzuim eenmaal is opgetreden, zijn werkhervattingsinterventies (tertiaire preventie) vaak niet effectief. Dit komt voornamelijk door de ernst van de klachten en/of ziekte in een vergevorderd stadium en de vaak sterke verwevenheid van de gezondheidssituatie met de werksituatie die mogelijk de verandering in de gezondheid (gedeeltelijk) heeft veroorzaakt.

In tegenstelling tot populatie- of algemene preventie kan een preventieve aanpak op basis van individuele (geïndiceerde) preventie veel effectiever zijn, aangezien deze zich niet hoeft te beperken tot één of twee belangrijkste determinanten. Een dergelijke preventieve aanpak is ontwikkeld, gebaseerd op de identificatie (d.m.v. screening) van werknemers met een hoog risico op toekomstig langdurig ziekteverzuim, gevolgd door een preventieve interventie. Deze geïndiceerde preventieve strategie is gebaseerd op een breed scala aan mogelijk onderling samenhangende factoren. Voor deze strategie is het essentieel om werknemers te behandelen voordat ze met ziekteverlof zijn maar wel al beginnende gezondheids/persoonlijke klachten ervaren. Deze werknemers lopen een hoog risico op langdurig ziekteverzuim in de toekomst. Twee in Nederland uitgevoerde gerandomiseerde gecontroleerde onderzoeken (RCTs) hebben laten zien dat deze strategie effectief is in het verminderen van ziekteverzuim gedurende de looptijd van één jaar. Echter, de implementatie van deze strategie op grote schaal blijt achter, mogelijk door het gebrek aan vertrouwen van de stakeholders in de (kosten) effectiviteit van deze strategie.

In dit proefschrift wordt daarom verder onderzoek beschreven naar de werkzaamheid van deze strategie, met behulp van uitgebreide follow-up en aanvullende gegevens van de deelnemers aan deze twee RCTs. Het eerste gerandomiseerde onderzoek (RCT I) was gericht op werknemers met een hoog risico op toekomstig langdurig ziekteverzuim en het 
tweede gerandomiseerde onderzoek (RCT II) was gericht op werknemers met een hoog risico op toekomstig langdurig ziekteverzuim met daarnaast milde depressieve klachten. De preventieve interventie van RCT I was speciaal gericht op geïdentificeerde gezondheidsen/of persoonlijke problemen, terwijl RCT II was gericht op een geprotocolleerde psychologische interventie gebaseerd op cognitieve gedrags- en probleemoplossende therapie. Doordat de onderzoekspopulatie en de interventies in beide gerandomiseerde onderzoeken erg verschillende resultaten lieten zien, hebben we ervoor gekozen om deze apart te analyseren en de onderzoeksresultaten gescheiden te houden. Uitgebreide follow-up gegevens waren beschikbaar voor de deelnemers aan beide RCTs over een periode van 5 jaar. Aanvullende gegevens waren alleen beschikbaar voor deelnemers aan RCT I.

Deze studie had tot doel de werkzaamheid van een geïndiceerde preventieve strategie te onderzoeken m.b.t. een breed spectrum aan uitkomsten, gerelateerd aan gezondheidsgedrag (gezondheid en welzijn, sociale interacties en zorggebruik), teneinde een gedegen economische evaluatie mogelijk te maken. Een tweede doel was de lange termijn werkzaamheid van deze strategie met betrekking tot ziekteverzuim verder te onderzoeken. Verder had het onderzoek tot doel de werkzaamheid van de strategie te onderzoeken in termen van diagnostische labels en attributies die ten grondslag lagen aan het verzuim. De laatste doelstelling was het in kaart brengen van bevorderende en belemmerende factoren ten aanzien van deze strategie bij werkgevers en werknemers, die mogelijk de toekomstige implementatie kunnen bevorderen of tegenhouden.

Om de effectiviteit van deze strategie met betrekking tot uitkomsten gerelateerd aan gezondheidsgedrag (gezondheid en welzijn, sociale interacties en zorggebruik) te evalueren, werden gedurende 12 maanden gegevens geanalyseerd van deelnemers uit RCT I (hoofdstuk 2). De resultaten laten voor de interventiegroep slechts een kleine verbetering in welzijn en sociale interacties zien. Daarnaast zorgde de interventie voor een lichte toename in zorggebruik op de korte termijn (6 maanden), maar leidde de interventie niet tot substantiële medicalisering.

Hoofdstuk 3 presenteert de resultaten van een economische evaluatie van de strategie gedurende 12 maanden, gezien vanuit het perspectief van de maatschappij, de gezondheidszorg en de werkgever. De economische evaluatie liet uiteenlopende uitkomsten zien, afhankelijk van de verschillende perspectieven. Vanuit het werkgeversperspectief was de incrementele kosteneffectiviteitsratio (IKER) dominant met een kostenbesparing van $€-3,92$ per punt verbetering in ervaren gezondheid. Gezien vanuit het maatschappelijke en gezondheidsperspectief waren de IKERs positief ( $€$ 39,19 en $€ 39,77$ per punt verbetering in de ervaren gezondheid). Dit betekende een grotere effectiviteit en hogere kosten voor de interventiegroep ten opzichte van de controlegroep. Tussen de 7-12 maanden werden lagere kosten en grotere effecten gevonden in vergelijking met de periode tussen 
0-6 maanden. Dit geeft aan dat de economische evaluatie over een langere periode waarschijnlijk vanuit alle perspectieven een hogere kosteneffectiviteit zal laten zien.

Eerdere studies lieten veelbelovende resultaten zien van de werkzaamheid van deze strategie in het reduceren van verzuim. Er waren alleen nog geen resultaten bekend van studies die de werkzaamheid over een periode langer dan één jaar hebben onderzocht. Hoofdstuk 4 presenteert de resultaten van een langdurige studie (5 jaar) naar de werkzaamheid van deze geïndiceerde preventieve strategie in termen van verzuim en uitdiensttreding. Werknemers die door middel van screening werden geïdentificeerd met een hoog risico op langdurig ziekteverzuim en een interventie ontvingen specifiek gericht op persoonlijke- en/of gezondheidsproblemen, vertoonden een reductie in ziekteverzuim en langdurig ziekteverzuim over een observatieperiode van 5 jaar. Deze reductie van ziekteverzuim was echter niet zichtbaar binnen de groep werknemers met een hoog risico op langdurig verzuim die ook milde depressieve klachten rapporteerden en een interventie ontvingen gebaseerd op cognitieve gedrags- en probleemoplossende therapie. Wel werd voor deze werknemers (interventiegroep) opgemerkt dat ze gemiddeld veel langer werkzaam bleven bij hun werkgever in vergelijking met de controlegroep. Voor de werknemers uit RCT I die een interventie ontvingen gericht op gezondheids-/ persoonlijke problemen werd er geen verschil gevonden in de uitdiensttreding tussen de interventie en controlegroep.

De werkzaamheid van deze strategie werd verder onderzocht door de ziekteverzuim periodes te analyseren vanuit verschillende spectra van factoren en/of klachten die ten grondslag liggen aan het hoge risico voor toekomstig langdurig ziekteverzuim. De resultaten van deze analyses worden beschreven in hoofdstuk 5. Ziekteverzuim dat was gerelateerd aan somatische klachten in de interventiegroep, vertoonde statistisch significant minder dagen verzuim gedurende 5 jaar in vergelijking met de controlegroep. Alleen in het eerste jaar werden in de interventiegroep meer dagen ziekteverzuim gelabeld als een psychische en/of gedragsoorzaak, in vergelijking met de controlegroep. Tussen de 2-5 jaar na de interventie werden er statistisch significant minder dagen ziekteverzuim toegeschreven aan niet werk-gerelateerde factoren in de interventiegroep in vergelijking met de controlegroep. Tijdens dezelfde periode werden statistisch significant meer dagen ziekteverzuim toegeschreven aan werk-gerelateerde factoren in de interventiegroep vergeleken met de controlegroep. Samenvattend was de werkzaamheid van de strategie afhankelijk van het spectra aan factoren en/of klachten die ten grondslag lagen aan het ziekteverzuim en het tijdsvenster.

In hoofdstuk 6 worden de resultaten gepresenteerd van een kwalitatief onderzoek naar bevorderende en belemmerende factoren gerelateerd aan deze strategie, gezien vanuit de werkgevers en werknemers. Over het algemeen waren de respondenten positief 
over de voordelen van de strategie. In het bijzonder werd genoemd het positieve effect van de bewustwording van de gezondheidssituatie bij de werknemers. Een vaak genoemde belemmerende factor, was de angst van de werknemers voor het doorgeven van gezondheids- en/of persoonlijke informatie aan de werkgever. Daarnaast werden sterke discrepanties opgemerkt in de risicofactoren die verband houden met langdurig ziekteverzuim en de inhoud van de strategie. Deze discrepanties zouden mogelijk verdere implementatie kunnen remmen. Verder is het belangrijk dat de belanghebbenden zich bewust zijn van de gezondheidscultuur binnen de deelnemende bedrijven, gezien de invloed op het enthousiasme tot deelname van werknemers aan verschillende interventies op het gebied van gezondheid, zoals deze strategie.

In de algemene discussie (hoofdstuk 7) worden de belangrijkste bevindingen gepresenteerd en wordt de interne validiteit van de afzonderlijke onderzoeken en de generaliseerbaarheid van de resultaten besproken, evenals de implicaties van de bevindingen vanuit het perspectief van verschillende belanghebbenden. We concluderen dat een geïndiceerde preventieve strategie gericht op de preventie van toekomstig langdurig ziekteverzuim over het algemeen een gunstig resultaat heeft opgeleverd. De strategie kan worden beschouwd als een essentieel instrument voor het verminderen van langdurig ziekteverzuim. Door het Nederlandse socialezekerheidsstelsel laat de economische evaluatie van deze strategie voornamelijk financiële baten zien voor de werkgever en minder voor de werknemer. In landen met een ander sociaal systeem zullen de financiële baten mogelijk anders zijn. Naar verwachting kan/zal deze kennis de acceptatie en naleving van de strategie door de belanghebbenden doen verhogen. 



\section{Dankwoord}

Graag wil ik iedereen bedanken die een bijdrage heeft geleverd aan dit proefschrift. Allereerst wil ik de werknemers, werkgevers en Arbo professionals bedanken die hebben deelgenomen aan de interventies, interviews en/of de vragenlijsten. Zonder jullie bereidheid tot deelname had dit proefschrift niet kunnen bestaan. In het bijzonder wil ik nog een aantal mensen expliciet noemen.

Allereerst wil ik Ludovic van Amelsvoort, mijn copromotor, graag bedanken. Beste Ludo, jij was altijd beschikbaar voor mijn vragen, op kantoor kon ik altijd bij je binnenvallen en via Zoom was je ook altijd snel bereikbaar. Je begeleiding heb ik als bijzonder prettig ervaren, mede door de rust die je altijd goed in een project wist te bewaren. Ik heb veel van je geleerd en ben dankbaar voor de persoonlijke aandacht die jij altijd voor mij had. Vervolgens wil ik graag IJmert Kant, mijn promotor, bedanken. Beste IJmert, jouw positieve energie en heldere vertelwijze hebben mij destijds overtuigd om een promotietraject te beginnen binnen dit team. Tijdens mijn project wist je altijd goed het overzicht te bewaren en zorgde je er altijd voor dat alle neuzen dezelfde kant op stonden. Onze samenwerking, zeker in de laatste fase van dit project, heb ik als bijzonder prettig ervaren. Nicole Jansen, jouw constructieve feedback op de voortgang van de artikelen waren altijd erg waardevol. Bedankt ook voor je aanhoudende interesse in mijn onderzoek en privéleven. Jos Slangen, bedankt voor al je hulp bij het achterhalen van de juiste databestanden en het verwerken van de vragenlijsten. Inge Houkes bedankt voor alle hulp en constructieve feedback bij het kwalitatieve artikel en de leuke tijd in Denemarken. Silvia Evers, jouw directe manier van communiceren heb ik als bijzonder prettig ervaren. Bedankt voor al je kennis en hulp bij het artikel over de kosteneffectiviteit. Daarnaast wil ik ook graag Dave Stynen \& Bram Fleuren bedanken voor de fijne tijd op de afdeling.

Verder wil ik graag Gladys Tjin A Ton en Nikki Macintos van Arbodienst Beter, hartelijk danken voor de prettige samenwerking. Gladys, bedankt voor je feedback op de stukken en het snel beantwoorden van mijn vragen. Nikki, bedankt voor het tijdrovende uitzoekwerk naar alle verzuim- en gezondheidsdata van de participanten van de RCTs.

Jacqueline, ik vond het superleuk te horen dat jij een $\mathrm{PhD}$ ging doen binnen hetzelfde team en jij ook nog mijn kamergenoot werd. Samen konden we over alles praten en gelukkig ook heel goed zaken relativeren. Wat hebben we veel gelachen! Ik had me geen betere kamergenoot kunnen voorstellen. Heel veel succes nog met de laatste loodjes van je onderzoek, ik weet zeker dat het helemaal goed gaat komen! Pim, Jessica \& Karlijn, door jullie warme persoonlijkheden voelde ik mij al snel thuis op de afdeling. Jullie 
kamer werd al gauw een veilige haven waar iedereen zijn verhaal kwijt kon. Ik ben heel dankbaar voor jullie vriendschap en ben heel blij dat we elkaar nog steeds vaak spreken en zien. Pim \& Jessica, wat fijn dat jullie helemaal op jullie plek zitten qua werk, ik denk dat jullie functies heel goed passen bij jullie persoonlijkheid. Karlijn, ik ben heel trots op jouw doorzettingsvermogen en ik heb er alle vertrouwen in dat jij je eigen doelen gaat bereiken. Linda, Ariane, Tanja en Jacqueline, oftewel 'de dames van arbeid'. Ik wil jullie in het bijzonder bedanken voor alle steun en de prettige samenkomsten. Ik vind het mooi dat we elkaar nooit uit het oog zijn verloren, zelfs niet toen Linda een jaartje weg was van de afdeling. Daarnaast wil ik Tanja nog extra bedanken voor haar inzet en hulp. Jouw organisatietalent was tijdens de laatste fase van mijn PhD van grote waarde. Karlijn Hermans, hoewel je pas later bij ons op de afdeling bent gekomen konden wij het meteen al goed samen vinden. Je bent een pittige tante en dat vond ik meteen heel leuk. Ik vind het dan ook erg fijn dat we elkaar tijdens de coronatijd nog altijd zijn blijven zien. Veel wandelen, mountainbiken en natuurlijk heel veel 'echte' vrouwenpraat. Rachel, ook wel de 'gekleurde vogel met veren'. Je was een spraakmakende persoonlijkheid op de afdeling die wel van een goed feestje hield. Heel leuk dat we elkaar nog steeds zien en ook samen met onze mannen mountainbiken. Josien \& Kelly, jullie hebben de 'Amsterdammer' alles geleerd over het Limburgse feestvieren. Hierbij konden de kleurrijke schmink, gekke outfits en de Bifi-worstjes natuurlijk niet ontbreken. Ibrahim, the funny differences between the Dutch and Islamic culture, were often the topic of conversation. It was unfortunate your PhD journey did not last long, and you moved back to Saudi Arabia, but you always stayed in contact! Years later, you keep sending funny videos that always make me laugh. Alle AIO's wil ik daarnaast nog bedanken voor de gezellige lunches en vele vlaai momenten. Ik wens jullie allemaal nog veel succes met de afronding van jullie proefschriften.

Van de 'oudere garde' collega's wil ik graag nog twee mensen in het bijzonder bedanken; Adri \& Christel. Adri, door de jaren heen heb jij altijd veel interesse getoond in mijn onderzoek en in mij als persoon. Jouw gave om complexe zaken minder complex te maken en op humoristische wijze te kunnen relativeren, heb ik altijd heel fijn gevonden. Christel, met jou op de afdeling was het nooit saai! Altijd had je mooie verhalen te vertellen of had je weer iets geks meegemaakt. Ik heb altijd de passie bewonderd die je uitstraalt voor je werk en je studenten.

De dames van het secretariaat (Yvonne, Mariëlle, Petra en Ella), wil ik bedanken voor alle hulp en ondersteuning op de afdeling. Bij jullie kon ik altijd binnenlopen om iets te vragen of in te laten plannen. In het bijzonder nog een extra woord van dank voor Yvonne, die mij heeft geholpen bij het transcriberen van een deel van de interviews. Daarnaast wil ik graag Petra bedanken voor het inplannen van alle arbeidsafspraken en arbeidsuitjes. 
Michael van Boven, graag wil ik je bedanken voor de fijne samenwerking. Zonder jouw hulp hadden we nooit onze respondenten voor de interviews kunnen verzamelen. Heel fijn dat je altijd onze balansmeter bent blijven promoten!

Jos Bruystens, de mooie voorkant van het boekje en strakke lay-out heb ik aan jou te danken, veel dank daarvoor!

Lieve vrienden, ik mag blij zijn dat ik op veel plekken in Nederland heb mogen wonen en daardoor veel mooie mensen met een verschillende achtergrond heb leren kennen. Mijn Amsterdamse vriendinnen, oftewel 'Les filles', jullie staan altijd voor mij klaar en zijn zeker niet bang om jullie ongezouten mening te geven. Ik vind het leuk dat iedereen zijn eigen pad kiest maar we toch altijd tijd weten vrij te maken om elkaar te blijven zien. Mijn Rotterdamse, maar eigenlijk meer Finse vriendinnen (de Dutchies), zorgen altijd voor een lekkere borrel met veel vrouwenpraat. Heerlijk hoe we altijd kunnen praten over elkaars problemen en er vervolgens achter komen dat we niet de enige zijn met een bepaald probleem. Maria, wij kennen elkaar al vanaf ons zevende jaar en daarmee ben je mijn 'oudste' vriendin. Wij hoeven elkaar niet elke week te spreken, maar als we elkaar weer spreken of zien gaat het meteen als vanouds. Ik hoop jou en Woltjec heel snel in Warschau eindelijk te bezoeken! Lindsey Lindhoud, speciaal wil ik jou hier ook noemen. Onze tijd als huisgenoten in Rotterdam is alweer ver weg, maar gelukkig blijven we elkaar nog steeds opzoeken. Jij hebt altijd interesse getoond in mijn onderzoek en bent hier ook zeker een steun in geweest wanneer ik dit nodig had. Shannon \& Els, jullie geven altijd goed advies en ik geniet van onze leuke weekendjes in Rotterdam \& België. Bedankt Jister \& Alisa voor de leuke tijd in Maastricht! Als huisgenoten hebben we veel leuke en gekke tijden meegemaakt.

Lieve familie Klasen, ik geniet altijd ontzettend van onze feesten en verjaardagen. Jullie kunnen als geen ander plezier maken en weten altijd het mooie uit het leven te halen. Zeker in deze gekke tijden hebben jullie nogmaals laten zien wat voor harde werkers jullie zijn. Ik ben altijd trots op jullie. Lieve familie Starmans, hoewel wij ons nog niet officieel een familie mogen noemen voelt dit voor mij zeker wel zo. Jullie hebben mij opgenomen in een super lieve familie, die altijd voor elkaar klaarstaat. Bedankt ook voor de interesse die jullie steeds hebben gehad in mijn onderzoek.

Lieve mama \& Maarten, heel veel dank voor jullie onvoorwaardelijke steun. Waar ik ook woon of hoe laat ik ook bel, ik kan altijd bij jullie terecht. Bedankt ook voor het vertrouwen dat jullie altijd in mij hebben gehad. Maarten, mede door jouw overtuigingskracht ben ik jaren geleden deze PhD begonnen en is het toch maar mooi gelukt. 
Lieve Rik, jij hebt de gave om mij elke dag aan het lachen te maken en daarmee zorg je voor de nodige nuchterheid, die ik goed kon gebruiken tijdens dit traject. Ik heb heel veel bewondering voor de passie die jij als geen ander hebt voor jouw project, en je ongelofelijke doorzettingsvermogen. Ik ben blij dat wij elkaar vrijlaten om onze dromen na te jagen en ik ben heel benieuwd wat de toekomst ons gaat brengen. 


\section{About the author}

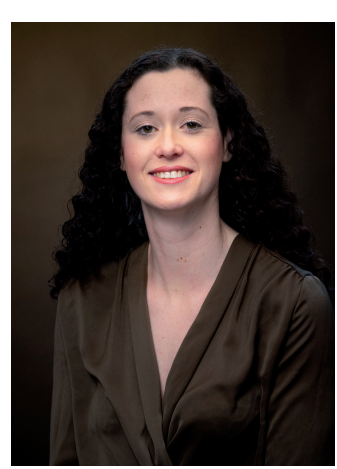

Sophie Klasen was born on December 8th, 1991 in Amsterdam, the Netherlands. After completing secondary school (VWO), she studied Health Sciences, Policy and Management at the Erasmus University in Rotterdam and obtained a Bachelor of Science degree in 2014. She acquired a Master of Science degree in Health, Economics, Policy and Law at the Erasmus University (Rotterdam, the Netherlands) and graduated in 2015, followed by a Master of Science degree in European Public Health at Maastricht University (Maastricht, the Netherlands). From January 2017 until July 2021 she worked as a PhD candidate at the department of Epidemiology at Maastricht University (Care and Public Health Research Institute, CAPHRI) under the supervision of Prof. IJmert Kant and Dr. Ludovic G.P.M. van Amelsvoort. Her PhD research project aimed to investigate the efficacy of an indicated prevention strategy focused on the prevention of future long-term sickness absence from an economic, health and stakeholders' perspective. The project was funded by the Netherlands Organization for Health Research and Development (Nederlandse organisatie voor gezondheidsonderzoek en zorginnovatie, ZonMw), and resulted in the present thesis. Sophie will continue her career as a research consultant at Déhora, focusing on workforce planning and management. 

